\title{
SELECTED GROUND-WATER-QUALITY DATA FOR THE WATER-RESOURCES INVESTIGATION OF MELLETTE AND TODD COUNTIES, SOUTH DAKOTA, 1990-96
}

By Janet M. Carter

U.S. GEOLOGICAL SURVEY

Open-File Report 97-248

Prepared in cooperation with the

SOUTH DAKOTA GEOLOGICAL SURVEY, the ROSEBUD SIOUX TRIBE, MELLETTE COUNTY, and the WEST RIVER WATER DEVELOPMENT DISTRICT

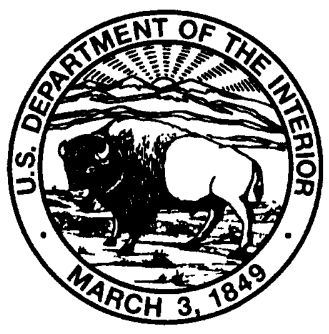




\title{
U.S. DEPARTMENT OF THE INTERIOR
}

BRUCE BABBITT, Secretary

\author{
U.S. GEOLOGICAL SURVEY
}

Gordon P. Eaton, Director

For addtional information write to:

Copies of this report can be purchased from:

District Chief

U.S. Geological Survey

1608 Mt. View Rd.

Rapid City, SD 57702

U.S. Geological Survey

Branch of Information Services

Box 25286

Denver, CO 80225-0046 


\section{CONTENTS}

Abstract
Introduction
Description of study area
Acknowledgments
Site identification
Quality-assurance program
Water-quality data
Reconnaissance water-quality data
Nitrate study water-quality data
Observation well water-quality data
Supplemental information

\section{ILLUSTRATIONS}

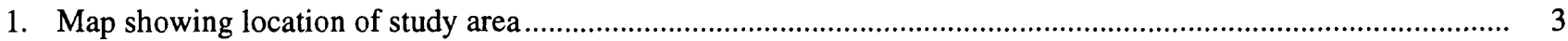

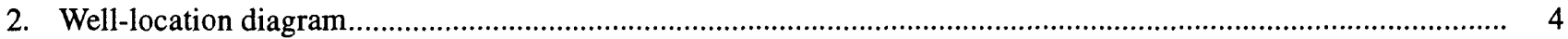

3. Map showing location of sites sampled during the reconnaissance water-quality program..................................... 7

4. Map showing location of sites sampled for the nitrate study ............................................................................. 8

5. Map showing location of sites sampled during the observation well water-quality program ................................. 10

\section{TABLES}

1. Physical properties and concentrations of inorganic constituents from reconnaissance sampling of alluvial aquifers

2. Physical properties and concentrations of inorganic and organic constituents from reconnaissance sampling of the Ogallala aquifer

3. Physical properties and concentrations of inorganic and organic constituents from reconnaissance sampling of the Arikaree aquifer

4. Physical properties and concentrations of inorganic constituents from reconnaissance sampling of the White River aquifer

5. Physical properties and concentrations of inorganic and organic constituents from reconnaissance sampling of the Pierre Shale aquifer .

6. Physical properties and concentrations of inorganic constituents from reconnaissance sampling of the Dakota aquifer

7. Physical properties and concentrations of inorganic constituents from reconnaissance sampling of unidentified aquifers

8. Quality-assurance results of field blank sample collected during the reconnaissance sampling program

9. Chemical analyses of field duplicates for reconnaissance water samples

10. Precision of chemical analyses of field duplicates for reconnaissance water samples

11. Selected water-quality data from observation wells and private wells completed in the Ogallala aquifer sampled by the U.S. Geological Survey for the nitrate studies

12. Quality-assurance results of blank sample collected during the nitrate study

13. Chemical analyses of field duplicates for nitrate study water samples

14. Selected water-quality data from observation wells completed in alluvial aquifers sampled by the U.S. Geological Survey...

15. Selected water-quality data from observation wells completed in the Ogallala aquifer sampled by the U.S. Geological Survey 


\section{TABLES-Continued}

16. Selected water-quality data from observation wells completed in the Arikaree aquifer sampled by the U.S. Geological Survey

17. Selected water-quality data from observation wells completed in the White River aquifer sampled by the U.S. Geological Survey

18. Quality-assurance results of blank samples collected during the observation well sampling program ... 72

19. Chemical analyses of field duplicates for observation well water samples

20. Precision of chemical analyses of field duplicates for observation well water samples

CONVERSION FACTORS

\begin{tabular}{rcl}
\hline Multiply & By & To Obtain \\
\hline acre & 4,047 & square meter \\
acre & 0.4047 & hectares \\
feet & 0.3048 & meter \\
inch & 25.4 & millimeter \\
square miles & 2.590 & square kilometer \\
\hline
\end{tabular}




\title{
Selected Ground-Water-Quality Data for the Water-Resources Investigation of Mellette and Todd Counties, South Dakota, 1990-96
}

\author{
By Janet M. Carter
}

\section{ABSTRACT}

This report presents data on water quality that have been collected from 1990 through 1996 for the Water Resources Investigation of Mellette and Todd Counties, South Dakota. The investigation is a cooperative effort between the U.S. Geological Survey, the South Dakota Geological Survey, the Rosebud Sioux Tribe, Mellette County, and the West River Water Development District.

A total of 100 samples were collected by the U.S. Geological Survey and the Rosebud Sioux Tribe for a reconnaissance ground-water sampling program. During this sampling program, private domestic wells in Todd and Mellette Counties were sampled to determine general water-quality properties of the aquifers and to determine if any potential water-quality problems existed. All of the reconnaissance samples were analyzed for field measurements, common ions, and nitrates. Some of the samples were analyzed for trace elements, nutrients, and organic compounds.

During the reconnaissance sampling, an area in south-central Todd County was identified as having high nitrate concentrations. The U.S. Geological Survey investigated nitrate concentrations in south-central Todd County in independent studies during 1990-91 and 1994. These data have not been published previously and are presented in this report.

A comprehensive drilling program was initiated in cooperation with the South Dakota Geological Survey in 1992. Seventy-eight test holes were drilled and 56 observation wells were installed between 1992 and 1996. A total of 30 water-quality samples were collected from selected observation wells in 1994, and a total of 17 were collected in 1996 . These samples were analyzed for field measurements, common ions, nutrients, trace elements, and radiochemicals.

This report presents the water-quality data that were collected during the reconnaissance, nitrate, and observation-well sampling programs. Quality-assurance data collected during the three sampling programs also are presented.

\section{INTRODUCTION}

The Water Resources Investigation of Todd and Mellette Counties, South Dakota, was initiated in 1992 as a cooperative effort between the U.S. Geological Survey (USGS), the South Dakota Geological Survey (SDGS), the Rosebud Sioux Tribe, Mellette County, and the West River Water Development District.

Prior to the water-resources investigation, a reconnaissance ground-water sampling program was accomplished during 1990 by the USGS and the Rosebud Sioux Tribe. The purpose of this sampling program was to sample private domestic wells in Todd and Mellette Counties to determine general water-quality properties of the aquifers and to determine if any potential water-quality problems existed. All of the reconnaissance samples were analyzed for field measurements, common ions, and nitrates. Some of the samples were analyzed for trace elements, nutrients, and organic compounds. A total of 100 samples were collected.

During 1990, the SDGS investigated nitrate concentrations in parts of Todd and Mellette Counties (Hammond, 1994). The USGS also investigated nitrate 
concentrations in south-central Todd County in independent studies during 1990-91 and 1994 that were accomplished in cooperation with the Rosebud Sioux Tribe and the SDGS. These data have not been published previously and are presented in this report.

After the water-resources investigation started in 1992, a comprehensive drilling program was initiated in cooperation with the SDGS. Seventy-eight test holes were drilled and 56 observation wells were installed between 1992 and 1996. Water-quality samples were collected during 1994 and 1996 from selected observation wells. These samples were analyzed for field measurements, common ions, nutrients, trace elements, and radiochemicals. A total of 30 samples were collected during 1994 and a total of 17 were collected during 1996.

This report presents the water-quality data that were collected during the reconnaissance, nitrate, and observation-well sampling programs. Quality-assurance data collected during the three sampling programs also are presented.

\section{Description of Study Area}

Todd and Mellette Counties are located in southcentral South Dakota (fig. 1). Todd County has an area of 1,388 square miles and Mellette County has an area of 1,306 square miles. The original boundaries of the Rosebud Indian Reservation included all or nearly all of Gregory (located east of Tripp County), Mellette, Todd, and Tripp Counties, and a small portion of Lyman County. In the early 1900's, the Rosebud Reservation was opened up for homesteading. As a result, scattered tracts of non-Indian land are present in both Todd and Mellette Counties. In 1975, the Rosebud Indian Reservation boundary was revised to include only Todd County.

Although the drilling was concentrated in Todd and Mellette Counties, a few of the wells were drilled in Bennett County just outside the western boundary of Todd County, in Jones County just outside the northern boundary of Mellette County, and in Tripp County just outside the eastern boundary of Todd County. The wells that were sampled for this study are completed in the aquifers of the following formations listed from oldest to youngest: Dakota Sandstone (Cretaceous age), Pierre Shale (Upper Cretaceous age), White River Group (Oligocene age), Arikaree Formation (Miocene age), Ogallala Formation (Pliocene age), and undifferentiated alluvial deposits (Pleistocene and Holocene age). Detailed descriptions of the formations and a map showing the location of the surficial deposits in Todd and Mellette Counties are presented in Ellis and others (1971).

\section{Acknowledgments}

Many people have assisted with development and implementation of the water-resources investigation. In particular, Syed Huq, Charles Mack, and John Whiting of the Office of Water Resources for the Rosebud Sioux Tribe and Sena Lauritsen of the Mellette/ Todd Water Resources Coordination Project provided valuable assistance including the collection of waterquality samples. The SDGS drilled the test holes and installed the observation wells. Dick Hammond and Patricia Hammond of the SDGS provided valuable insight and technical guidance. The author also acknowledges the cooperation of residents of Mellette and Todd Counties for providing information concerning their private wells.

\section{SITE IDENTIFICATION}

Three methods of station identification are presented in the water-quality tables in the Supplemental Information section at the end of the report. The first method is the station identification number, which is based on the international system of latitude and longitude. The number contains 15 digits. The first six digits denote the degrees, minutes, and seconds of latitude north of the equator. The next seven digits denote the degrees, minutes, and seconds of longitude west of the prime (Greenwich) meridian. The last two digits are sequential numbers for wells within the same latitude and longitude.

The second identification method is the local number, which is based on the Federal land-survey system of western South Dakota (fig. 2). The local number consists of the township number followed by "N," range number followed by "W," and the section number followed by a maximum of four uppercase letters that indicate, respectively, the 160-, 40-, 10-, and 2.5acre tract in which the well is located. These letters are assigned in a counterclockwise direction beginning with " $A$ " in the northeast quarter. A serial number following the last letter is used to distinguish between wells in the same 2.5-acre tract. Thus, well 38 N28W36ABCB is in the NW1/4 of the SW1/4 of the NW1/4 of the NE1/4 of section 36 in township 38 north and range 28 west. 


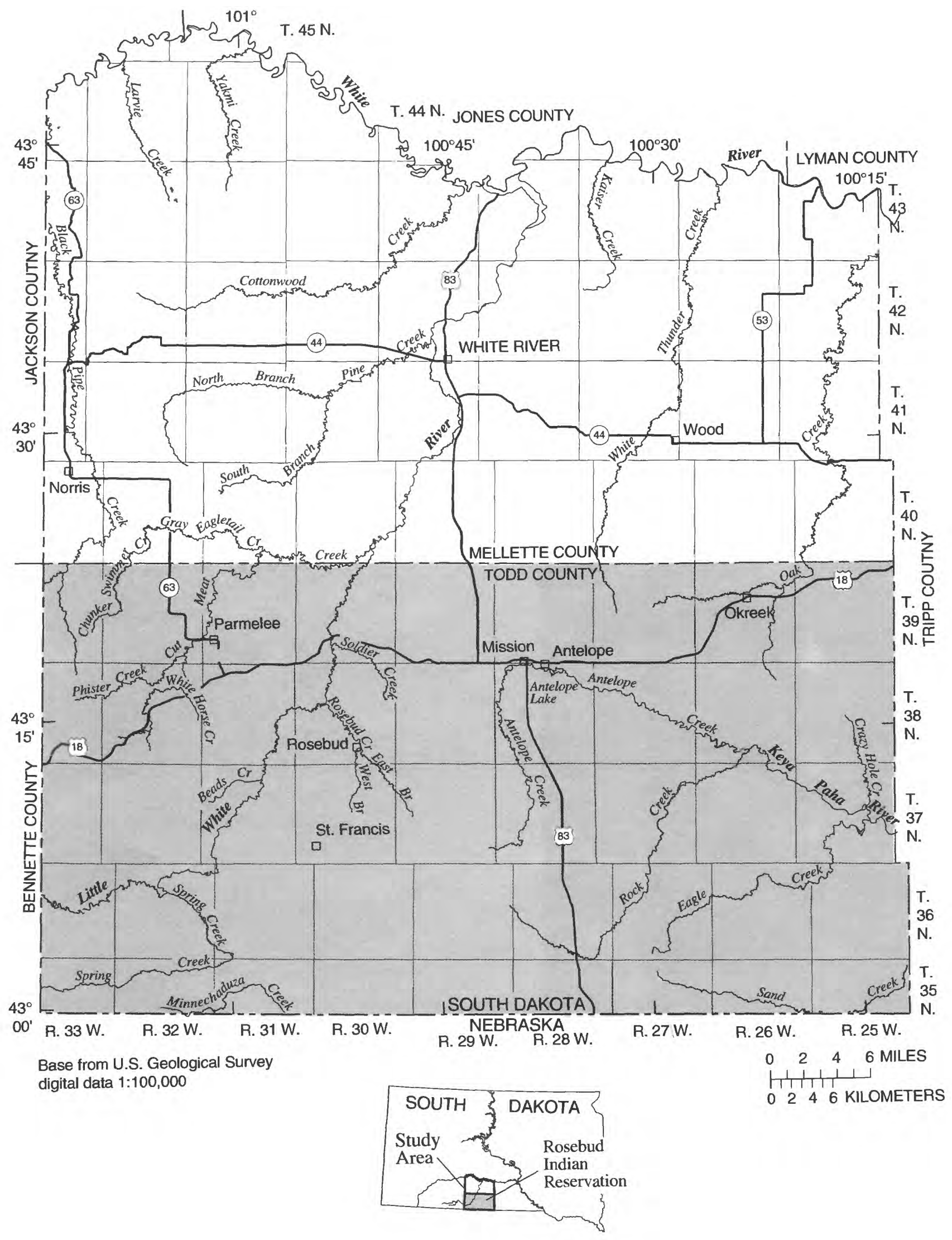

Figure 1. Location of study area. 


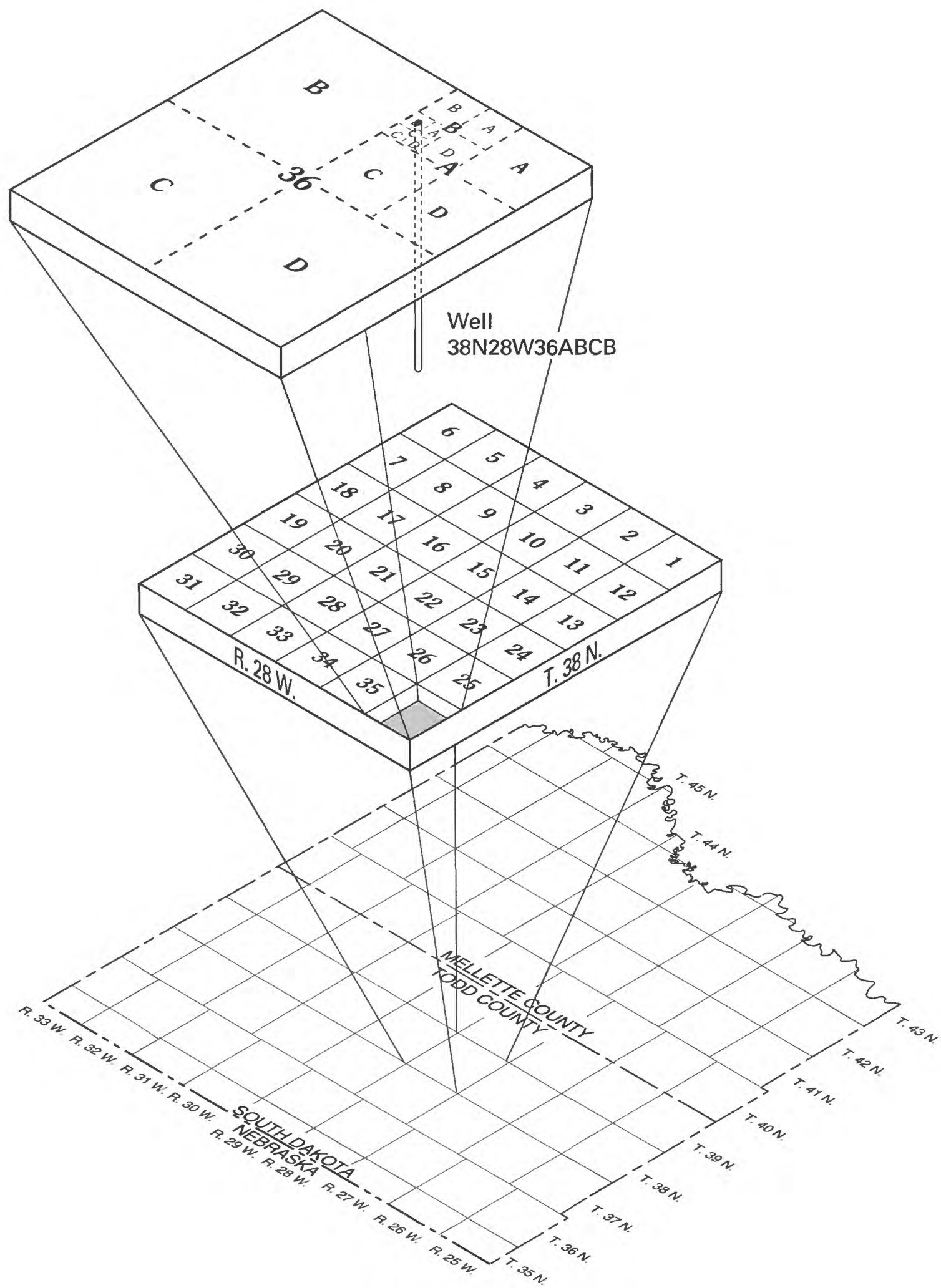

Figure 2. Well-location diagram. The well number consists of the township number, followed by " $\mathrm{N}$," the range number followed by "W," and the section number followed by a maximum of four uppercase letters that indicate, respectively, the $160-, 40-, 10-$, and $21 / 2$-acre tract in which the well is located. These letters are assigned in a counter clockwise direction beginning with "A" in the northeast quarter. A serial number following the last letter is used to distinguish between wells in the same $21 / 2$-acre tract. 
The third identification method is the "other identifier." For the observation wells that were installed by SDGS, the "other identifier" denotes the rig number followed by the year and sequence in which it was drilled. Thus, well R2-94-07 was the seventh well drilled in 1994 by rig \#2. For the private wells that were sampled for the nitrate study, the owner's name is used as the "other identifier."

\section{QUALITY-ASSURANCE PROGRAM}

Quality-assurance samples were collected to evaluate the collection and field-processing methods of water-quality samples and to evaluate the precision and accuracy of the reported analytical results. These quality-assurance samples included blanks and duplicate water samples.

Blank samples, including laboratory and field blanks, were collected and analyzed to identify the presence and magnitude of contamination that potentially could bias analytical results. Laboratory blanks are samples of ultrapure deionized water that are processed through the sampling equipment used in this study within a laboratory or other controlled environment; laboratory blanks are used to identify sample contamination introduced from the sampling equipment. Field blanks are samples of ultrapure deionized water that are processed through the sampling equipment at the field collection site; field blanks are used to identify sample contamination introduced from the sampling equipment, and contamination introduced during collection and processing of samples in the field.

Duplicate samples are two samples collected as close in time as possible and are considered to be essentially identical in composition. After the collection and processing of the duplicate samples in the field, the samples were submitted concurrently to the USGS National Water Quality Laboratory (NWQL) in Arvada, Colorado, for analysis to provide information on the precision of the analytical results.

The precision of the analytical results for a specific constituent can be determined by calculating a standard deviation from the differences of pairs of duplicate measurements. The standard deviation is estimated by the following equation (Taylor, 1987):

$$
S=\sqrt{\frac{\Sigma d^{2}}{2 k}}
$$

where:

$$
\begin{gathered}
S=\text { standard deviation of the difference in con- } \\
\text { centration between duplicate analyses; } \\
d=\text { difference in concentration between each } \\
\text { pair of duplicate analyses; and } \\
k=\text { number of pairs of duplicate analyses. }
\end{gathered}
$$

Precision also can be expressed as a relative standard deviation (RSD), in percent, for a specific constituent. The RSD is calculated from the standard deviation and the mean concentration for all the duplicate analyses as given in the following equation (Taylor, 1987):

$$
R S D=\frac{S}{\bar{x}} \times(100)
$$

where:

$R S D=$ relative standard deviation, in percent;

$S=$ standard deviation; and

$\bar{x}=$ mean of all duplicate concentrations.

The quality-assurance data from the blanks and duplicate samples will be presented in the following sections. Precision data also is provided for the duplicate samples. The statistics for the precision of field duplicates were rounded to two significant figures. For constituents that had concentrations less than the minimum reporting level (censored values), a value of onehalf the reporting level was substituted into the calculations. The data-quality objective for precision of the duplicate field samples is a maximum relative standard deviation of 20 percent.

\section{WATER-QUALITY DATA}

All of the samples collected were analyzed by the USGS NWQL. The collection of ground-water samples in 1990 and 1994 followed methods presented in Wood (1976). Many methods described by Horowitz and others (1994) for the collection and processing of surface-water samples were applied to samples collected after 1994. All ground-water sampling equipment that comes in contact with the sample water was cleaned prior to the sampling trip with a phosphate-free detergent, dilute hydrochloric acid, and deionized water by procedures described by Horowitz and others (1994). After samples were collected from a well, the sampling device and tubing were rinsed with deionized water before sampling the next well. 
In order to assure that a representative sample from the aquifer at each well was collected, the samples were collected after purging a minimum of three casing volumes from the well and after field measurements of specific conductance, $\mathrm{pH}$, temperature, and dissolved oxygen had stabilized (Wood, 1976). Samples were collected from the observation wells using a stainless-steel, submersible pump. Water from the observation wells was passed through a flow-through chamber without coming into contact with the atmosphere so that field measurements were measured in a closed system. The water-quality samples were collected in appropriate bottles directly from a collection tube.

Samples from the domestic wells were collected by connecting a tygon tube to a spigot located so that the samples were not influenced by any pressure, filtration, or chemical treatments. Sample collection was similar to the observation-well collection. The water passed through the instrument chamber without coming into contact with the atmosphere, and the sample water was collected directly from the collection tube.

\section{Reconnaissance Water-Quality Data}

The reconnaissance ground-water sampling program was accomplished during 1990 by the USGS and the Rosebud Sioux Tribe. A total of 100 samples were collected. Most of the sampling was done by personnel from the Office of Water Resources of the Rosebud Sioux Tribe and from the Mellette/Todd Water Resources Coordination Project, with some assistance from USGS personnel. All of the reconnaissance samples were analyzed for field measurements, common ions, and nitrates, and some of the samples were analyzed for trace elements, nutrients, and organic compounds. The locations of the sampling sites are shown in figure 3.

As much information as possible was obtained from driller's logs and the well owner for each of the private wells that was sampled. This information was used to determine the aquifer that supplies each well. Information concerning the well casing, well depth, and formation depths was unavailable or unknown for several of the wells that were sampled. In these cases, the aquifer could usually be determined based on its location and the availability of information from other wells located in the immediate vicinity. However, in some cases, the aquifer could not adequately be deter- mined and no aquifer was assigned to these wells; these sites are listed as unidentified aquifers.

The physical properties and concentrations of inorganic and organic constituents are presented for 18 wells from alluvial aquifers (table 1 in the Supplemental Information section), 29 wells from the Ogallala aquifer (table 2), 14 wells from the Arikaree aquifer (table 3), 10 wells from the White River aquifer (table 4), 19 wells from the Pierre Shale aquifer (table 5), 1 well from the Dakota aquifer (table 6), and 9 wells from unidentified aquifers (table 7).

One quality-assurance field blank was collected for the reconnaissance samples, and the results are shown in table 8. The results of the blank sample indicate that external contamination did not substantially contribute to concentrations of constituents presented in this report.

Five pairs of field duplicate samples were collected and the paired chemical analyses of the field duplicates are presented in table 9 . The estimated precision for each constituent, based on the results of the field duplicates, is presented in table 10 .

The data-quality objective of 20 percent for the precision of field duplicates was exceeded for four of the constituents in table 10 . The relative standard deviations for turbidity and phosphorus exceeded the 20-percent precision and resulted from generally low turbidities and from low phosphorus concentrations. The standard deviation was 0.13 NTU (nephelometric turbidity units) for turbidity and $0.01 \mathrm{mg} / \mathrm{L}$ (milligrams per liter) for phosphorus. The relative standard deviation for ammonia and iron also exceeded the precision of 20 percent. This exceedance resulted from substituting one-half of the minimum reporting level for several censored values, where the concentration for the paired duplicate sample was at the minimum reporting level.

\section{Nitrate Study Water-Quality Data}

High nitrate concentrations, exceeding the U.S. Environmental Protection Agency drinking water maximum contaminant level (MCL) of $10 \mathrm{mg} / \mathrm{L}$ (U.S. Environmental Protection Agency, 1994), were detected in an area in south central Todd County (fig. 4) during the reconnaissance sampling. The high nitrate concentrations had also been noted by the Rosebud Sioux Tribe (Huq, 1989) and by the SDGS (Hammond, 1994). 


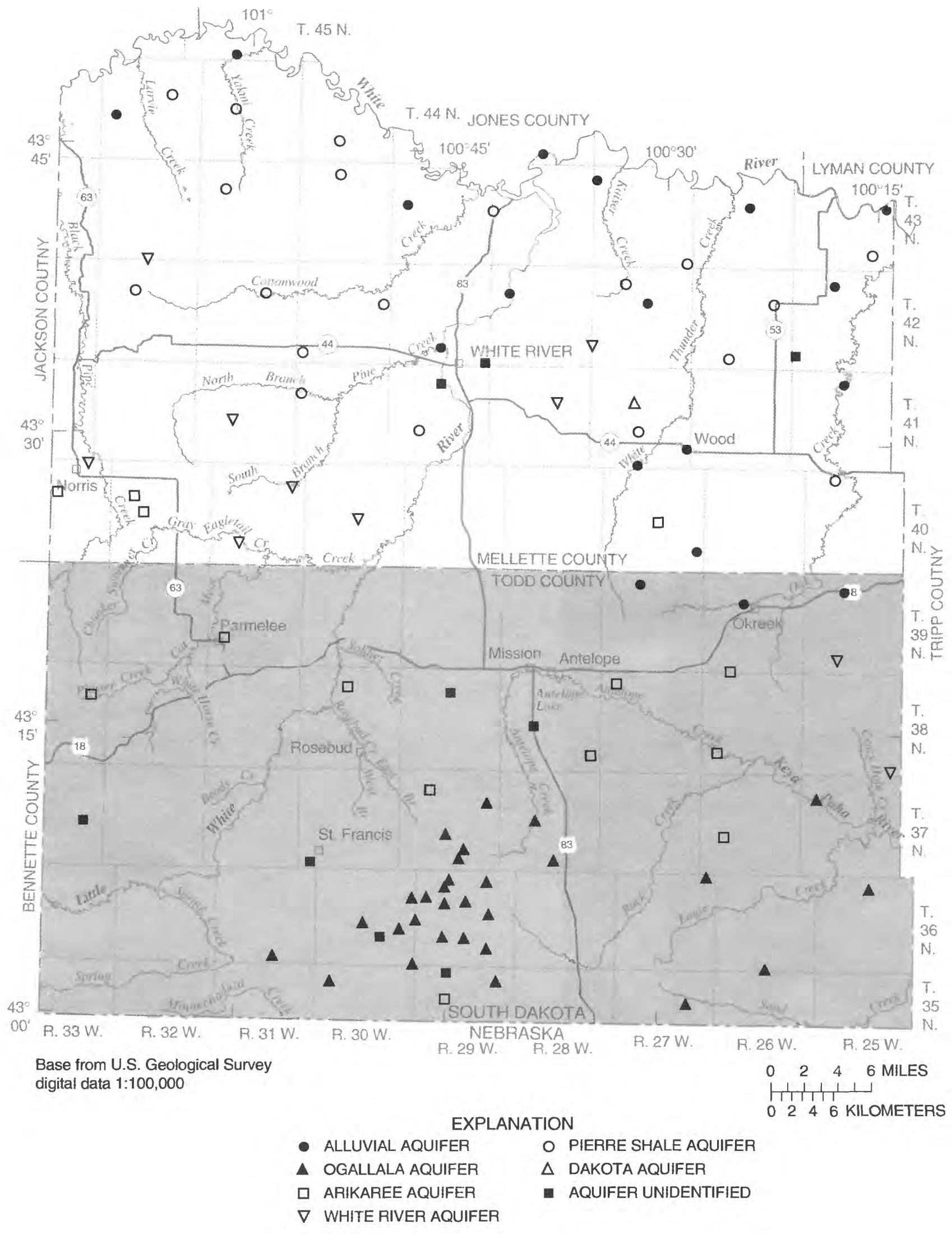

Figure 3. Location of sites sampled during the reconnaissance water-quality program. 


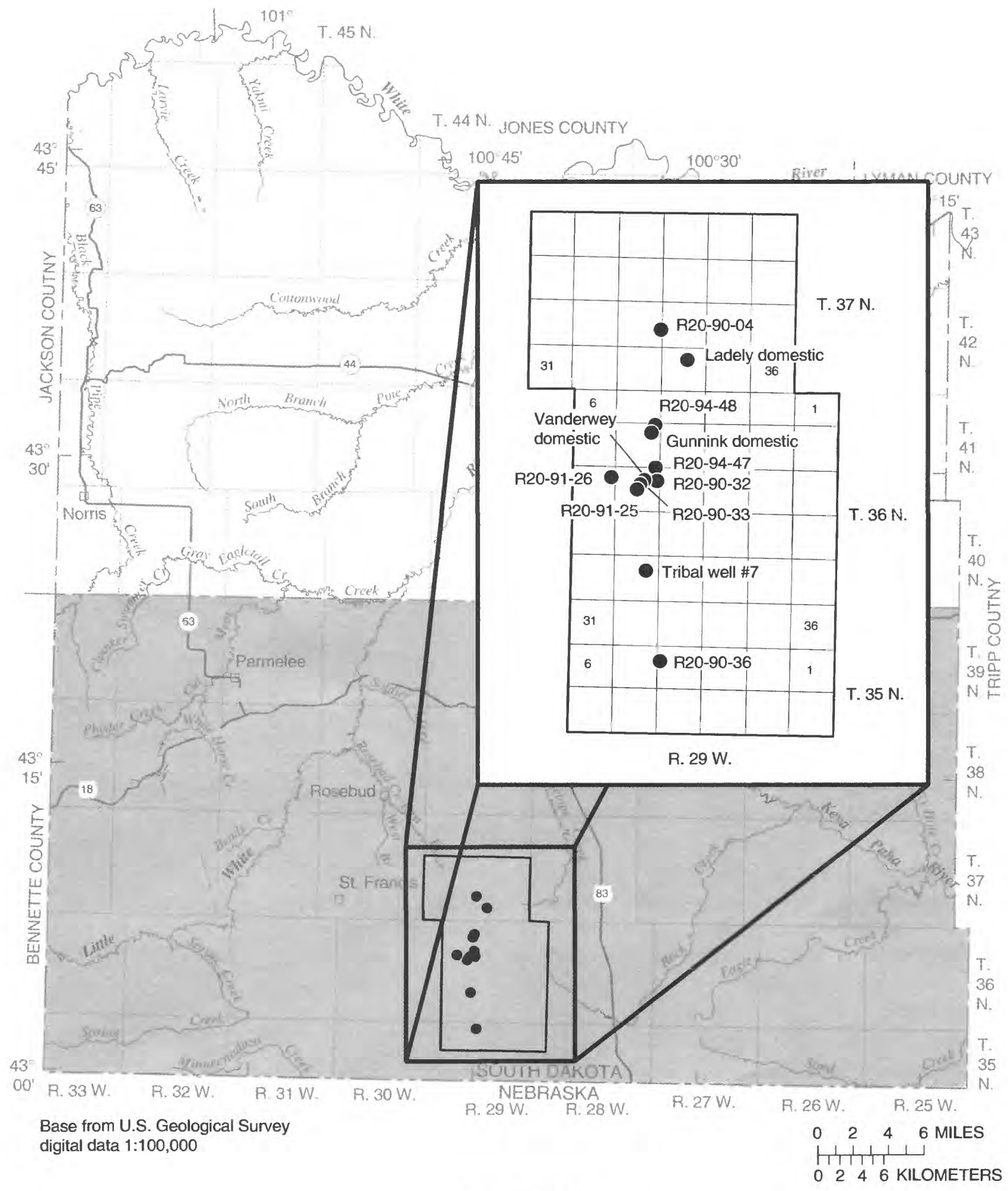

EXPLANATION

- GROUND-WATER SAMPLING SITE

Figure 4. Location of sites sampled for the nitrate study. 
The USGS investigated nitrate concentrations in part of Todd County in independent studies during 1990-91 and during 1994 in cooperation with the Rosebud Sioux Tribe and the SDGS. The purpose of these investigations was to monitor areas that had nitrate concentrations greater than $5 \mathrm{mg} / \mathrm{L}$ (as determined during previous studies) and to determine the source of nitrate contamination. The water-quality data collected for these nitrate investigations are presented in this section. The interpretive results of the studies have been informally presented to the Rosebud Sioux Tribe and will not be presented here.

A total of 19 ground-water samples were collected at 12 sites (fig. 4). The sites included eight observation wells installed by the SDGS, one Tribal monitoring well, and three private domestic wells. All of the wells sampled for the nitrate study were completed in the Ogallala aquifer. The wells were sampled by field personnel from the USGS, with some assistance from field personnel from the Office of Water Resources for the Rosebud Sioux Tribe.

Four ground-water samples were collected during 1990, six during 1991, and nine during 1994. The 1990 samples were analyzed for field measurements, common ions, and nitrates. One site was analyzed for trace elements and organic compounds. These same sites were re-sampled in 1991, along with two additional sites. The 1991 samples were analyzed for field measurements, nitrate concentrations, and nitrogenisotope ratios. Two additional observation wells were installed specifically for the nitrate project in 1994. The 1994 samples were analyzed for field measurements and concentrations of chloride, nitrate, and Methylene Blue Active Substance (MBAS). The analytical results of all the samples collected from 1990-94 by the USGS are presented in table 11.

One field blank and one field duplicate were collected during 1994 for quality-assurance purposes. The chemical analyses of the blank sample are presented in table 12, and the chemical analyses of the paired duplicate samples are presented in table 13. The results of the blank sample indicate that external contamination did not substantially contribute to concentrations of constituents presented in this report. The results of the duplicate sample pair indicate reasonable consistency between the analyses. Precision statistics were not calculated for the duplicate samples because only one pair was collected.

\section{Observation Well Water-Quality Data}

Fifty-six observation wells were drilled and installed between 1992 and 1996 for the Water Resources Investigation of Mellette and Todd Counties. All completed observation wells have 2-inch PVC casing and screen. The wells were gravel packed with washed Platte River sand from Grand Island, Nebraska, and were sealed with bentonite to about 20 feet below land surface and with neat cement from the top of the bentonite to the land surface. The wells were developed using compressed air, after which 5-feet-tall metal protectors were installed over the top of each well.

Water samples were collected from as many of these observation wells as possible during 1994 and 1996. Wells were not sampled if their casing was bent or if the wells did not develop properly. All samples for the observation-well sampling program were collected by USGS personnel. These samples were analyzed for field measurements, common ions, nutrients, trace elements, and radiochemicals. Additionally, bacteria samples (fecal coliform and fecal streptococci) were collected at wells with depths less than 100 feet below land surface; triazine herbicide samples were collected at wells with depths less than 150 feet below land surface. Thirty samples were collected during 1994, and 17 samples were collected during 1996. The locations of the sampling sites are shown in figure 5 .

The physical properties and concentrations of inorganic and organic constituents are presented for 5 wells from alluvial aquifers (table 14), 15 wells from the Ogallala aquifer (table 15), 18 wells from the Arikaree aquifer (table 16), and 9 wells from the White River aquifer (table 17).

Two quality-assurance laboratory blanks and two quality-assurance field blanks were collected during the observation-well sampling program. The results of the blank samples are shown in table 18. Low-level analyses were performed on the laboratory blanks. The results of all analyzed constituents in the laboratory blanks were below the reporting limits of the field samples. The results of the blank samples indicate that external contamination did not substantially contribute to concentrations of constituents presented in this report. Three pairs of duplicate samples were collected in the field for this sampling program. The paired chemical analyses of the field duplicates are presented in table 19 and the estimated precision for each constituent based on the results of the field duplicates is presented in table 20 . 


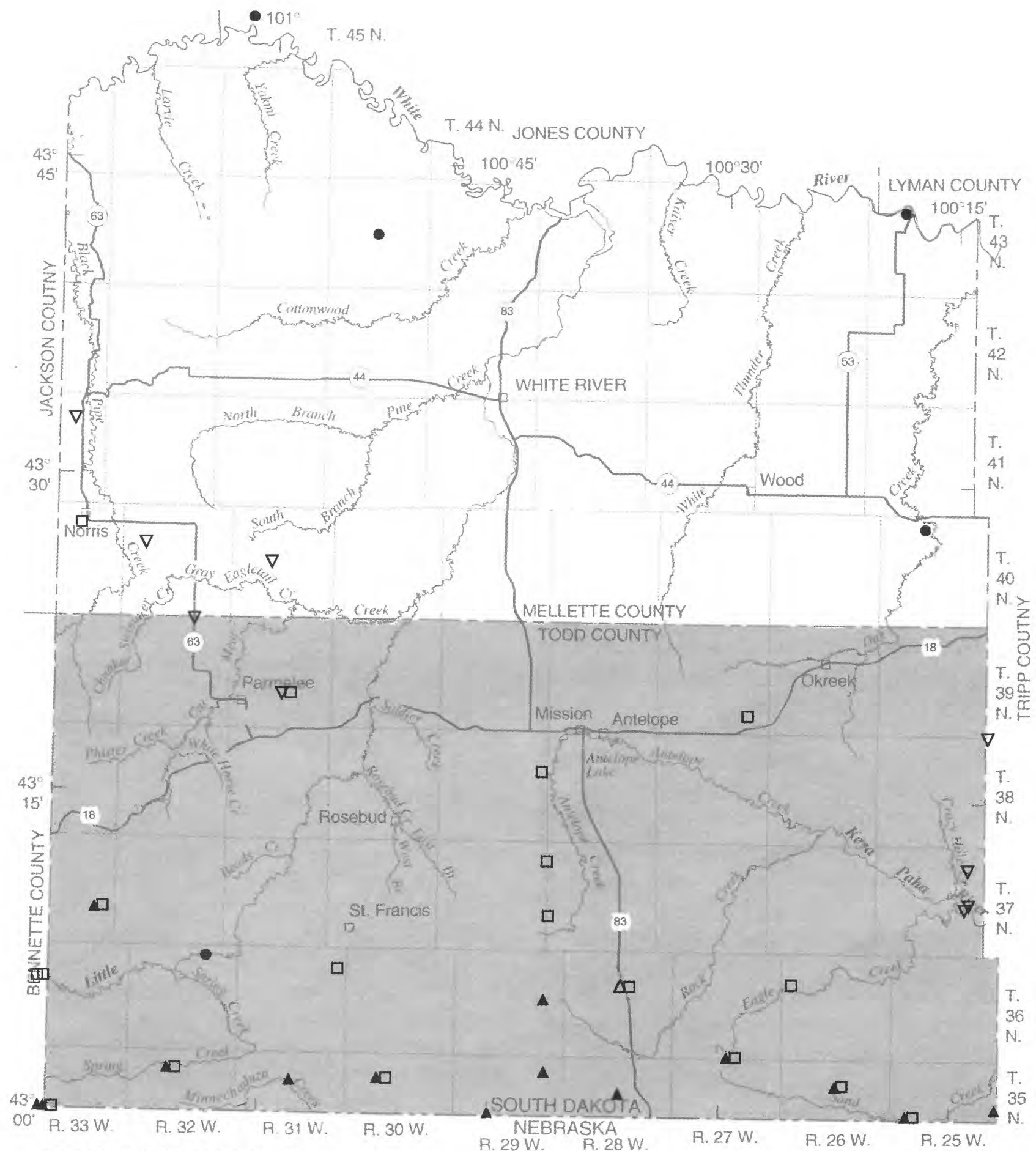

Base from U.S. Geological Survey digital data 1:100,000

$\begin{array}{llll}0 & 2 & 4 & 6\end{array}$ MILES

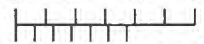

0246 KILOMETERS

EXPLANATION

- Alluvial aQUifer

- Ogallala AQUifer

口 ARIKAREE AQUIFER

$\nabla$ WHITE RIVER AQUIFER

Figure 5. Location of sites sampled during the observation well water-quality program. 
The data-quality objective of 20 percent for the precision of field duplicates was exceeded for two of the constituents in table 20 . The relative standard deviation for selenium exceeded the 20-percent precision because of low concentrations. The large discrepancy of iron concentrations between one duplicate sample set accounts for the high relative standard deviation. The reason(s) for this discrepancy in iron concentrations is unknown but it possibly could have resulted from a data entry error. Because none of the other constituents for this duplicate sample set were in disagreement, the discrepancy between iron concentrations must be an isolated error.

In tables 14-19, 2 sigma refers to the 2 sigma precision estimate (2SPE), which is a measure of the error of the reported concentration. Generally, there is about 95 percent certainty that the true concentration for a sample is within the range of the reported value plus or minus the $2 \mathrm{SPE}$, with the lower part of that range bounded by zero.

\section{REFERENCES CITED}

Ellis, M.J., Ficken, J.H., and Adolphson, D.G., 1971, Hydrology of the Rosebud Indian Reservation, South
Dakota: U.S. Geological Survey Hydrologic Investigations Atlas HA-335.

Hammond, P.D., 1994, Ground water quality investigation in selected areas of Todd and Mellette Counties, South Dakota: South Dakota Geological Survey Open-File Report 68-UR, $36 \mathrm{p}$.

Horowitz, A.J., Demas, C.R., Fitzgerald, K.K., Miller, T.L., Rickert, D.A., 1994, U.S. Geological Survey protocol for the collection and processing of surface-water samples for the subsequent determination of inorganic constituents in filtered water: U.S. Geological Survey Open-File Report 94-539, 57 p.

Huq, S.Y., 1989, Annual report of the Office of Water Resources of the Rosebud Sioux Tribe, Rosebud, South Dakota.

Taylor, J.K., 1987, Quality assurance of chemical measurements: Chelsea, Mich., Lewis Publishers, 328 p.

U.S. Environmental Protection Agency, 1994, Summary of EPA finalized National primary drinking water regulations: U.S. Environmental Protection Agency Region VIII, $7 \mathrm{p}$.

Wood, W.W., 1976, Guidelines for collection and field analysis of ground-water samples for selected unstable constituents: U.S. Geological Survey Techniques of WaterResources Investigations, book 1, chap. D2, 24 p. 
12 
SUPPLEMENTAL INFORMATION

- PAGE 15 followS- 
14 
Table 1. Physical properties and concentrations of inorganic constituents from reconnaissance sampling of alluvial aquifers [Agency collecting or analyzing sample: RST, Rosebud Sioux Tribe. $\mu \mathrm{S} / \mathrm{cm}$, microsiemens per centimeter; mg/L, milligrams per liter; $\mu \mathrm{g} / \mathrm{L}$, micrograms per liter; deg C, degrees Celsius; NTU, nephelometric turbidity units; <, less than; --, no data]

\begin{tabular}{|c|c|c|c|c|c|c|c|}
\hline $\begin{array}{c}\text { Station } \\
\text { identification } \\
\text { number }\end{array}$ & Local number & County & Date & $\begin{array}{c}\text { Depth of } \\
\text { well } \\
\text { (feet) } \\
(72008)\end{array}$ & $\begin{array}{l}\text { Agency } \\
\text { collecting } \\
\text { sample }\end{array}$ & $\begin{array}{l}\text { Specific } \\
\text { conduct- } \\
\text { ance, } \\
\text { field } \\
(\mu \text { S } / \mathrm{cm}) \\
(00095)\end{array}$ & $\begin{array}{c}\mathrm{pH}, \\
\text { field } \\
\text { (standard } \\
\text { units) } \\
(\mathbf{0 0 4 0 0 )}\end{array}$ \\
\hline 432428100273001 & 40N27W25DCAB & Mellette & $07-31-90$ & 30.0 & RST & 960 & 6.8 \\
\hline 433255100173801 & $41 \mathrm{~N} 25 \mathrm{~W} 3 \mathrm{CCC}$ & Mellette & $10-03-90$ & 30.0 & RST & 4,140 & 7.0 \\
\hline 432728100283001 & 41N27W25DAD & Mellette & $10-03-90$ & 55.0 & RST & $1_{3,450}$ & ${ }^{1} 7.3$ \\
\hline 432858100320002 & $41 \mathrm{~N} 27 \mathrm{~W} 33 \mathrm{DDB}$ & Mellette & $07-31-90$ & 80.0 & RST & 1,800 & 6.9 \\
\hline 433822100175601 & $42 \mathrm{~N} 25 \mathrm{~W} 4 \mathrm{DCC}$ & Mellette & $08-16-90$ & 60.0 & RST & 791 & 7.1 \\
\hline 433716100312101 & $42 \mathrm{~N} 27 \mathrm{~W} 15 \mathrm{BAC}$ & Mellette & $08-14-90$ & 45.0 & RST & 3,460 & 6.6 \\
\hline 433738100411901 & $42 \mathrm{~N} 28 \mathrm{~W} 8 \mathrm{CBC}$ & Mellette & $10-22-90$ & 50.0 & RST & 2,780 & 7.3 \\
\hline 433447100460401 & $42 \mathrm{~N} 29 \mathrm{~W} 27 \mathrm{CDC}$ & Mellette & $08-06-90$ & 48.0 & RST & 1,570 & 7.4 \\
\hline 434216100142101 & $43 \mathrm{~N} 25 \mathrm{~W} 13 \mathrm{ACC}$ & Mellette & $08-16-90$ & 13.0 & RST & 2,010 & 7.4 \\
\hline 434216100240501 & $43 \mathrm{~N} 26 \mathrm{~W} 15 \mathrm{BDD}$ & Mellette & $08-16-90$ & 50.0 & RST & 2,600 & 7.0 \\
\hline 434333100514001 & $43 \mathrm{~N} 27 \mathrm{~W} 6 \mathrm{CCD}$ & Mellette & $08-15-90$ & 30.0 & RST & 1,140 & 7.1 \\
\hline 434214100484002 & 43N29W18ADD2 & Mellette & $09-17-90$ & 55.0 & RST & 1,560 & 6.8 \\
\hline 434457100385801 & $44 \mathrm{~N} 28 \mathrm{~W} 33 \mathrm{ADDA}$ & Mellette & $08-15-90$ & 30.0 & RST & 1,140 & 7.1 \\
\hline 434547101081601 & $44 \mathrm{~N} 32 \mathrm{~W} 19 \mathrm{ADDA}$ & Mellette & $09-05-90$ & 10.0 & RST & 497 & 7.1 \\
\hline 434939101011701 & $45 \mathrm{~N} 31 \mathrm{~W} 34 \mathrm{CBCC}$ & Mellette & $09-05-90$ & 20.0 & RST & 2,390 & 7.0 \\
\hline 432228100170201 & 39N25W 9BAAA6 & Todd & $08-28-90$ & 60.0 & RST & 1,620 & 7.0 \\
\hline 432144100241201 & 39N26W 9CDDA & Todd & $08-28-90$ & 20.0 & RST & 833 & 6.9 \\
\hline 432243100313701 & $39 \mathrm{~N} 27 \mathrm{~W} 4 \mathrm{CCAA} 2$ & Todd & $08-28-90$ & 15.0 & RST & 685 & 7.0 \\
\hline
\end{tabular}


Table 1. Physical properties and concentrations of inorganic constituents from reconnaissance sampling of alluvial aquifers-Continued

\begin{tabular}{|c|c|c|c|c|c|c|c|}
\hline Local number & $\begin{array}{l}\text { Temper- } \\
\text { ature, } \\
\text { water } \\
\text { (deg C) } \\
(00010)\end{array}$ & $\begin{array}{c}\text { Turbidity } \\
\text { (NTU) } \\
\text { (00076) }\end{array}$ & $\begin{array}{c}\text { Alkalinity, } \\
\text { lab } \\
\left(\mathrm{mgg}^{2} \mathrm{~L} \text { as }\right. \\
\left.\mathrm{CaCO}_{3}\right) \\
(90410)\end{array}$ & $\begin{array}{c}\text { Calcium, } \\
\text { dissolved } \\
\text { (mg/L } \\
\text { as Ca) } \\
(00915)\end{array}$ & $\begin{array}{c}\text { Magnesium, } \\
\text { dissolved } \\
\text { (mg/L } \\
\text { as Mg) } \\
(00925)\end{array}$ & $\begin{array}{c}\text { Sodium, } \\
\text { dissolved } \\
\text { (mg/L } \\
\text { as Na) } \\
(00930)\end{array}$ & $\begin{array}{l}\text { Sodium, } \\
\text { percent } \\
(00932)\end{array}$ \\
\hline 40N27W25DCAB & 15.0 & 0.20 & 461 & 140 & 7.8 & 72 & 28 \\
\hline $41 \mathrm{~N} 25 \mathrm{~W} 3 \mathrm{CCC}$ & 12.0 & 7.0 & 323 & 420 & 170 & 410 & 33 \\
\hline 41N27W25DAD & -- & 2.4 & 300 & 420 & 55 & 370 & 38 \\
\hline 41N27W33DDB & 14.0 & 0.40 & 327 & 240 & 23 & 130 & 28 \\
\hline 42N25W 4DCC & 16.5 & 0.40 & 263 & 97 & 20 & 38 & 19 \\
\hline $42 \mathrm{~N} 27 \mathrm{~W} 15 \mathrm{BAC}$ & 21.0 & 0.70 & 430 & 520 & 150 & 220 & 20 \\
\hline $42 \mathrm{~N} 28 \mathrm{~W} 8 \mathrm{CBC}$ & -- & 0.80 & 668 & 31 & 4.6 & 570 & 92 \\
\hline $42 \mathrm{~N} 29 \mathrm{~W} 27 \mathrm{CDC}$ & 16.5 & 1.0 & 370 & 34 & 6.2 & 320 & 85 \\
\hline 43N25W13ACC & 18.0 & 0.40 & 408 & 71 & 14 & 370 & 77 \\
\hline 43N26W15BDD & 18.5 & 0.20 & 234 & 190 & 57 & 310 & 48 \\
\hline $43 \mathrm{~N} 27 \mathrm{~W} 6 \mathrm{CCD}$ & 16.5 & 0.60 & 215 & 120 & 14 & 100 & 37 \\
\hline 43N29W18ADD2 & 13.5 & 1.0 & 321 & 200 & 46 & 70 & 18 \\
\hline 44N28W33ADDA & 17.0 & 0.40 & 325 & 63 & 5.7 & 190 & 68 \\
\hline 44N32W19ADDA & 20.5 & 0.60 & 220 & 58 & 6.0 & 38 & 32 \\
\hline $45 \mathrm{~N} 31 \mathrm{~W} 34 \mathrm{CBCC}$ & 17.5 & 0.20 & 256 & 220 & 51 & 350 & 49 \\
\hline 39N25W 9BAAA6 & 15.5 & 0.30 & 316 & 190 & 42 & 110 & 26 \\
\hline 39N26W 9CDDA & 16.0 & 3.5 & 396 & 100 & 7.9 & 67 & 32 \\
\hline 39N27W 4CCAA2 & 16.0 & 0.20 & 360 & 90 & 9.4 & 48 & 27 \\
\hline
\end{tabular}


Table 1. Physical properties and concentrations of inorganic constituents from reconnaissance sampling of alluvial aquifers-Continued

\begin{tabular}{|c|c|c|c|c|c|c|c|}
\hline Local number & $\begin{array}{l}\text { Sodium } \\
\text { adsorption } \\
\text { ratio } \\
(00931)\end{array}$ & $\begin{array}{l}\text { Potassium, } \\
\text { dissolved } \\
\text { (mg/L } \\
\text { as K) } \\
(00935)\end{array}$ & $\begin{array}{c}\text { Sulfate, } \\
\text { dissolved } \\
\left(\mathrm{mghl}^{2} \text { as }\right. \\
\left.\mathrm{SO}_{4}\right) \\
(00945)\end{array}$ & $\begin{array}{c}\text { Chloride, } \\
\text { dissolved } \\
\text { (mg/L } \\
\text { as Cl) } \\
(00940)\end{array}$ & $\begin{array}{c}\text { Fluoride, } \\
\text { dissolved } \\
\text { (mg/L } \\
\text { as F) } \\
(00950)\end{array}$ & $\begin{array}{c}\text { Silica, } \\
\text { dissolved } \\
\text { (mghl } \\
\left.\text { as } \mathrm{SiO}_{2}\right) \\
(00955)\end{array}$ & $\begin{array}{c}\text { Solids, } \\
\text { residue } \\
\text { at } 180 \text { deg } \mathrm{C}, \\
\text { dissolved } \\
(\mathrm{mg} / \mathrm{L}) \\
(70300)\end{array}$ \\
\hline 40N27W25DCAB & 2 & 14 & 44 & 21 & 0.30 & 49 & 610 \\
\hline $41 \mathrm{~N} 25 \mathrm{~W} 3 \mathrm{CCC}$ & 4 & 19 & 2,000 & 7.3 & 0.10 & 22 & 3,820 \\
\hline $41 \mathrm{~N} 27 \mathrm{~W} 25 \mathrm{DAD}$ & 5 & 31 & 1,600 & 64 & 0.10 & 42 & 2,960 \\
\hline $41 \mathrm{~N} 27 \mathrm{~W} 33 \mathrm{DDB}$ & 2 & 14 & 650 & 25 & $<0.10$ & 40 & 1,380 \\
\hline $42 \mathrm{~N} 25 \mathrm{~W} 4 \mathrm{DCC}$ & 0.9 & 20 & 150 & 14 & 0.30 & 39 & 532 \\
\hline $42 \mathrm{~N} 27 \mathrm{~W} 15 \mathrm{BAC}$ & 2 & 20 & 1,800 & 24 & 0.10 & 14 & 3,180 \\
\hline $42 \mathrm{~N} 28 \mathrm{~W} 8 \mathrm{CBC}$ & 25 & 11 & 480 & 160 & 2.2 & 27 & 1,720 \\
\hline $42 \mathrm{~N} 29 \mathrm{~W} 27 \mathrm{CDC}$ & 13 & 6.7 & 430 & 12 & 0.20 & 13 & 1,040 \\
\hline $43 \mathrm{~N} 25 \mathrm{~W} 13 \mathrm{ACC}$ & 11 & 8.7 & 600 & 16 & 0.70 & 25 & 1,390 \\
\hline $43 \mathrm{~N} 26 \mathrm{~W} 15 \mathrm{BDD}$ & 5 & 11 & 1,100 & 28 & 0.40 & 15 & 2,020 \\
\hline $43 \mathrm{~N} 27 \mathrm{~W} 6 \mathrm{CCD}$ & 2 & 14 & 280 & 59 & $<0.10$ & 32 & 766 \\
\hline 43N29W18ADD2 & 1 & 19 & 500 & 30 & 0.20 & 35 & 1,180 \\
\hline 44N28W33ADDA & 6 & 8.7 & 250 & 12 & 0.50 & 27 & 764 \\
\hline 44N32W19ADDA & 1 & 8.0 & 32 & 11 & 0.30 & 44 & 320 \\
\hline $45 \mathrm{~N} 31 \mathrm{~W} 34 \mathrm{CBCC}$ & 6 & 19 & 1,100 & 49 & 0.60 & 30 & 1,900 \\
\hline 39N25W 9BAAA6 & 2 & 19 & 530 & 7.1 & 0.80 & 35 & 1,230 \\
\hline 39N26W 9CDDA & 2 & 18 & 41 & 17 & 1.0 & 44 & 523 \\
\hline $39 \mathrm{~N} 27 \mathrm{~W} 4 \mathrm{CCAA} 2$ & 1 & 13 & 9.6 & 8.3 & 0.80 & 44 & 419 \\
\hline
\end{tabular}


Table 1. Physical properties and concentrations of inorganic constituents from reconnaissance sampling of alluvial aquifers-Continued

\begin{tabular}{|c|c|c|c|c|c|c|c|}
\hline Local number & $\begin{array}{c}\text { Nitrogen, } \\
\text { ammonia, } \\
\text { dissolved } \\
\text { (mg/L as N) } \\
\text { (00608) }\end{array}$ & $\begin{array}{c}\text { Nitrogen, } \\
\text { nitrite, } \\
\text { dissolved } \\
\text { (mg/L as N) } \\
(00613)\end{array}$ & $\begin{array}{c}\text { Nitrogen, } \\
\mathrm{NO}_{2}+\mathrm{NO}_{3} \\
\text { dissolved } \\
\text { (mg/L as N) } \\
\text { (00631) }\end{array}$ & $\begin{array}{l}\text { Phosphorus, } \\
\text { ortho, } \\
\text { dissolved } \\
\text { (mg/L as P) } \\
\text { (00671) }\end{array}$ & $\begin{array}{c}\text { Arsenic, } \\
\text { dissolved } \\
(\mu g / L \\
\text { as As) } \\
(01000)\end{array}$ & $\begin{array}{c}\text { Barium, } \\
\text { dissolved } \\
(\mu g / L \\
\text { as Ba) } \\
(01005)\end{array}$ & $\begin{array}{c}\text { Cadmium, } \\
\text { dissolved } \\
(\mu g / L \\
\text { as Cd) } \\
(01025)\end{array}$ \\
\hline 40N27W25DCAB & -- & -- & 0.80 & -- & 8 & 330 & $<1$ \\
\hline $41 \mathrm{~N} 25 \mathrm{~W} 3 \mathrm{CCC}$ & 0.01 & $<0.01$ & 0.50 & $<0.01$ & -- & -- & -- \\
\hline 41N27W25DAD & $<0.01$ & $<0.01$ & 9.20 & 0.04 & -- & -- & -- \\
\hline 41N27W33DDB & -- & -- & 4.20 & -- & -- & -- & -- \\
\hline 42N25W 4DCC & -- & -- & 0.30 & -- & -- & -- & -- \\
\hline $42 \mathrm{~N} 27 \mathrm{~W} 15 \mathrm{BAC}$ & -- & -- & 0.30 & -- & -- & -- & -- \\
\hline $42 \mathrm{~N} 28 \mathrm{~W} 8 \mathrm{CBC}$ & 1.50 & $<0.01$ & 0.10 & 0.01 & -- & -- & -- \\
\hline $42 \mathrm{~N} 29 \mathrm{~W} 27 \mathrm{CDC}$ & -- & -- & $<0.10$ & -- & -- & -- & -- \\
\hline 43N25W13ACC & -- & -- & $<0.10$ & -- & -- & -- & -- \\
\hline 43N26W15BDD & -- & -- & 1.10 & -- & $<1$ & $<100$ & $<1$ \\
\hline $43 \mathrm{~N} 27 \mathrm{~W} 6 \mathrm{CCD}$ & -- & -- & 2.00 & - & -- & -- & -- \\
\hline 43N29W18ADD2 & -- & -- & $<0.10$ & -- & -- & -- & -- \\
\hline 44N28W33ADDA & -- & -- & $<0.10$ & -- & - & -- & -- \\
\hline 44N32W19ADDA & -- & -- & 2.10 & -- & 5 & 180 & $<1$ \\
\hline $45 \mathrm{~N} 31 \mathrm{~W} 34 \mathrm{CBCC}$ & -- & -- & 0.90 & -- & -- & -- & -- \\
\hline 39N25W 9BAAA6 & -- & -- & 3.20 & -- & 2 & 12 & $<1$ \\
\hline 39N26W 9CDDA & -- & -- & $<0.10$ & -- & -- & -- & -- \\
\hline 39N27W 4CCAA2 & -- & -- & 0.70 & -- & -- & -- & -- \\
\hline
\end{tabular}


Table 1. Physical properties and concentrations of inorganic constituents from reconnaissance sampling of alluvial aquifers-Continued

\begin{tabular}{|c|c|c|c|c|c|c|c|}
\hline Local number & $\begin{array}{c}\text { Chromium, } \\
\text { dissolved } \\
(\mu g / L \\
\text { as Cr) } \\
(01030)\end{array}$ & $\begin{array}{c}\text { Iron, } \\
\text { dissolved } \\
(\mu \mathrm{g} / \mathrm{L} \\
\text { as Fe) } \\
(01046)\end{array}$ & $\begin{array}{c}\text { Lead, } \\
\text { dissolved } \\
(\mu \mathrm{g} / \mathrm{L} \\
\mathrm{as} \mathrm{Pb}) \\
(01049)\end{array}$ & $\begin{array}{c}\text { Manganese, } \\
\text { dissolved } \\
(\mu g / L \\
\text { as Mn) } \\
(01056)\end{array}$ & $\begin{array}{c}\text { Mercury, } \\
\text { dissolved } \\
(\mu g / 2 \\
\text { as } \mathrm{Hg}) \\
(71890)\end{array}$ & $\begin{array}{c}\text { Selenium, } \\
\text { dissolved } \\
(\mu g / L \\
\text { as Se) } \\
(01145)\end{array}$ & $\begin{array}{c}\text { Zinc, } \\
\text { dissolved } \\
(\mu g / L \\
\text { as } \mathrm{Zn}) \\
(01090)\end{array}$ \\
\hline 40N27W25DCAB & $<1$ & 4 & 2 & $<1$ & $<0.1$ & 3 & 12 \\
\hline $41 \mathrm{~N} 25 \mathrm{~W} 3 \mathrm{CCC}$ & -- & -- & -- & -- & -- & -. & -. \\
\hline $41 \mathrm{~N} 27 \mathrm{~W} 25 \mathrm{DAD}$ & -- & -- & -- & -. & -- & -- & -- \\
\hline $41 \mathrm{~N} 27 \mathrm{~W} 33 \mathrm{DDB}$ & -- & -- & -- & - & -- & -- & -- \\
\hline $42 \mathrm{~N} 25 \mathrm{~W} 4 \mathrm{DCC}$ & -- & -- & -- & -- & -- & -- & -- \\
\hline $42 \mathrm{~N} 27 \mathrm{~W} 15 \mathrm{BAC}$ & -- & -- & -- & -- & -- & -- & -- \\
\hline $42 \mathrm{~N} 28 \mathrm{~W} 8 \mathrm{CBC}$ & -. & - & -- & - & -. & -. & -- \\
\hline $42 \mathrm{~N} 29 \mathrm{~W} 27 \mathrm{CDC}$ & -- & -- & -- & - & -- & -- & -- \\
\hline $43 \mathrm{~N} 25 \mathrm{~W} 13 \mathrm{ACC}$ &.- & -- & -- & -- & -- & -- & -- \\
\hline 43N26W15BDD & $<1$ & 20 & $<1$ & $<10$ & $<0.1$ & 6 & 30 \\
\hline $43 \mathrm{~N} 27 \mathrm{~W} 6 \mathrm{CCD}$ & -- & -- & -- & -- & -- & -- & -- \\
\hline 43N29W18ADD2 & -- & -- & - & - & -- & -- & -- \\
\hline 44N28W33ADDA & -- & -- & -- & -- & -- & -. & - \\
\hline 44N32W19ADDA & 1 & 6 & $<1$ & 1 & $<0.1$ & 3 & 25 \\
\hline 45N31W34CBCC & -- & -- & -- & -- & -- & -- & -- \\
\hline 39N25W 9BAAA6 & 2 & 12 & $<1$ & 7 & $<0.1$ & 25 & 120 \\
\hline 39N26W 9CDDA & - & -- & -- & -- & -- & -- & -- \\
\hline $39 \mathrm{~N} 27 \mathrm{~W} 4 \mathrm{CCAA} 2$ & -- & -- & -- & - & -- & -- & - \\
\hline
\end{tabular}

${ }^{1}$ Laboratory value is given because field value was missing. 
Table 2. Physical properties and concentrations of inorganic and organic constituents from reconnaissance sampling of the Ogallala aquifer

[Agency collecting or analyzing sample: RST, Rosebud Sioux Tribe; USGS, U.S. Geological Survey. $\mu$ S/cm, microsiemens per centimeter; mg/L, milligrams per liter; $\mu \mathrm{g} / \mathrm{L}$, micrograms per liter; deg C, degrees Celsius; NTU, nephelometric turbidity units; unftrd, unfiltered; recover, recoverable; $<$, less than; --, no data]

\begin{tabular}{|c|c|c|c|c|c|c|c|c|}
\hline Station number & Local number & County & Date & $\begin{array}{c}\text { Depth of } \\
\text { well } \\
\text { (feet) } \\
\text { (72008) }\end{array}$ & $\begin{array}{l}\text { Agency } \\
\text { collecting } \\
\text { sample }\end{array}$ & $\begin{array}{c}\text { Specific } \\
\text { conduct- } \\
\text { ance, } \\
\text { field } \\
(\mu \mathrm{S} / \mathrm{cm}) \\
(00095)\end{array}$ & $\begin{array}{c}\mathrm{pH}, \\
\text { field } \\
\text { (standard } \\
\text { units) } \\
(\mathbf{0 0 4 0 0 )}\end{array}$ & $\begin{array}{c}\text { Temper- } \\
\text { ature, } \\
\text { water } \\
\text { (deg C) } \\
\text { (00010) }\end{array}$ \\
\hline 430057100275401 & $35 \mathrm{~N} 27 \mathrm{~W} 14 \mathrm{BAAB}$ & Todd & $10-23-90$ & 84.2 & RST & 387 & 7.4 & 12.5 \\
\hline 430154100411801 & $35 \mathrm{~N} 29 \mathrm{~W}$ 2DDDD & Todd & $07-18-90$ & 44.4 & USGS & 911 & 6.9 & 12.0 \\
\hline 430159100531001 & $35 \mathrm{~N} 30 \mathrm{~W}$ 6DDDD & Todd & $08-01-90$ & 83.5 & USGS & 334 & 7.4 & 13.0 \\
\hline 430704100145901 & $36 \mathrm{~N} 25 \mathrm{~W} 10 \mathrm{BABB}$ & Todd & $08-29-90$ & 30.0 & USGS & 326 & 7.5 & 16.5 \\
\hline 430245100223101 & $36 \mathrm{~N} 26 \mathrm{~W} 34 \mathrm{CCC}$ & Todd & $10-23-90$ & -. & RST & 371 & 7.5 & 13.0 \\
\hline 430728100135801 & $36 \mathrm{~N} 27 \mathrm{~W} 1 \mathrm{BDDD}$ & Todd & $08-29-90$ & 58.1 & RST & $1_{389}$ & 7.2 & 12.5 \\
\hline 430712100421301 & $36 \mathrm{~N} 29 \mathrm{~W} 2 \mathrm{CDCC}$ & Todd & 09-19-90 & 200.0 & RST & 341 & 7.6 & 12.0 \\
\hline 430714100445001 & $36 \mathrm{~N} 29 \mathrm{~W} 4 \mathrm{CCBC}$ & Todd & $07-19-90$ & 150.0 & USGS & 336 & 7.4 & 14.0 \\
\hline 430620100462301 & $36 \mathrm{~N} 29 \mathrm{~W} 7 \mathrm{DCC}$ & Todd & $10-23-90$ & -- & RST & ${ }^{1} 303$ & 17.7 & -- \\
\hline 430652100450601 & $36 \mathrm{~N} 29 \mathrm{~W} 8 \mathrm{~A}$ & Todd & 09-19-90 & 190.0 & RST & $1_{417}$ & ${ }^{17.6}$ & -- \\
\hline 430530100422501 & $36 \mathrm{~N} 29 \mathrm{~W} 14 \mathrm{CDAB}$ & Todd & $09-19-90$ & 200.0 & RST & 351 & 7.6 & 13.0 \\
\hline 430555100433601 & $36 \mathrm{~N} 29 \mathrm{~W} 15 \mathrm{BBB}$ & Todd & $10-15-90$ & 160.0 & RST & 352 & 7.6 & 12.5 \\
\hline 430600100450901 & $36 \mathrm{~N} 29 \mathrm{~W} 17 \mathrm{ADBB}$ & Todd & $07-19-90$ & -. & USGS & 743 & 7.2 & 13.5 \\
\hline 430504100471201 & $36 \mathrm{~N} 29 \mathrm{~W} 19 \mathrm{BCBA}$ & Todd & $10-15-90$ & -- & RST & 328 & 7.6 & 12.5 \\
\hline 430340100420501 & $36 \mathrm{~N} 29 \mathrm{~W} 26 \mathrm{CDC}$ & Todd & $09-19-90$ & -- & RST & 381 & 7.4 & 12.0 \\
\hline 430405100434201 & $36 \mathrm{~N} 29 \mathrm{~W} 28 \mathrm{ADA}$ & Todd & $09-19-90$ & 160.0 & RST & 383 & 7.5 & 13.5 \\
\hline 430415100451401 & $36 \mathrm{~N} 29 \mathrm{~W} 29 \mathrm{ACAA}$ & Todd & $07-18-90$ & 133.5 & USGS & 385 & 7.4 & 13.5 \\
\hline 430615100472701 & $36 \mathrm{~N} 30 \mathrm{~W} 12 \mathrm{DDCD}$ & Todd & $10-15-90$ & 285.0 & RST & 342 & ${ }^{1} 7.7$ & -. \\
\hline 430501100504901 & 36N30W21DAAA & Todd & 08-01-90 & 137.0 & USGS & 253 & 7.6 & 12.5 \\
\hline 430418100480801 & $36 \mathrm{~N} 30 \mathrm{~W} 24 \mathrm{CCDB}$ & Todd & $10-15-90$ & -- & RST & 583 & 7.5 & 14.0 \\
\hline 430258100471401 & $36 \mathrm{~N} 30 \mathrm{~W} 36 \mathrm{DDDA}$ & Todd & $08-01-90$ & 123.0 & USGS & 356 & 7.4 & 13.5 \\
\hline 430309100570901 & 36N31W34DBBC & Todd & $10-23-90$ & 90.5 & RST & 342 & 7.4 & 12.0 \\
\hline 431137100185301 & 37N25W 7DAAD & Todd & $08-29-90$ & 100.0 & RST & 780 & 7.9 & 13.5 \\
\hline 431021100384701 & $37 \mathrm{~N} 28 \mathrm{~W} 16 \mathrm{CCDD}$ & Todd & $08-31-90$ & 80.0 & RST & 323 & 7.4 & 13.0 \\
\hline 430809100372401 & $37 \mathrm{~N} 28 \mathrm{~W} 34 \mathrm{BDA} 2$ & Todd & $10-23-90$ & 171.1 & RST & 327 & 7.5 & 12.5 \\
\hline 431116100422001 & $37 \mathrm{~N} 29 \mathrm{~W} 12 \mathrm{CCD}$ & Todd & $07-18-90$ & -- & USGS & 365 & 7.3 & 14.5 \\
\hline 430937100445801 & 37N29W21DDAD & Todd & $10-15-90$ & -- & RST & 754 & 7.3 & 13.0 \\
\hline 430852100435001 & $37 \mathrm{~N} 29 \mathrm{~W} 27 \mathrm{DACD}$ & Todd & $10-15-90$ & 180.0 & RST & 255 & 7.8 & 12.5 \\
\hline 430823100441201 & $37 \mathrm{~N} 29 \mathrm{~W} 34 \mathrm{ACA}$ & Todd & $07-19-90$ & 150.0 & USGS & 710 & 6.9 & 14.5 \\
\hline
\end{tabular}


Table 2. Physical properties and concentrations of inorganic and organic constituents from reconnaissance sampling of the Ogallala aquifer-Continued

\begin{tabular}{|c|c|c|c|c|c|c|c|c|c|}
\hline Local number & $\begin{array}{l}\text { Turbidity } \\
\text { (NTU) } \\
\text { (00076) }\end{array}$ & $\begin{array}{c}\text { Alkalinity, } \\
\text { lab } \\
(\mathrm{mg} / \mathrm{L} \text { as } \\
\left.\mathrm{CaCO}_{3}\right) \\
(90410)\end{array}$ & $\begin{array}{c}\text { Calcium, } \\
\text { dissolved } \\
\text { (mg/h } \\
\text { as Ca) } \\
(00915)\end{array}$ & $\begin{array}{c}\text { Magne- } \\
\text { sium, } \\
\text { dissolved } \\
(\mathrm{mg} / \mathrm{L} \\
\text { as } \mathrm{Mg}) \\
(00925)\end{array}$ & $\begin{array}{c}\text { Sodium, } \\
\text { dissolved } \\
\text { (mg/h } \\
\text { as Na) } \\
(00930)\end{array}$ & $\begin{array}{l}\text { Sodium, } \\
\text { percent } \\
\text { (00932) }\end{array}$ & $\begin{array}{c}\text { Sodium } \\
\text { adsorp- } \\
\text { tion } \\
\text { ratio } \\
(00931)\end{array}$ & $\begin{array}{c}\text { Potas- } \\
\text { sium, } \\
\text { dissolved } \\
\text { (mg/L } \\
\text { as K) } \\
(00935)\end{array}$ & $\begin{array}{c}\text { Sulfate, } \\
\text { dissolved } \\
(\mathrm{mg} / \mathrm{L} \\
\text { as SO } \\
(00945)\end{array}$ \\
\hline 35N27W14BAAB & 35 & 199 & 52 & 10 & 9.9 & 10 & 0.3 & 10 & 5.7 \\
\hline 35N29W 2DDDD & 0.70 & 330 & 89 & 8.8 & 97 & 43 & 3 & 16 & 86 \\
\hline 35N30W 6DDDD & 0.40 & 174 & 62 & 2.6 & 3.0 & 4 & 0.1 & 6.5 & $<1.0$ \\
\hline 36N25W10BABB & 0.20 & 153 & 32 & 4.8 & 29 & 36 & 1 & 9.8 & 14 \\
\hline $36 \mathrm{~N} 26 \mathrm{~W} 34 \mathrm{CCC}$ & 0.50 & 179 & 41 & 7.2 & 18 & 21 & 0.7 & 12 & 12 \\
\hline 36N27W 1BDDD & 42 & 184 & 77 & 2.4 & 1.7 & 2 & 0.0 & 5.9 & 14 \\
\hline $36 \mathrm{~N} 29 \mathrm{~W} 2 \mathrm{CDCC}$ & 3.0 & 176 & 48 & 8.1 & 9.4 & 11 & 0.3 & 10 & 6.3 \\
\hline $36 \mathrm{~N} 29 \mathrm{~W} 4 \mathrm{CCBC}$ & 0.30 & 160 & 50 & 7.7 & 5.8 & 7 & 0.2 & 6.9 & 2.8 \\
\hline 36N29W 7DCC & 0.20 & 158 & 44 & 7.7 & 3.9 & 5 & 0.1 & 8.8 & 1.6 \\
\hline $36 \mathrm{~N} 29 \mathrm{~W} 8 \mathrm{~A}$ & 0.40 & 175 & 61 & 10 & 5.3 & 5 & 0.2 & 7.3 & 5.7 \\
\hline 36N29W14CDAB & 3.0 & 163 & 41 & 9.5 & 14 & 16 & 0.5 & 11 & 9.7 \\
\hline $36 \mathrm{~N} 29 \mathrm{~W} 15 \mathrm{BBB}$ & 0.40 & 177 & 51 & 9.5 & 5.3 & 6 & 0.2 & 7.7 & 3.2 \\
\hline 36N29W17ADBB & 0.30 & 191 & 110 & 13 & 8.9 & 5 & 0.2 & 9.9 & 7.8 \\
\hline 36N29W19BCBA & 0.50 & 161 & 48 & 8.9 & 3.6 & 5 & 0.1 & 7.4 & 2.0 \\
\hline $36 \mathrm{~N} 29 \mathrm{~W} 26 \mathrm{CDC}$ & 0.60 & 190 & 57 & 9.8 & 5.1 & 5 & 0.2 & 7.5 & 2.1 \\
\hline $36 \mathrm{~N} 29 \mathrm{~W} 28 \mathrm{ADA}$ & 0.30 & 175 & 59 & 6.6 & 6.5 & 7 & 0.2 & 8.8 & 6.9 \\
\hline 36N29W29ACAA & 0.40 & 157 & 35 & 5.3 & 37 & 40 & 2 & 9.5 & 16 \\
\hline 36N30W 12DDCD & 0.30 & 165 & 53 & 7.5 & 4.5 & 5 & 0.2 & 8.7 & 3.9 \\
\hline 36N30W21DAAA & 0.70 & 131 & 40 & 6.4 & 1.1 & 2 & 0.0 & 6.3 & 1.6 \\
\hline 36N30W24CCDB & 0.20 & 186 & 81 & 14 & 11 & 8 & 0.3 & 10 & 11 \\
\hline 36N30W36DDDA & 0.40 & 173 & 49 & 10 & 7.4 & 9 & 0.3 & 6.9 & 5.5 \\
\hline 36N31W34DBBC & 0.40 & 179 & 54 & 7.0 & 7.8 & 9 & 0.3 & 7.3 & 1.8 \\
\hline 37N25W 7DAAD & 0.40 & 340 & 7.3 & 0.78 & 180 & 93 & 17 & 8.0 & 63 \\
\hline 37N28W16CCDD & 0.30 & 165 & 46 & 9.7 & 5.8 & 7 & 0.2 & 7.6 & 2.2 \\
\hline 37N28W34BDA2 & 3.4 & 168 & 43 & 11 & 4.1 & 5 & 0.1 & 7.9 & 2.1 \\
\hline $37 \mathrm{~N} 29 \mathrm{~W} 12 \mathrm{CCD}$ & 2.4 & 174 & 47 & 9.2 & 13 & 14 & 0.5 & 9.9 & 9.6 \\
\hline 37N29W21DDAD & 0.20 & 221 & 100 & 17 & 9.3 & 6 & 0.2 & 10 & 17 \\
\hline 37N29W27DACD & 0.30 & 129 & 36 & 6.8 & 2.8 & 5 & 0.1 & 6.7 & 2.3 \\
\hline 37N29W34ACA & 0.30 & 153 & 120 & 4.3 & 3.9 & 3 & 0.1 & 8.8 & 28 \\
\hline
\end{tabular}


Table 2. Physical properties and concentrations of inorganic and organic constituents from reconnaissance sampling of the Ogallala aquifer-Continued

\begin{tabular}{|c|c|c|c|c|c|c|c|c|}
\hline Local number & $\begin{array}{l}\text { Chloride, } \\
\text { dissolved } \\
\text { (mg/L. } \\
\text { as Cl) } \\
(00940)\end{array}$ & $\begin{array}{c}\text { Fluoride, } \\
\text { dissolved } \\
\text { (mg/L } \\
\text { as F) } \\
(00950)\end{array}$ & $\begin{array}{l}\text { Silica, } \\
\text { dissolved } \\
\text { (mg/L. } \\
\left.\text { as } \mathrm{SiO}_{2}\right) \\
(00955)\end{array}$ & $\begin{array}{c}\text { Solids, } \\
\text { residue } \\
\text { at } 180 \\
\text { deg C, } \\
\text { dissolved } \\
(\mathrm{mg} / \mathrm{L}) \\
(70300)\end{array}$ & $\begin{array}{c}\text { Nitrogen, } \\
\text { ammonia, } \\
\text { dissolved } \\
\text { (mg/L } \\
\text { as N) } \\
(00608)\end{array}$ & $\begin{array}{c}\text { Nitrogen, } \\
\text { nitrite, } \\
\text { dissolved } \\
\text { (mg/L } \\
\text { as N) } \\
(00613)\end{array}$ & $\begin{array}{c}\text { Nitrogen, } \\
\mathrm{NO}_{2}+\mathrm{NO}_{3} \\
\text { dissolved } \\
\text { (mg/L } \\
\text { as N) } \\
(00631)\end{array}$ & $\begin{array}{c}\text { Phos- } \\
\text { phorus, } \\
\text { ortho, } \\
\text { dissolvec } \\
\text { (mg/L } \\
\text { as P) } \\
\text { (00671) }\end{array}$ \\
\hline 35N27W14BAAB & 3.3 & 0.30 & 67 & 262 & 0.02 & $<0.01$ & 0.70 & 0.01 \\
\hline $35 \mathrm{~N} 29 \mathrm{~W} 2 \mathrm{DDDD}$ & 8.1 & 1.1 & 57 & 619 & -- & -- & 16.0 & -- \\
\hline 35N30W 6DDDD & 0.20 & 0.40 & 52 & 233 & -- & - & 0.70 & -- \\
\hline $36 \mathrm{~N} 25 \mathrm{~W} 10 \mathrm{BABB}$ & 3.8 & 0.70 & 67 & 237 & -- & -- & 1.80 & -- \\
\hline $36 \mathrm{~N} 26 \mathrm{~W} 34 \mathrm{CCC}$ & 3.6 & 0.30 & 66 & 261 & 0.02 & $<0.01$ & 1.10 & 0.02 \\
\hline $36 \mathrm{~N} 27 \mathrm{~W} 1 \mathrm{BDDD}$ & 4.2 & 0.40 & 51 & 279 & -- & -- & 3.00 & -- \\
\hline $36 \mathrm{~N} 29 \mathrm{~W} 2 \mathrm{CDCC}$ & 4.1 & 0.40 & 64 & 239 & -- & -- & 0.90 & -. \\
\hline $36 \mathrm{~N} 29 \mathrm{~W} 4 \mathrm{CCBC}$ & 3.6 & 0.30 & 58 & 243 & -- & - & 2.80 & -- \\
\hline $36 \mathrm{~N} 29 \mathrm{~W} 7 \mathrm{DCC}$ & 2.9 & 0.20 & 57 & 214 & 0.02 & $<0.01$ & 0.90 & 0.02 \\
\hline $36 \mathrm{~N} 29 \mathrm{~W} 8 \mathrm{~A}$ & 8.0 & 0.30 & 54 & 282 & -. & -- & 9.10 & -- \\
\hline $36 \mathrm{~N} 29 \mathrm{~W} 14 \mathrm{CDAB}$ & 2.9 & 0.40 & 67 & 255 & -- & -- & 3.40 & -- \\
\hline $36 \mathrm{~N} 29 \mathrm{~W} 15 \mathrm{BBB}$ & 4.0 & 0.20 & 58 & 241 & $<0.01$ & $<0.01$ & 1.60 & 0.01 \\
\hline 36N29W17ADBB & 21 & 0.30 & 54 & 492 & -- & -- & 29.0 & -- \\
\hline $36 \mathrm{~N} 29 \mathrm{~W} 19 \mathrm{BCBA}$ & 3.3 & 0.30 & 57 & 230 & $<0.01$ & $<0.01$ & 2.20 & 0.02 \\
\hline $36 \mathrm{~N} 29 \mathrm{~W} 26 \mathrm{CDC}$ & 3.2 & 0.30 & 60 & 235 & -- & -- & 1.20 & -- \\
\hline $36 \mathrm{~N} 29 \mathrm{~W} 28 \mathrm{ADA}$ & 5.6 & 0.30 & 59 & 256 & -- & -- & 3.50 & -- \\
\hline $36 \mathrm{~N} 29 \mathrm{~W} 29 \mathrm{ACAA}$ & 2.8 & 0.50 & 69 & 279 & -. & -- & 1.10 &.- \\
\hline $36 \mathrm{~N} 30 \mathrm{~W} 12 \mathrm{DDCD}$ & 5.6 & 0.20 & 56 & 239 & $<0.01$ & $<0.01$ & 1.60 & $<0.01$ \\
\hline $36 \mathrm{~N} 30 \mathrm{~W} 21 \mathrm{DAAA}$ & 0.20 & 0.30 & 53 & 189 & -- & -- & 0.70 & -- \\
\hline $36 \mathrm{~N} 30 \mathrm{~W} 24 \mathrm{CCDB}$ & 20 & 0.20 & 58 & 414 & 0.01 & $<0.01$ & 18.0 & $<0.01$ \\
\hline 36N30W36DDDA & 4.7 & 0.20 & 65 & 258 & -- & - & 1.60 & -- \\
\hline $36 \mathrm{~N} 31 \mathrm{~W} 34 \mathrm{DBBC}$ & 3.0 & 0.30 & 53 & 231 & 0.03 & $<0.01$ & 0.60 & 0.04 \\
\hline 37N25W 7DAAD & 15 & 0.90 & 58 & 505 & -. & -- & $<0.10$ & -- \\
\hline $37 \mathrm{~N} 28 \mathrm{~W} 16 \mathrm{CCDD}$ & 2.7 & 0.30 & -- & -- & -- & -- & 1.10 & -- \\
\hline $37 \mathrm{~N} 28 \mathrm{~W} 34 \mathrm{BDA} 2$ & 3.5 & 0.20 & 61 & 217 & 0.03 & $<0.01$ & 1.00 & 0.02 \\
\hline $37 \mathrm{~N} 29 \mathrm{~W} 12 \mathrm{CCD}$ & 3.1 & 0.30 & 65 & 249 & -- & -- & 1.20 & -- \\
\hline 37N29W21DDAD & 20 & 0.20 & 55 & 530 & $<0.01$ & $<0.01$ & 30.0 & $<0.01$ \\
\hline 37N29W27DACD & 2.5 & 0.20 & 59 & 190 & $<0.01$ & $<0.01$ & 0.90 & $<0.01$ \\
\hline $37 \mathrm{~N} 29 \mathrm{~W} 34 \mathrm{ACA}$ & 28 & 0.30 & 55 & 598 & -- & -- & 44.0 & -- \\
\hline
\end{tabular}


Table 2. Physical properties and concentrations of inorganic and organic constituents from reconnaissance sampling of the Ogallala aquifer-Continued

\begin{tabular}{|c|c|c|c|c|c|c|c|c|}
\hline Local number & $\begin{array}{c}\text { Arsenic, } \\
\text { dissolved } \\
(\mu g / L \\
\text { as As) } \\
(01000)\end{array}$ & $\begin{array}{c}\text { Barium, } \\
\text { dissolved } \\
(\mu \mathrm{g} / \mathrm{L} \\
\text { as Ba) } \\
(01005)\end{array}$ & $\begin{array}{c}\text { Cadmium, } \\
\text { dissolved } \\
(\mu g / L \\
\text { as Cd) } \\
(01025)\end{array}$ & $\begin{array}{c}\text { Chromium, } \\
\text { dissolved } \\
(\mu \mathrm{g} / \mathrm{L} \\
\text { as Cr) } \\
(01030)\end{array}$ & $\begin{array}{c}\text { Iron, } \\
\text { dissolved } \\
(\mu g / L \\
\text { as Fe) } \\
(01046)\end{array}$ & $\begin{array}{c}\text { Lead, } \\
\text { dissolved } \\
(\mu \mathrm{g} / \mathrm{L} \\
\text { as } \mathrm{Pb}) \\
(01049)\end{array}$ & $\begin{array}{c}\text { Manga- } \\
\text { nese, } \\
\text { dissolved } \\
(\mu g / L \\
\text { as } M n) \\
(01056)\end{array}$ & $\begin{array}{c}\text { Mercury, } \\
\text { dissolved } \\
(\mu \mathrm{g} / \mathrm{L} \\
\text { as } \mathrm{Hg}) \\
(71890)\end{array}$ \\
\hline $35 \mathrm{~N} 27 \mathrm{~W} 14 \mathrm{BAAB}$ & -- & -- & -. & -- & -- & -- & -- & -- \\
\hline $35 \mathrm{~N} 29 \mathrm{~W} 2 \mathrm{DDDD}$ & -- & -- & -- & -- & -. & -. & -- & -. \\
\hline 35N30W 6DDDD & -- & -- & -- & -- & -- & -- & -- & .. \\
\hline $36 \mathrm{~N} 25 \mathrm{~W} 10 \mathrm{BABB}$ & -- & -- & -- & -- & -- & -- & -- & -- \\
\hline $36 \mathrm{~N} 26 \mathrm{~W} 34 \mathrm{CCC}$ & -- & -- & -- & -- & -- & -- & -- & -- \\
\hline $36 \mathrm{~N} 27 \mathrm{~W} 1 \mathrm{BDDD}$ & -- & -- & -- & -- & -- & -- & -- & -- \\
\hline $36 \mathrm{~N} 29 \mathrm{~W} 2 \mathrm{CDCC}$ & -- & -- & -- & -. & -- & -- & -- & -. \\
\hline $36 \mathrm{~N} 29 \mathrm{~W} 4 \mathrm{CCBC}$ & -- & -- & -- & -- & -- & -- & -- & -- \\
\hline $36 \mathrm{~N} 29 \mathrm{~W} 7 \mathrm{DCC}$ & -- & -- & -- & -- & -- & -- & -- & -- \\
\hline $36 \mathrm{~N} 29 \mathrm{~W} 8 \mathrm{~A}$ & -- & -- & -- & -- & -- & -- & -- & -- \\
\hline $36 \mathrm{~N} 29 \mathrm{~W} 14 \mathrm{CDAB}$ & -- & -- & -- & -- & -- & -- & -- & -- \\
\hline $36 \mathrm{~N} 29 \mathrm{~W} 15 \mathrm{BBB}$ & -. & -. & -- & -- & -- & -- & -- & -- \\
\hline $36 \mathrm{~N} 29 \mathrm{~W} 17 \mathrm{ADBB}$ & -- & -- & -- & -- & -- & -- & -- & -- \\
\hline $36 \mathrm{~N} 29 \mathrm{~W} 19 \mathrm{BCBA}$ & -. & -. & -- & -- & -- & -- & -- & -- \\
\hline $36 \mathrm{~N} 29 \mathrm{~W} 26 \mathrm{CDC}$ & .. & -- & -- & -- & -- & -- & -- & -- \\
\hline $36 \mathrm{~N} 29 \mathrm{~W} 28 \mathrm{ADA}$ & -- & -- & -- & -- & -- & -- & -- & -- \\
\hline $36 \mathrm{~N} 29 \mathrm{~W} 29 \mathrm{ACAA}$ & -- & -- & -- & -- & -- & -- & -- & -- \\
\hline $36 \mathrm{~N} 30 \mathrm{~W} 12 \mathrm{DDCD}$ & -- & -- & - & -- & -- & -- & -- & -- \\
\hline $36 \mathrm{~N} 30 \mathrm{~W} 21 \mathrm{DAAA}$ & -- & -- & -- & -- & -- & -- & -- & -- \\
\hline $36 \mathrm{~N} 30 \mathrm{~W} 24 \mathrm{CCDB}$ & -- & -- & -- & -- & -- & - & -- & -- \\
\hline 36N30W36DDDA & -- & -- & -- & -- & -- & -- & -- & -- \\
\hline $36 \mathrm{~N} 31 \mathrm{~W} 34 \mathrm{DBBC}$ & -- & -- & -- & -. & -- & -- & -- & -- \\
\hline 37N25W 7DAAD & -- & -- & -- & -- & -- & -- & -- & -- \\
\hline $37 \mathrm{~N} 28 \mathrm{~W} 16 \mathrm{CCDD}$ & 7 & 98 & $<1$ & 1 & 3 & $<1$ & $<1$ & $<0.1$ \\
\hline $37 \mathrm{~N} 28 \mathrm{~W} 34 \mathrm{BDA} 2$ & -. & -- & -- & -- & -- & -- & -- & -- \\
\hline $37 \mathrm{~N} 29 \mathrm{~W} 12 \mathrm{CCD}$ & -- & -- & -- & -- & -- & -- & -. & -- \\
\hline $37 \mathrm{~N} 29 \mathrm{~W} 21 \mathrm{DDAD}$ & 1 & 360 & $<1$ & $<1$ & 3 & 1 & $<1$ & $<0.1$ \\
\hline 37N29W27DACD & 4 & 120 & $<1$ & $<1$ & 8 & $<1$ & $<1$ & $<0.1$ \\
\hline $37 \mathrm{~N} 29 \mathrm{~W} 34 \mathrm{ACA}$ & 1 & 270 & $<1$ & 1 & 10 & $<1$ & 1 & $<0.1$ \\
\hline
\end{tabular}


Table 2. Physical properties and concentrations of inorganic and organic constituents from reconnaissance sampling of the Ogallala aquifer-Continued

\begin{tabular}{|c|c|c|c|c|c|c|c|c|c|}
\hline Local number & $\begin{array}{c}\text { Selenium, } \\
\text { dissolved } \\
(\mu g / L \\
\text { as Se) } \\
(01145)\end{array}$ & 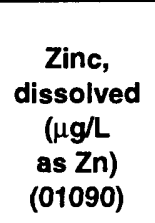 & $\begin{array}{c}\text { Alachlor, } \\
\text { total, } \\
\text { recover } \\
(\mu g / L) \\
(77825)\end{array}$ & $\begin{array}{c}\text { Ametryne, } \\
\text { total } \\
(\mu \mathrm{g} / \mathrm{L}) \\
(\mathbf{8 2 1 8 4})\end{array}$ & $\begin{array}{c}\text { Atrazine, } \\
\text { unfitrd, } \\
\text { recover } \\
(\mu g / L) \\
(39630)\end{array}$ & $\begin{array}{c}\text { Cyana- } \\
\text { zine, } \\
\text { total } \\
(\mu g / L) \\
(81757)\end{array}$ & $\begin{array}{c}\text { Dicamba } \\
\text { (Mediben) } \\
\text { (Banvel D), } \\
\text { total } \\
(\mu \mathrm{g} / \mathrm{L}) \\
(\mathbf{8 2 0 5 2})\end{array}$ & $\begin{array}{c}2,4,5-T, \\
\text { total } \\
(\mu g / L) \\
(39740)\end{array}$ & $\begin{array}{c}\text { 2,4-D, } \\
\text { total } \\
(\mu g / L) \\
(39730)\end{array}$ \\
\hline $35 \mathrm{~N} 27 \mathrm{~W} 14 \mathrm{BAAB}$ & -- & -- & -- & -- & -- & -- & -- & -- & -- \\
\hline 35N29W 2DDDD & -- & -- & -- & -- & -- & -- & -- & -- & -- \\
\hline 35N30W 6DDDD & -- & -- & -- & -- & -- & -- & -- & -- & -- \\
\hline $36 \mathrm{~N} 25 \mathrm{~W} 10 \mathrm{BABB}$ & -- & -- & -- & -- & -- & -- & -- & -- & -- \\
\hline $36 \mathrm{~N} 26 \mathrm{~W} 34 \mathrm{CCC}$ & -- & -- & -- & -- & -- & -- & -- & -- & -- \\
\hline 36N27W 1BDDD & -- & -- & -- & -- & -- & -- & -- & -- & -- \\
\hline 36N29W 2CDCC & -- & -- & -- & -. & -- & -- & -- & -- & -- \\
\hline $36 \mathrm{~N} 29 \mathrm{~W} 4 \mathrm{CCBC}$ & -- & -- & -- & -- & -. & - & -- & -- & -- \\
\hline $36 \mathrm{~N} 29 \mathrm{~W} 7 \mathrm{DCC}$ & -- & -- & $<0.10$ & $<0.10$ & $<0.1$ & $<0.10$ & $<0.01$ & $<0.01$ & $<0.01$ \\
\hline $36 \mathrm{~N} 29 \mathrm{~W} 8 \mathrm{~A}$ & -- & -- & -- & -- & -- & -- & -- & -- & -- \\
\hline $36 \mathrm{~N} 29 \mathrm{~W} 14 \mathrm{CDAB}$ & -- & -- & $<0.10$ & $<0.10$ & $<0.1$ & $<0.10$ & $<0.01$ & $<0.01$ & $<0.01$ \\
\hline $36 \mathrm{~N} 29 \mathrm{~W} 15 \mathrm{BBB}$ & -- & -- & -- & -- & -- & -- & -- & -- & -- \\
\hline 36N29W17ADBB & -- & -- & -- & -- & -- & -- & -- & -- & -- \\
\hline 36N29W19BCBA & -- & -- & -- & -- & -- & -- & -- & -- & -- \\
\hline $36 \mathrm{~N} 29 \mathrm{~W} 26 \mathrm{CDC}$ & -- & -- & -- & -- & -- & -- & - & -- & -- \\
\hline $36 \mathrm{~N} 29 \mathrm{~W} 28 \mathrm{ADA}$ & -- & -- & -- & -- & -- & -- & - & -- & -- \\
\hline 36N29W29ACAA & -- & -- & -- & -- & -- & -- & -- & -- & -- \\
\hline 36N30W12DDCD & -- & -- & -- & -- & -- & -- & -- & -- & -- \\
\hline 36N30W21DAAA & -- & -- & -- & -- & -- & - & -. & -- & -- \\
\hline $36 \mathrm{~N} 30 \mathrm{~W} 24 \mathrm{CCDB}$ & - & -- & -- & - & - & -- & -- & -- & -- \\
\hline 36N30W36DDDA & -- & - & -- & -- & -- & -- & -- & -- & -- \\
\hline 36N31W34DBBC & -- & -- & -- & -- & -- & -- & - & -- & -- \\
\hline 37N25W 7DAAD & -- & -- & -- & -- & -- & -- & -- & -- & -- \\
\hline 37N28W16CCDD & $<1$ & 12 & $<0.10$ & $<0.10$ & $<0.1$ & $<0.10$ & $<0.01$ & $<0.01$ & 0.07 \\
\hline 37N28W34BDA2 & - & -- & $<0.10$ & $<0.10$ & $<0.1$ & $<0.10$ & $<0.01$ & $<0.01$ & $<0.01$ \\
\hline $37 \mathrm{~N} 29 \mathrm{~W} 12 \mathrm{CCD}$ & -- & -- & -- & -- & -- & - & -- & -- & -- \\
\hline 37N29W21DDAD & 1 & 19 & $<0.10$ & $<0.10$ & $<0.1$ & $<0.10$ & $<0.01$ & $<0.01$ & $<0.01$ \\
\hline 37N29W27DACD & $<1$ & 20 & $<0.10$ & $<0.10$ & $<0.1$ & $<0.10$ & $<0.01$ & $<0.01$ & $<0.01$ \\
\hline $37 \mathrm{~N} 29 \mathrm{~W} 34 \mathrm{ACA}$ & 1 & 54 & $<0.10$ & $<0.10$ & $<0.1$ & $<0.10$ & $<0.01$ & $<0.01$ & 0.05 \\
\hline
\end{tabular}


Table 2. Physical properties and concentrations of inorganic and organic constituents from reconnaissance sampling of the Ogallala aquifer-Continued

\begin{tabular}{|c|c|c|c|c|c|c|c|c|}
\hline Local number & $\begin{array}{c}\text { 2,4-DP, } \\
\text { total } \\
(\mu g / L) \\
(82183)\end{array}$ & $\begin{array}{c}\text { Picloram } \\
\text { (Tordon) } \\
\text { (Amdon), } \\
\text { total } \\
\left(\mu g^{\prime} L\right) \\
(39720)\end{array}$ & $\begin{array}{c}\text { Prome- } \\
\text { tone, } \\
\text { total } \\
(\mu g / L) \\
(39056)\end{array}$ & $\begin{array}{c}\text { Prome- } \\
\text { tryne, } \\
\text { total } \\
(\mu g / L) \\
(39057)\end{array}$ & $\begin{array}{c}\text { Propazine, } \\
\text { total } \\
(\mu \mathrm{g} / \mathrm{L}) \\
(39024)\end{array}$ & $\begin{array}{c}\text { Silvex, } \\
\text { total } \\
(\mu g / L) \\
(39760)\end{array}$ & $\begin{array}{c}\text { Simazine, } \\
\text { total } \\
(\mu \mathrm{g} / \mathrm{L}) \\
(39055)\end{array}$ & $\begin{array}{c}\text { Simetryne, } \\
\text { total } \\
(\mu g / L) \\
(39054)\end{array}$ \\
\hline 35N27W14BAAB & -- & -- & -- & -- & -- & -- & -- & -- \\
\hline 35N29W 2DDDD & -- & -- & -- & -- & -- & -- & -- & -- \\
\hline 35N30W 6DDDD & -- & -- & -- & -- & -- & -- & -- & -- \\
\hline $36 \mathrm{~N} 25 \mathrm{~W} 10 \mathrm{BABB}$ & -- & -- & -- & -- & -- & -- & -- & -- \\
\hline $36 \mathrm{~N} 26 \mathrm{~W} 34 \mathrm{CCC}$ & -- & -- & -- & -- & -- & -- & -- & -- \\
\hline 36N27W 1BDDD & -- & -- & -- & -- & -- & -- & -- & -- \\
\hline $36 \mathrm{~N} 29 \mathrm{~W} 2 \mathrm{CDCC}$ & -- & -- & -- & -- & -- & -- & -- & -- \\
\hline $36 \mathrm{~N} 29 \mathrm{~W} 4 \mathrm{CCBC}$ & -- & -- & -- & -- & -- & -- & -- & -- \\
\hline 36N29W 7DCC & $<0.01$ & $<0.01$ & $<0.10$ & $<0.10$ & $<0.10$ & $<0.01$ & $<0.10$ & $<0.10$ \\
\hline $36 \mathrm{~N} 29 \mathrm{~W} 8 \mathrm{~A}$ & -- & -- & -- & -- & -- & -- & -- & -- \\
\hline 36N29W14CDAB & $<0.01$ & $<0.01$ & $<0.10$ & $<0.10$ & $<0.10$ & $<0.01$ & $<0.10$ & $<0.10$ \\
\hline $36 \mathrm{~N} 29 \mathrm{~W} 15 \mathrm{BBB}$ & -- & -- & -- & -- & -- & -- & -- & -- \\
\hline 36N29W17ADBB & -- & -- & -- & -- & -- & -- & -- & -- \\
\hline 36N29W19BCBA & -- & -- & -- & - & -- & -- & -- & -- \\
\hline $36 \mathrm{~N} 29 \mathrm{~W} 26 \mathrm{CDC}$ & -- & - & -- & -- & -- & -- & -- & -- \\
\hline 36N29W28ADA & -- & -- & -- & -- & -- & -- & -- & -- \\
\hline 36N29W29ACAA & -- & -- & -- & -- & -- & -- & -- & -- \\
\hline 36N30W12DDCD & -- & -- & -- & -- & -- & -- & -- & -- \\
\hline 36N30W21DAAA & -- & -- & -- & -- & -- & -- & -- & -- \\
\hline $36 \mathrm{~N} 30 \mathrm{~W} 24 \mathrm{CCDB}$ & -- & -- & -- & -- & -- & -- & -- & -- \\
\hline 36N30W36DDDA & -- & -- & -- & -- & -- & -- & -- & -- \\
\hline $36 \mathrm{~N} 31 \mathrm{~W} 34 \mathrm{DBBC}$ & -- & -- & -- & -- & -- & -- & -- & -- \\
\hline 37N25W 7DAAD & - & -- & -- & -- & -- & -- & -- & -- \\
\hline $37 \mathrm{~N} 28 \mathrm{~W} 16 \mathrm{CCDD}$ & $<0.01$ & $<0.01$ & $<0.10$ & $<0.10$ & $<0.10$ & $<0.01$ & $<0.10$ & $<0.10$ \\
\hline 37N28W34BDA2 & $<0.01$ & $<0.01$ & $<0.10$ & $<0.10$ & $<0.10$ & $<0.01$ & $<0.10$ & $<0.10$ \\
\hline $37 \mathrm{~N} 29 \mathrm{~W} 12 \mathrm{CCD}$ &.- & -- & -- & -- & -- & -- & -. & -- \\
\hline 37N29W21DDAD & $<0.01$ & $<0.01$ & $<0.10$ & $<0.10$ & $<0.10$ & $<0.01$ & $<0.10$ & $<0.10$ \\
\hline 37N29W27DACD & $<0.01$ & $<0.01$ & $<0.10$ & $<0.10$ & $<0.10$ & $<0.01$ & $<0.10$ & $<0.10$ \\
\hline 37N29W34ACA & $<0.01$ & $<0.01$ & $<0.10$ & $<0.10$ & $<0.10$ & $<0.01$ & $<0.10$ & $<0.10$ \\
\hline
\end{tabular}

${ }^{1}$ Laboratory value is given because field value was missing. 
Table 3. Physical properties and concentrations of inorganic and organic constituents from reconnaissance sampling of the Arikaree aquifer

[Agency collecting or analyzing sample: RST, Rosebud Sioux Tribe; USGS, U.S. Geological Survey. $\mu$ S/cm, microsiemens per centimeter; mg/L, milligrams per liter; $\mu \mathrm{g} / \mathrm{L}$, micrograms per liter; deg C, degrees Celsius; NTU, nephelometric turbidity units; unfltrd, unfiltered; recov, recoverable; $<$, less than; --, no data]

\begin{tabular}{|c|c|c|c|c|c|c|c|}
\hline Station number & Local number & County & Date & $\begin{array}{c}\text { Depth of } \\
\text { well } \\
\text { (feet) } \\
(72008)\end{array}$ & $\begin{array}{l}\text { Agency } \\
\text { collecting } \\
\text { sample }\end{array}$ & $\begin{array}{c}\text { Specific } \\
\text { conduct- } \\
\text { ance, } \\
\text { field } \\
(\mu S / \mathrm{cm}) \\
(00095)\end{array}$ & $\begin{array}{c}\text { pH, } \\
\text { field } \\
\text { (standard } \\
\text { units) } \\
(\mathbf{0 0 4 0 0 )}\end{array}$ \\
\hline 432600100303604 & 40N27W22BBA4 & Mellette & $07-31-90$ & 90.0 & USGS & 817 & 7.3 \\
\hline 432648101072401 & $40 \mathrm{~N} 32 \mathrm{~W} 17 \mathrm{ABB}$ & Mellette & $10-16-90$ & 100.0 & RST & 406 & 7.4 \\
\hline 432554101065601 & $40 \mathrm{~N} 32 \mathrm{~W} 21 \mathrm{BBBB}$ & Mellette & $10-02-90$ & 162.5 & RST & 514 & 7.9 \\
\hline 432654101130601 & 40N33W 9DDDA2 & Mellette & $10-02-90$ & 90.0 & RST & 604 & 7.4 \\
\hline 430105100445601 & $35 \mathrm{~N} 29 \mathrm{~W}$ 8DDD2 & Todd & $07-19-90$ & 250.0 & RST & 359 & 7.4 \\
\hline 430925100251701 & $37 \mathrm{~N} 26 \mathrm{~W} 20 \mathrm{CAAA} 3$ & Todd & $08-29-90$ & -- & RST & 389 & 7.3 \\
\hline 431149100462301 & $37 \mathrm{~N} 29 \mathrm{~W} 8 \mathrm{AADC}$ & Todd & $10-15-90$ & -- & RST & 302 & 7.7 \\
\hline 431404100255401 & 38N26W30DADD & Todd & $08-28-90$ & 50.0 & RST & 395 & 7.5 \\
\hline 431733100330901 & $38 \mathrm{~N} 27 \mathrm{~W} 6 \mathrm{DADC}$ & Todd & $08-28-90$ & 100.0 & RST & 397 & 7.6 \\
\hline 431648100521601 & 38N30W 9ABD & Todd & $08-31-90$ & -- & RST & 283 & 7.5 \\
\hline 431625101103001 & $38 \mathrm{~N} 33 \mathrm{~W} 12 \mathrm{CCDC}$ & Todd & $09-18-90$ & 150.0 & RST & 364 & 7.6 \\
\hline 431830100250201 & $39 \mathrm{~N} 26 \mathrm{~W} 32 \mathrm{DCDA}$ & Todd & $08-28-90$ & 153.0 & RST & 349 & 7.4 \\
\hline 431933101010501 & $39 \mathrm{~N} 31 \mathrm{~W} 29 \mathrm{BCB}$ & Todd & $08-31-90$ & 240.0 & RST & 405 & 8.5 \\
\hline 431342100344101 & $38 \mathrm{~N} 28 \mathrm{~W} 36 \mathrm{ABCB}$ & Todd & $08-02-90$ & 73.0 & USGS & 238 & 7.7 \\
\hline
\end{tabular}

\begin{tabular}{|c|c|c|c|c|c|c|c|}
\hline Local number & $\begin{array}{l}\text { Temperature, } \\
\text { water } \\
\text { (deg C) } \\
(00010)\end{array}$ & $\begin{array}{c}\text { Turbidity } \\
\text { (NTU) } \\
\text { (00076) }\end{array}$ & $\begin{array}{c}\text { Alkalinity, } \\
\text { lab } \\
\left(\mathrm{mg}^{2} \mathrm{~L} \text { as }\right. \\
\left.\mathrm{CaCO}_{3}\right) \\
(90410)\end{array}$ & $\begin{array}{c}\text { Calcium, } \\
\text { dissolved } \\
\text { (mg/L } \\
\text { as Ca) } \\
(00915)\end{array}$ & $\begin{array}{l}\text { Magnesium, } \\
\text { dissolved } \\
\text { (mg/L } \\
\text { as Mg) } \\
(00925)\end{array}$ & $\begin{array}{c}\begin{array}{c}\text { Sodium, } \\
\text { dissolved } \\
\text { (mg/L }\end{array} \\
\text { as } \mathrm{Na}) \\
(00930)\end{array}$ & $\begin{array}{l}\text { Sodium, } \\
\text { percent } \\
(00932)\end{array}$ \\
\hline 40N27W22BBA4 & 15.0 & 0.30 & 168 & 27 & 2.1 & 130 & 73 \\
\hline $40 \mathrm{~N} 32 \mathrm{~W} 17 \mathrm{ABB}$ & 14.5 & 0.50 & 196 & 41 & 2.8 & 37 & 39 \\
\hline $40 \mathrm{~N} 32 \mathrm{~W} 21 \mathrm{BBBB}$ & 14.0 & 6.3 & 244 & 11 & 0.59 & 110 & 86 \\
\hline 40N33W 9DDDA2 & 16.0 & 0.50 & 300 & 25 & 1.1 & 120 & 76 \\
\hline 35N29W 8DDD2 & 15.0 & 0.30 & 154 & 45 & 8.7 & 12 & 14 \\
\hline $37 \mathrm{~N} 26 \mathrm{~W} 20 \mathrm{CAAA} 3$ & 13.5 & 0.40 & 186 & 62 & 6.1 & 8.9 & 9 \\
\hline $37 \mathrm{~N} 29 \mathrm{~W} 8 \mathrm{AADC}$ & 13.5 & 0.30 & 146 & 39 & 6.1 & 12 & 16 \\
\hline 38N26W30DADD & 15.5 & 0.40 & 192 & 46 & 7.3 & 26 & 27 \\
\hline $38 \mathrm{~N} 27 \mathrm{~W} 6 \mathrm{DADC}$ & 15.0 & 0.50 & 193 & 23 & 2.6 & 59 & 61 \\
\hline $38 \mathrm{~N} 30 \mathrm{~W} 9 \mathrm{ABD}$ & 18.5 & 0.20 & 137 & 40 & 6.4 & 6.0 & 9 \\
\hline $38 \mathrm{~N} 33 \mathrm{~W} 12 \mathrm{CCDC}$ & 18.5 & 0.50 & 182 & 50 & 6.7 & 15 & 17 \\
\hline 39N26W32DCDA & 15.5 & 0.20 & 172 & 40 & 6.0 & 21 & 25 \\
\hline $39 \mathrm{~N} 31 \mathrm{~W} 29 \mathrm{BCB}$ & 15.5 & 0.50 & 192 & 4.6 & 0.19 & 87 & 90 \\
\hline $38 \mathrm{~N} 28 \mathrm{~W} 36 \mathrm{ABCB}$ & 13.5 & 1.6 & 113 & 34 & 4.9 & 4.7 & 8 \\
\hline
\end{tabular}


Table 3. Physical properties and concentrations of inorganic and organic constituents from reconnaissance sampling of the Arikaree aquifer-Continued

\begin{tabular}{|c|c|c|c|c|c|c|c|}
\hline Local number & $\begin{array}{l}\text { Sodium } \\
\text { adsorption } \\
\text { ratio } \\
(00931)\end{array}$ & $\begin{array}{c}\text { Potassium, } \\
\text { dissolved } \\
\text { (mg/L as K) } \\
\text { (00935) }\end{array}$ & $\begin{array}{c}\text { Sulfate, } \\
\text { dissolved } \\
\text { (mg/L as } \\
\left.\text { SO }_{4}\right) \\
(00945)\end{array}$ & $\begin{array}{c}\text { Chloride, } \\
\text { dissolved } \\
\text { (mg/L as Cl) } \\
(00940)\end{array}$ & $\begin{array}{c}\text { Fluoride, } \\
\text { dissolved } \\
\text { (mg/L as F) } \\
\text { (00950) }\end{array}$ & $\begin{array}{c}\text { Silica, } \\
\text { dissolved } \\
\text { (mg/L } \\
\left.\text { as } \mathrm{SiO}_{2}\right) \\
(00955)\end{array}$ & $\begin{array}{c}\text { Solids, } \\
\text { residue at } \\
180 \text { deg C, } \\
\text { dissolved } \\
(\mathrm{mg} / L) \\
(70300)\end{array}$ \\
\hline 40N27W22BBA4 & 6 & 24 & 70 & 110 & 0.50 & 36 & 468 \\
\hline $40 \mathrm{~N} 32 \mathrm{~W} 17 \mathrm{ABB}$ & 2 & 11 & 7.7 & 6.4 & $<0.10$ & 64 & 288 \\
\hline $40 \mathrm{~N} 32 \mathrm{~W} 2 \mathrm{IBBBB}$ & 9 & 7.0 & 16 & 5.7 & 0.40 & 64 & 338 \\
\hline 40N33W 9DDDA2 & 6 & 11 & 20 & 7.9 & $<0.10$ & 68 & 418 \\
\hline 35N29W 8DDD2 & 0.4 & 9.9 & 11 & 6.7 & 0.40 & 70 & 261 \\
\hline $37 \mathrm{~N} 26 \mathrm{~W} 20 \mathrm{CAAA} 3$ & 0.3 & 10 & 13 & 4.5 & 0.60 & 64 & 270 \\
\hline $37 \mathrm{~N} 29 \mathrm{~W} 8 \mathrm{AADC}$ & 0.5 & 8.2 & 6.5 & 3.3 & 0.40 & 59 & 222 \\
\hline 38N26W30DADD & 0.9 & 9.1 & 11 & 3.5 & 1.0 & 67 & 270 \\
\hline 38N27W 6DADC & 3 & 10 & 17 & 4.4 & 0.80 & 59 & 287 \\
\hline 38N30W 9ABD & 0.2 & 6.8 & 3.4 & 2.7 & 1.1 & 57 & 190 \\
\hline $38 \mathrm{~N} 33 \mathrm{~W} 12 \mathrm{CCDC}$ & 0.5 & 8.9 & 19 & 2.6 & 0.30 & 71 & 274 \\
\hline $39 \mathrm{~N} 26 \mathrm{~W} 32 \mathrm{DCDA}$ & 0.8 & 9.9 & 5.8 & 2.8 & 1.1 & 69 & 246 \\
\hline $39 \mathrm{~N} 31 \mathrm{~W} 29 \mathrm{BCB}$ & 11 & 6.3 & 9.3 & 8.8 & 0.40 & 62 & 268 \\
\hline $38 \mathrm{~N} 28 \mathrm{~W} 36 \mathrm{ABCB}$ & 0.2 & 5.4 & 3.0 & 2.0 & 0.30 & 62 & 186 \\
\hline
\end{tabular}

\begin{tabular}{|c|c|c|c|c|c|c|c|}
\hline Local number & $\begin{array}{c}\text { Nitrogen, } \\
\text { ammonia, } \\
\text { dissolved } \\
\text { (mg/L as N) } \\
(00608)\end{array}$ & $\begin{array}{c}\text { Nitrogen, } \\
\text { nitrite, } \\
\text { dissolved } \\
\text { (mg/L as N) } \\
\text { (00613) }\end{array}$ & $\begin{array}{c}\text { Nitrogen, } \\
\mathrm{NO}_{2}+\mathrm{NO}_{3} \\
\text { dissolved } \\
\text { (mg/L as } \mathrm{N}) \\
\text { (00631) }\end{array}$ & $\begin{array}{l}\text { Phosphorus, } \\
\text { ortho, } \\
\text { dissolved } \\
\text { (mg/L as P) } \\
\text { (00671) }\end{array}$ & $\begin{array}{c}\text { Arsenic, } \\
\text { dissolved } \\
(\mu \mathrm{g} / \mathrm{L} \text { as As) } \\
(01000)\end{array}$ & $\begin{array}{c}\text { Barium, } \\
\text { dissolved } \\
(\mu \mathrm{g} / \mathrm{L} \text { as Ba) } \\
(01005)\end{array}$ & $\begin{array}{c}\text { Cadmium, } \\
\text { dissolved } \\
(\mu g / L \text { as Cd) } \\
(01025)\end{array}$ \\
\hline $40 \mathrm{~N} 27 \mathrm{~W} 22 \mathrm{BBA} 4$ & -- & -- & 0.40 & - & -- & -- & -- \\
\hline 40N32W17ABB & $<0.01$ & $<0.01$ & 2.00 & 0.02 & 2 & 150 & $<1$ \\
\hline $40 \mathrm{~N} 32 \mathrm{~W} 21 \mathrm{BBBB}$ & $<0.01$ & $<0.01$ & 1.90 & 0.06 & -- & -- & -- \\
\hline 40N33W 9DDDA2 & $<0.01$ & $<0.01$ & 0.30 & $<0.01$ & 3 & 39 & $<1$ \\
\hline 35N29W 8DDD2 & -- & -- & 3.40 & -- & -- & -- & -- \\
\hline $37 \mathrm{~N} 26 \mathrm{~W} 20 \mathrm{CAAA} 3$ & -- & .- & 2.10 & -. & -- & -- & -- \\
\hline 37N29W 8AADC & 0.02 & $<0.01$ & 1.60 & 0.01 & 6 & 140 & $<1$ \\
\hline 38N26W30DADD & -- & -. & 1.50 & -- & -. & -- & -- \\
\hline 38N27W 6DADC & -- & -. & 0.80 & -- & -- & -- & -- \\
\hline 38N30W 9ABD & -. & -- & 1.10 & -- & -- & -- & -- \\
\hline $38 \mathrm{~N} 33 \mathrm{~W} 12 \mathrm{CCDC}$ & -- & -- & 0.40 & -- & -- & -- & -- \\
\hline 39N26W32DCDA & -- & -- & 1.40 & -- & -- & -- & -- \\
\hline $39 \mathrm{~N} 31 \mathrm{~W} 29 \mathrm{BCB}$ & - & -- & 1.20 & -- & -- & -- & -- \\
\hline $38 \mathrm{~N} 28 \mathrm{~W} 36 \mathrm{ABCB}$ & -- & -- & 1.50 & -- & -- & -- & -- \\
\hline
\end{tabular}


Table 3. Physical properties and concentrations of inorganic and organic constituents from reconnaissance sampling of the Arikaree aquifer-Continued

\begin{tabular}{|c|c|c|c|c|c|c|c|}
\hline Local number & $\begin{array}{c}\text { Chromium, } \\
\text { dissolved } \\
\text { ( } \mu \mathrm{g} / \mathrm{L} \text { as } \mathrm{Cr}) \\
(01030)\end{array}$ & $\begin{array}{c}\text { Iron, } \\
\text { dissolved } \\
(\mu \mathrm{g} / \mathrm{L} \text { as Fe }) \\
(01046)\end{array}$ & $\begin{array}{c}\text { Lead, } \\
\text { dissolved } \\
\text { ( } \mu \text { g/L as Pb) } \\
\text { (01049) }\end{array}$ & $\begin{array}{c}\text { Manganese, } \\
\text { dissolved } \\
(\mu g / L \text { as } \mathrm{Mn}) \\
(01056)\end{array}$ & $\begin{array}{c}\text { Mercury, } \\
\text { dissolved } \\
(\mu g / L \text { as Hg) } \\
(71890)\end{array}$ & $\begin{array}{c}\text { Selenium, } \\
\text { dissolved } \\
(\mu \mathrm{g} / \mathrm{L} \text { as Se) } \\
(01145)\end{array}$ & $\begin{array}{c}\text { Zinc, } \\
\text { dissolved } \\
(\mu g / L \text { as } \mathrm{Zn}) \\
(01090)\end{array}$ \\
\hline 40N27W22BBA4 & -- & -- & -- & -- & -- & $-\cdot$ & -- \\
\hline 40N32W17ABB & 1 & 3 & $<1$ & $<1$ & $<0.1$ & 2 & 17 \\
\hline $40 \mathrm{~N} 32 \mathrm{~W} 21 \mathrm{BBBB}$ & -- & - & -- & -- & -- & -- & -. \\
\hline 40N33W 9DDDA2 & 1 & $<3$ & $<1$ & $<1$ & -- & 1 & 6 \\
\hline 35N29W 8DDD2 & -- & -- & -- & -- & -- & -. & -- \\
\hline 37N26W20CAAA3 & -- & -- & -- & -- & -- & -- & -- \\
\hline 37N29W 8AADC & 2 & 3 & $<1$ & $<1$ & 0.1 & $<1$ & 5 \\
\hline 38N26W30DADD & - & -- & - & -- & -- & -- & -- \\
\hline $38 \mathrm{~N} 27 \mathrm{~W}$ 6DADC & -- & -- & -- & -- & -- & -- & -- \\
\hline 38N30W 9ABD & -- & -- & -- & -- & -- & -- & -- \\
\hline $38 \mathrm{~N} 33 \mathrm{~W} 12 \mathrm{CCDC}$ & -- & -- & -- & -- & -- & -- & -- \\
\hline 39N26W32DCDA & -- & -- & -- & -- & -- & -- & -- \\
\hline $39 \mathrm{~N} 31 \mathrm{~W} 29 \mathrm{BCB}$ & -- & -- & - & -- & -- & -- & -- \\
\hline $38 \mathrm{~N} 28 \mathrm{~W} 36 \mathrm{ABCB}$ & -- & -- & -- & -- & -- & -- & -- \\
\hline
\end{tabular}

\begin{tabular}{|c|c|c|c|c|c|c|c|}
\hline Local number & $\begin{array}{c}\text { Alachlor, } \\
\text { total, } \\
\text { recover } \\
(\mu \mathrm{g} / \mathrm{L}) \\
(77825)\end{array}$ & $\begin{array}{c}\text { Ametryne, } \\
\text { total } \\
(\mu g / L) \\
(82184)\end{array}$ & $\begin{array}{c}\text { Atrazine, } \\
\text { unfltrd, } \\
\text { recover } \\
(\mu g / L) \\
(39630)\end{array}$ & $\begin{array}{c}\text { Cyanazine, } \\
\text { total } \\
(\mu \mathrm{g} / \mathrm{L}) \\
(\mathbf{8 1 7 5 7 )}\end{array}$ & $\begin{array}{c}\text { Dicamba } \\
\text { (Mediben) } \\
\text { (Banvel D), } \\
\text { total } \\
(\mu \mathrm{g} / \mathrm{L}) \\
(82052)\end{array}$ & $\begin{array}{c}2,4,5-T, \\
\text { total } \\
(\mu g / L) \\
(39740)\end{array}$ & $\begin{array}{c}2,4-D \\
\text { total } \\
(\mu g / L) \\
(39730)\end{array}$ \\
\hline 40N27W22BBA4 & -- & -- & -- & -- & -- & -- & -- \\
\hline 40N32W17ABB & $<0.10$ & $<0.10$ & $<0.1$ & $<0.10$ & $<0.01$ & $<0.01$ & $<0.01$ \\
\hline $40 \mathrm{~N} 32 \mathrm{~W} 21 \mathrm{BBBB}$ & -- & -- & -- & -- & -- & -- & -- \\
\hline 40N33W 9DDDA2 & -- & -- & -- & -. & -- & -- & -- \\
\hline 35N29W 8DDD2 & -- & $-\cdot$ & -- & -- & -- & -- & -- \\
\hline 37N26W20CAAA3 & -- & -- & -- & -- & -- & -- & -- \\
\hline 37N29W 8AADC & $<0.10$ & $<0.10$ & $<0.1$ & $<0.10$ & $<0.01$ & $<0.01$ & 0.04 \\
\hline 38N26W30DADD & -- & -- & -- & -- & -- & -- & -- \\
\hline 38N27W 6DADC & -- & -- & -- & -- & -- & -- & -- \\
\hline 38N30W 9ABD & -- & -- & -- & -- & -- & - & -- \\
\hline $38 \mathrm{~N} 33 \mathrm{~W} 12 \mathrm{CCDC}$ & -- & -- & -- & -- & -- & -- & -- \\
\hline 39N26W32DCDA & -- & -- & -- & -- & -. & -- & -- \\
\hline 39N31W29BCB & -- & -- & -- & -- & -- & -- & -. \\
\hline $38 \mathrm{~N} 28 \mathrm{~W} 36 \mathrm{ABCB}$ & -- & -- & -- & -- & -- & -. & -- \\
\hline
\end{tabular}


Table 3. Physical properties and concentrations of inorganic and organic constituents from reconnaissance sampling of the Arikaree aquifer-Continued

\begin{tabular}{|c|c|c|c|c|c|c|c|c|}
\hline Local number & $\begin{array}{c}\text { 2,4-DP, } \\
\text { total } \\
(\mu \mathrm{g} / \mathrm{L}) \\
(82183)\end{array}$ & $\begin{array}{c}\text { Picloram } \\
\text { (Tordon) } \\
\text { (Amdon), } \\
\text { total } \\
(\mu \mathrm{g} / \mathrm{L}) \\
(39720)\end{array}$ & $\begin{array}{c}\text { Prome- } \\
\text { tone, } \\
\text { total } \\
(\mu g / L) \\
(39056)\end{array}$ & $\begin{array}{c}\text { Prome- } \\
\text { tryne, } \\
\text { total } \\
(\mu g / L) \\
(39057)\end{array}$ & $\begin{array}{c}\text { Propazine, } \\
\text { total } \\
(\mu g / L) \\
(39024)\end{array}$ & $\begin{array}{c}\text { Silvex, } \\
\text { total } \\
(\mu \mathrm{g} / \mathrm{L}) \\
(39760)\end{array}$ & $\begin{array}{c}\text { Simazine, } \\
\text { total } \\
(\mu g / L) \\
(39055)\end{array}$ & $\begin{array}{c}\text { Simetryne, } \\
\text { total } \\
(\mu g / L) \\
(39054)\end{array}$ \\
\hline 40N27W22BBA4 & -- & - & -- & -- & -- & -- & -- & -- \\
\hline $40 \mathrm{~N} 32 \mathrm{~W} 17 \mathrm{ABB}$ & $<0.01$ & $<0.01$ & $<0.10$ & $<0.10$ & $<0.10$ & $<0.01$ & $<0.10$ & $<0.10$ \\
\hline $40 \mathrm{~N} 32 \mathrm{~W} 21 \mathrm{BBBB}$ & -- & -- & -- & -- & -- & -- & -- & -- \\
\hline 40N33W 9DDDA2 & -- & -- & -- & -- & -- & -- & -- & -- \\
\hline 35N29W 8DDD2 & -- & -- & -- & -- & -- & -- & -- & -- \\
\hline 37N26W20CAAA3 & -- & -- & -- & -- & -- & -- & -- & -- \\
\hline $37 \mathrm{~N} 29 \mathrm{~W} 8 \mathrm{AADC}$ & $<0.01$ & $<0.01$ & $<0.10$ & $<0.10$ & $<0.10$ & $<0.01$ & $<0.10$ & $<0.10$ \\
\hline 38N26W30DADD & -- & -- & -- & -- & -- & -- & -- & -- \\
\hline $38 \mathrm{~N} 27 \mathrm{~W} 6 \mathrm{DADC}$ & -- & -- & -- & -- & -- & -- & -- & -- \\
\hline 38N30W 9ABD & -- & -- & -- & -- & -- & -- & -- & -- \\
\hline $38 \mathrm{~N} 33 \mathrm{~W} 12 \mathrm{CCDC}$ & -- & -- & -- & -- & -- & -- & -- & -- \\
\hline 39N26W32DCDA & -- & -- & -- & -- & -- & -- & -- & -- \\
\hline $39 \mathrm{~N} 31 \mathrm{~W} 29 \mathrm{BCB}$ & -- & -- & -- & -- & -- & -- & -- & -- \\
\hline $38 \mathrm{~N} 28 \mathrm{~W} 36 \mathrm{ABCB}$ & -- & -- & -- & -- & -- & -- & -- & -- \\
\hline
\end{tabular}


Table 4. Physical properties and concentrations of inorganic constituents from reconnaissance sampling of the White River aquifer

[Agency collecting or analyzing sample: RST, Rosebud Sioux Tribe. $\mu \mathrm{S} / \mathrm{cm}$, microsiemens per centimeter; mg/L, milligrams per liter; $\mu \mathrm{g} / \mathrm{L}$, micrograms per liter; deg C, degrees Celsius; NTU, nephelometric turbidity units; <, less than; --, no data]

\begin{tabular}{|c|c|c|c|c|c|c|c|}
\hline Station number & Local number & County & Date & $\begin{array}{c}\text { Depth of } \\
\text { well } \\
\text { (feet) } \\
\text { (72008) }\end{array}$ & $\begin{array}{l}\text { Agency } \\
\text { collecting } \\
\text { sample }\end{array}$ & $\begin{array}{c}\text { Specific } \\
\text { conduct- } \\
\text { ance, } \\
\text { field } \\
(\mu S / \mathrm{cm}) \\
(00095)\end{array}$ & $\begin{array}{c}\text { pH, } \\
\text { field } \\
\text { (standard } \\
\text { units) } \\
(00400)\end{array}$ \\
\hline 432558100513303 & $40 \mathrm{~N} 30 \mathrm{~W} 22 \mathrm{BAB}$ & Mellette & $10-16-90$ & 60.0 & RST & 480 & 7.3 \\
\hline 432728100562703 & $40 \mathrm{~N} 31 \mathrm{~W} 12 \mathrm{BDB} 3$ & Mellette & $10-16-90$ & 40.0 & RST & 910 & 7.1 \\
\hline 432440100575501 & $40 \mathrm{~N} 31 \mathrm{~W} 28 \mathrm{CAA}$ & Mellette & $10-16-90$ & -- & RST & 896 & 6.9 \\
\hline 432832101105201 & $40 \mathrm{~N} 33 \mathrm{~W} 2 \mathrm{AAB}$ & Mellette & $10-02-90$ & 110.0 & RST & 567 & 7.1 \\
\hline 433206100374301 & $41 \mathrm{~N} 28 \mathrm{~W} 14 \mathrm{BBB}$ & Mellette & $08-14-90$ & 47.0 & RST & 820 & 6.9 \\
\hline 433055101004902 & 41N31W21BDB2 & Mellette & $10-02-90$ & 40.0 & RST & 1,040 & 6.8 \\
\hline 433506100351401 & $42 \mathrm{~N} 27 \mathrm{~W} 30 \mathrm{CBAA}$ & Mellette & $08-14-90$ & 70.0 & RST & ${ }^{1} 2,890$ & 6.7 \\
\hline 433907101070601 & $43 \mathrm{~N} 32 \mathrm{~W} 34 \mathrm{CCC}$ & Mellette & $09-05-90$ & 8.0 & RST & 708 & 7.1 \\
\hline 431314100130501 & 38N25W36CACD & Todd & $08-29-90$ & 90.0 & RST & 296 & 7.5 \\
\hline 431902100174201 & $39 \mathrm{~N} 25 \mathrm{~W} 33 \mathrm{BBAA}$ & Todd & $08-28-90$ & 30.0 & RST & 651 & 7.0 \\
\hline
\end{tabular}

\begin{tabular}{|c|c|c|c|c|c|c|c|}
\hline Local number & $\begin{array}{l}\text { Temperature, } \\
\text { water } \\
\text { (deg C) } \\
(00010)\end{array}$ & $\begin{array}{c}\text { Turbidity } \\
\text { (NTU) } \\
\text { (00076) }\end{array}$ & $\begin{array}{c}\text { Alkalinity, } \\
\text { lab } \\
\text { (mg/L as } \\
\left.\mathrm{CaCO}_{3}\right) \\
(90410)\end{array}$ & $\begin{array}{c}\text { Calcium, } \\
\text { dissolved } \\
\text { (mg/L } \\
\text { as Ca) } \\
(00915)\end{array}$ & $\begin{array}{c}\text { Magnesium, } \\
\text { dissolved } \\
\text { (mg/L } \\
\text { as Mg) } \\
(00925)\end{array}$ & $\begin{array}{c}\begin{array}{c}\text { Sodium, } \\
\text { dissolved } \\
\text { (mg/L }\end{array} \\
\text { as } \mathrm{Na}) \\
(00930)\end{array}$ & $\begin{array}{l}\text { Sodium, } \\
\text { percent } \\
\text { (00932) }\end{array}$ \\
\hline 40N30W22BAB & 16.0 & 0.40 & 213 & 58 & 6.1 & 29 & 26 \\
\hline $40 \mathrm{~N} 31 \mathrm{~W} 12 \mathrm{BDB} 3$ & 16.5 & 0.40 & 422 & 44 & 2.5 & 160 & 72 \\
\hline $40 \mathrm{~N} 31 \mathrm{~W} 28 \mathrm{CAA}$ & 11.0 & 0.30 & 469 & 68 & 3.3 & 130 & 59 \\
\hline $40 \mathrm{~N} 33 \mathrm{~W} 2 \mathrm{AAB}$ & 13.5 & 0.40 & 288 & 66 & 3.0 & 58 & 40 \\
\hline $41 \mathrm{~N} 28 \mathrm{~W} 14 \mathrm{BBB}$ & 20.0 & 0.40 & 421 & 86 & 8.6 & 87 & 42 \\
\hline $41 \mathrm{~N} 31 \mathrm{~W} 21 \mathrm{BDB} 2$ & 15.5 & 17 & 589 & 95 & 7.7 & 130 & 50 \\
\hline $42 \mathrm{~N} 27 \mathrm{~W} 30 \mathrm{CBAA}$ & 17.5 & 0.40 & 396 & 360 & 55 & 290 & 35 \\
\hline $43 \mathrm{~N} 32 \mathrm{~W} 34 \mathrm{CCC}$ & 20.0 & 0.70 & 387 & 46 & 2.4 & 120 & 67 \\
\hline $38 \mathrm{~N} 25 \mathrm{~W} 36 \mathrm{CACD}$ & 16.5 & 0.40 & 137 & 44 & 7.1 & 5.1 & 7 \\
\hline $39 \mathrm{~N} 25 \mathrm{~W} 33 \mathrm{BBAA}$ & 18.5 & 0.40 & 211 & 45 & 5.6 & 83 & 54 \\
\hline
\end{tabular}


Table 4. Physical properties and concentrations of inorganic constituents from reconnaissance sampling of the White River aquifer-Continued

\begin{tabular}{|c|c|c|c|c|c|c|c|}
\hline Local number & $\begin{array}{l}\text { Sodium } \\
\text { adsorption } \\
\text { ratio } \\
(00931)\end{array}$ & $\begin{array}{l}\text { Potassium, } \\
\text { dissolved } \\
\text { (mg/L } \\
\text { as K) } \\
\text { (00935) }\end{array}$ & $\begin{array}{c}\text { Sulfate, } \\
\text { dissolved } \\
\left(\mathrm{mg}^{2} \mathrm{Las} \mathrm{SO}_{4}\right) \\
(00945)\end{array}$ & $\begin{array}{l}\text { Chloride, } \\
\text { dissolved } \\
\text { (mg/L } \\
\text { as Cl) } \\
(00940)\end{array}$ & $\begin{array}{c}\text { Fluoride, } \\
\text { dissolved } \\
\text { (mg/L } \\
\text { as F) } \\
(00950)\end{array}$ & $\begin{array}{c}\text { Silica, } \\
\text { dissolved } \\
\text { (mg/L } \\
\left.\text { as } \mathrm{SiO}_{2}\right) \\
(00955)\end{array}$ & $\begin{array}{c}\text { Solids, } \\
\text { residue } \\
\text { at } 180 \text { deg C, } \\
\text { dissolved } \\
(\mathbf{m g} / \mathrm{L}) \\
(70300)\end{array}$ \\
\hline 40N30W22BAB & 1 & 11 & 16 & 6.1 & 0.30 & 48 & 299 \\
\hline 40N31 W12BDB3 & 6 & 11 & 37 & 21 & 0.20 & 47 & 575 \\
\hline $40 \mathrm{~N} 31 \mathrm{~W} 28 \mathrm{CAA}$ & 4 & 11 & 14 & 13 & 0.20 & 47 & 567 \\
\hline $40 \mathrm{~N} 33 \mathrm{~W} 2 \mathrm{AAB}$ & 2 & 9.0 & 18 & 5.3 & $<0.10$ & 65 & 383 \\
\hline $41 \mathrm{~N} 28 \mathrm{~W} 14 \mathrm{BBB}$ & 2 & 12 & 21 & 13 & 0.50 & 42 & 513 \\
\hline 41N31W21BDB2 & 3 & 8.0 & $<1.0$ & 10 & 0.10 & 48 & 674 \\
\hline $42 \mathrm{~N} 27 \mathrm{~W} 30 \mathrm{CBAA}$ & 4 & 20 & 1,300 & 16 & 0.30 & 29 & 2450 \\
\hline $43 \mathrm{~N} 32 \mathrm{~W} 34 \mathrm{CCC}$ & 5 & 5.3 & 18 & 7.6 & 0.40 & 33 & 439 \\
\hline 38N25W36CACD & 0.2 & 6.1 & 11 & 2.7 & 0.70 & 62 & 220 \\
\hline $39 \mathrm{~N} 25 \mathrm{~W} 33 \mathrm{BBAA}$ & 3 & 16 & 110 & 9.5 & 1.1 & 33 & 420 \\
\hline
\end{tabular}

\begin{tabular}{|c|c|c|c|c|c|c|c|}
\hline Local number & $\begin{array}{c}\text { Nitrogen, } \\
\text { ammonia, } \\
\text { dissolved } \\
\text { (mg/L as } \mathrm{N} \text { ) } \\
\text { (00608) }\end{array}$ & $\begin{array}{c}\text { Nitrogen, } \\
\text { nitrite, } \\
\text { dissolved } \\
\text { (mg/L as N) } \\
\text { (00613) }\end{array}$ & $\begin{array}{c}\text { Nitrogen, } \\
\mathrm{NO}_{2}+\mathrm{NO}_{3} \\
\text { dissolved } \\
\text { (mg/L as } \mathrm{N} \text { ) } \\
\text { (00631) }\end{array}$ & $\begin{array}{l}\text { Phosphorus, } \\
\text { ortho, } \\
\text { dissolved } \\
\text { (mg/L as P) } \\
\text { (00671) }\end{array}$ & $\begin{array}{c}\text { Arsenic, } \\
\text { dissolved } \\
(\mu \mathrm{g} / \mathrm{L} \text { as As) } \\
(01000)\end{array}$ & $\begin{array}{c}\text { Barium, } \\
\text { dissolved } \\
(\mu \mathrm{g} / \mathrm{L} \text { as Ba) } \\
(01005)\end{array}$ & $\begin{array}{c}\text { Cadmium, } \\
\text { dissolved } \\
(\mu g / L \text { as Cd) } \\
(01025)\end{array}$ \\
\hline 40N30W22BAB & 0.01 & $<0.01$ & 4.40 & 0.14 & 10 & 270 & $<1$ \\
\hline $40 \mathrm{~N} 31 \mathrm{~W} 12 \mathrm{BDB} 3$ & 0.02 & $<0.01$ & 2.30 & 0.03 & -- & -- & -- \\
\hline $40 \mathrm{~N} 31 \mathrm{~W} 28 \mathrm{CAA}$ & $<0.01$ & $<0.01$ & 0.60 & 0.18 & -. & -- & -- \\
\hline $40 \mathrm{~N} 33 \mathrm{~W} 2 \mathrm{AAB}$ & $<0.01$ & $<0.01$ & $<0.10$ & 0.02 & -- & -- & -- \\
\hline 41N28W14BBB & -- & -- & 0.80 & -- & -- & -- & -- \\
\hline $41 \mathrm{~N} 31 \mathrm{~W} 21 \mathrm{BDB} 2$ & 0.25 & $<0.01$ & $<0.10$ & 0.40 & -- & -- & -- \\
\hline $42 \mathrm{~N} 27 \mathrm{~W} 30 \mathrm{CBAA}$ & -- & -- & 1.60 & -- & -- & -- & -- \\
\hline $43 N 32 W 34 C C C$ & -- & -- & $<0.10$ & -- & 7 & 120 & 1 \\
\hline $38 \mathrm{~N} 25 \mathrm{~W} 36 \mathrm{CACD}$ & -- & -- & 2.90 & -- & -- & -- & -- \\
\hline 39N25W33BBAA & -- & -- & 0.20 & -- & -- & -- & -- \\
\hline
\end{tabular}


Table 4. Physical properties and concentrations of inorganic constituents from reconnaissance sampling of the White River aquifer-Continued

\begin{tabular}{|c|c|c|c|c|c|c|c|}
\hline Local number & $\begin{array}{c}\text { Chromium, } \\
\text { dissolved } \\
(\mu g / L \text { as } \mathrm{Cr}) \\
(01030)\end{array}$ & $\begin{array}{c}\text { Iron, } \\
\text { dissolved } \\
(\mu g / L \text { as Fe) } \\
(01046)\end{array}$ & $\begin{array}{c}\text { Lead, } \\
\text { dissolved } \\
(\mu \mathrm{g} / \mathrm{L} \text { as } \mathrm{Pb}) \\
(01049)\end{array}$ & $\begin{array}{c}\text { Manganese, } \\
\text { dissolved } \\
(\mu \mathrm{g} / \mathrm{L} \text { as } \mathrm{Mn}) \\
(01056)\end{array}$ & $\begin{array}{c}\text { Mercury, } \\
\text { dissolved } \\
(\mu \mathrm{g} / \mathrm{L} \text { as Hg) } \\
(71890)\end{array}$ & $\begin{array}{c}\text { Selenium, } \\
\text { dissolved } \\
(\mu \mathrm{g} / \mathrm{L} \text { as Se }) \\
(01145)\end{array}$ & $\begin{array}{c}\text { Zinc, } \\
\text { dissolved } \\
(\mu \mathrm{g} / \mathrm{L} \text { as } \mathrm{Zn}) \\
(01090)\end{array}$ \\
\hline 40N30W22BAB & 2 & 3 & $<1$ & $<1$ & $<0.1$ & 4 & 11 \\
\hline $40 \mathrm{~N} 31 \mathrm{~W} 12 \mathrm{BDB} 3$ & -- & -- & -- & -- & .- & -- & .. \\
\hline $40 \mathrm{~N} 31 \mathrm{~W} 28 \mathrm{CAA}$ & -. & -- & -- & -. & -- & -- & -- \\
\hline $40 \mathrm{~N} 33 \mathrm{~W} 2 \mathrm{AAB}$ & -- & -- & -- & -- & -- & -- & -- \\
\hline $41 N 28 W 14 B B B$ & -- & - & -- & -. & - & -- & -- \\
\hline $41 \mathrm{~N} 31 \mathrm{~W} 21 \mathrm{BDB} 2$ & -- & - & -- & -- & -- & -- & -- \\
\hline $42 \mathrm{~N} 27 \mathrm{~W} 30 \mathrm{CBAA}$ & - & -- & -- & -- & -- & -- & -- \\
\hline $43 N 32 W 34 C C C$ & $<1$ & 42 & $<1$ & 810 & $<0.1$ & $<1$ & 4 \\
\hline 38N25W36CACD & -- & -- & -- & -- & -- & -- & -- \\
\hline $39 \mathrm{~N} 25 \mathrm{~W} 33 \mathrm{BBAA}$ & -- & -- & -- & -- & -- & -- & -- \\
\hline
\end{tabular}

${ }^{1}$ Lab value is given because field value was missing. 
Table 5. Physical properties and concentrations of inorganic and organic constituents from reconnaissance sampling of the Pierre Shale aquifer

[Agency collecting or analyzing sample: RST, Rosebud Sioux Tribe. $\mu \mathrm{S} / \mathrm{cm}$, microsiemens per centimeter; $\mathrm{mg} / \mathrm{L}$, milligrams per liter; $\mu \mathrm{g} / \mathrm{L}$, micrograms per liter; deg C, degrees Celsius; NTU, nephelometric turbidity units; recover, recoverable; unfltrd, unfiltered; <, less than; --, no data]

\begin{tabular}{|c|c|c|c|c|c|c|c|c|}
\hline Station number & Local number & County & Date & $\begin{array}{c}\text { Depth of } \\
\text { well } \\
\text { (feet) } \\
(72008)\end{array}$ & $\begin{array}{l}\text { Agency } \\
\text { collecting } \\
\text { sample }\end{array}$ & $\begin{array}{c}\text { Specific } \\
\text { conduct- } \\
\text { ance, } \\
\text { field } \\
(\mu \mathrm{S} / \mathrm{cm}) \\
(00095)\end{array}$ & $\begin{array}{c}\text { pH, } \\
\text { field } \\
\text { (standard } \\
\text { units) } \\
(00400)\end{array}$ & $\begin{array}{c}\text { Tempera- } \\
\text { ture, } \\
\text { water } \\
\text { (deg C) } \\
\text { (00010) }\end{array}$ \\
\hline 432805100174501 & $40 \mathrm{~N} 25 \mathrm{~W} 5 \mathrm{ADC}$ & Mellette & $10-03-90$ & 35.0 & RST & ${ }^{1} 911$ & 17.5 & -- \\
\hline 433033100315201 & $41 \mathrm{~N} 27 \mathrm{~W} 21 \mathrm{DDD}$ & Mellette & $10-22-90$ & 21.0 & RST & 2,930 & 6.7 & 13.0 \\
\hline 433027100471601 & 41N29W20DDD2 & Mellette & $08-14-90$ & -- & RST & 1,130 & 7.1 & 14.5 \\
\hline 433229100563101 & 41N30W 7DDBD & Mellette & $10-16-90$ & 32.0 & RST & 1,310 & 7.2 & 15.5 \\
\hline 433717100221701 & $42 \mathrm{~N} 26 \mathrm{~W} 14 \mathrm{AABA}$ & Mellette & $08-16-90$ & 50.0 & RST & 1,560 & 6.8 & 12.0 \\
\hline 433427100252601 & $42 \mathrm{~N} 26 \mathrm{~W} 33 \mathrm{BDC}$ & Mellette & $08-16-90$ & 30.0 & RST & 1,840 & 7.0 & 17.0 \\
\hline 433815100325501 & $42 \mathrm{~N} 27 \mathrm{~W} 9 \mathrm{BBB}$ & Mellette & $10-22-90$ & -- & RST & 2,380 & 7.0 & 15.5 \\
\hline 433650100501701 & $42 \mathrm{~N} 30 \mathrm{~W} 13 \mathrm{ACDB}$ & Mellette & $09-06-90$ & -- & RST & 2,870 & 8.0 & 22.5 \\
\hline 433426100563602 & $42 \mathrm{~N} 30 \mathrm{~W} 31 \mathrm{ADC} 2$ & Mellette & $09-06-90$ & 24.0 & RST & 1,500 & 7.0 & 16.0 \\
\hline 433726100584501 & $42 \mathrm{~N} 31 \mathrm{~W} 11 \mathrm{CCB}$ & Mellette & $09-06-90$ & 20.0 & RST & 1,740 & 7.1 & 19.0 \\
\hline 433729101072802 & $42 \mathrm{~N} 32 \mathrm{~W} 9 \mathrm{CDB}$ & Mellette & $09-05-90$ & 250.0 & RST & 3,170 & 7.7 & 22.0 \\
\hline 433956100221702 & 43N25W35AAA & Mellette & $08-16-90$ & 35.0 & RST & 1,620 & 7.2 & 11.5 \\
\hline 433821100285201 & $43 \mathrm{~N} 27 \mathrm{~W} 36 \mathrm{CDDD}$ & Mellette & $10-22-90$ & 40.0 & RST & 711 & 7.1 & 16.0 \\
\hline 434202100422601 & $43 \mathrm{~N} 28 \mathrm{~W} 18 \mathrm{CCBC}$ & Mellette & $08-15-90$ & 65.0 & RST & 1,550 & 7.2 & 18.5 \\
\hline 434345100532701 & 43N30W 4DAD & Mellette & $09-17-90$ & 42.0 & RST & 4,260 & 6.8 & 16.5 \\
\hline 434246101014001 & $43 \mathrm{~N} 31 \mathrm{~W} 8 \mathrm{DBDC}$ & Mellette & $09-05-90$ & 68.0 & RST & 5,430 & 6.6 & 18.0 \\
\hline 434529100535003 & $44 N 30 W 28 D A C$ & Mellette & $09-17-90$ & 42.0 & RST & 6,200 & 6.7 & 15.0 \\
\hline 434652101010801 & $44 \mathrm{~N} 31 \mathrm{~W} 16 \mathrm{CCC}$ & Mellette & $09-05-90$ & 70.0 & RST & 2,680 & 7.0 & 16.5 \\
\hline 434734101054001 & $44 \mathrm{~N} 32 \mathrm{~W} 14 \mathrm{BABC}$ & Mellette & $09-05-90$ & 10.0 & RST & 658 & 7.0 & 20.0 \\
\hline
\end{tabular}


Table 5. Physical properties and concentrations of inorganic and organic constituents from reconnaissance sampling of the Pierre aquifer-Continued

\begin{tabular}{|c|c|c|c|c|c|c|c|c|c|}
\hline Local number & $\begin{array}{c}\text { Turbidity } \\
\text { (NTU) } \\
\text { (00076) }\end{array}$ & $\begin{array}{c}\text { Alkalinity, } \\
\text { lab } \\
\text { (mg/L as } \\
\left.\mathrm{CaCO}_{3}\right) \\
(90410)\end{array}$ & $\begin{array}{c}\text { Calcium, } \\
\text { dissolved } \\
\text { (mg/L } \\
\text { as Ca) } \\
(00915)\end{array}$ & $\begin{array}{l}\text { Magne- } \\
\text { sium, } \\
\text { dissolved } \\
\text { (mgll } \\
\text { as Mg) } \\
(00925)\end{array}$ & $\begin{array}{c}\text { Sodium, } \\
\text { dissolved } \\
\text { (mgl } \\
\text { as } \mathrm{Na}) \\
(00930)\end{array}$ & $\begin{array}{l}\text { Sodium, } \\
\text { percent } \\
\text { (00932) }\end{array}$ & $\begin{array}{c}\text { Sodium } \\
\text { adsorption } \\
\text { ratio } \\
\text { (00931) }\end{array}$ & $\begin{array}{c}\text { Potassium, } \\
\text { dissolved } \\
\text { (mg/L } \\
\text { as K) } \\
(00935)\end{array}$ & $\begin{array}{c}\text { Sulfate, } \\
\text { dissolved } \\
\text { (mgll } \\
\text { as SO } \\
(00945)\end{array}$ \\
\hline 40N25W 5ADC & 1.0 & 227 & 160 & 13 & 18 & 8 & 0.4 & 6.5 & 240 \\
\hline $41 \mathrm{~N} 27 \mathrm{~W} 21 \mathrm{DDD}$ & 0.50 & 526 & 490 & 54 & 140 & 17 & 2 & 24 & 1,200 \\
\hline 41N29W20DDD2 & 0.60 & 366 & 130 & 17 & 91 & 33 & 2 & 11 & 200 \\
\hline 41N30W 7DDBD & 0.40 & 330 & 74 & 13 & 200 & 63 & 6 & 9.7 & 330 \\
\hline $42 \mathrm{~N} 26 \mathrm{~W} 14 \mathrm{AABA}$ & 1.6 & 349 & 200 & 46 & .82 & 20 & 1 & 11 & 530 \\
\hline $42 \mathrm{~N} 26 \mathrm{~W} 33 \mathrm{BDC}$ & 78 & 122 & 280 & 51 & 41 & 9 & 0.6 & 33 & 780 \\
\hline $42 \mathrm{~N} 27 \mathrm{~W} 9 \mathrm{BBB}$ & 0.40 & 244 & 350 & 64 & 110 & 17 & 1 & 24 & 1,100 \\
\hline $42 \mathrm{~N} 30 \mathrm{~W} 13 \mathrm{ACDB}$ & 5.5 & 405 & 7.6 & 1.4 & 570 & 98 & 50 & 5.4 & 930 \\
\hline 42N30W31ADC2 & 4.0 & 329 & 130 & 23 & 160 & 44 & 3 & 19 & 430 \\
\hline $42 \mathrm{~N} 31 \mathrm{~W} 11 \mathrm{CCB}$ & 0.50 & 326 & 160 & 28 & 200 & 45 & 4 & 8.8 & 600 \\
\hline $42 \mathrm{~N} 32 \mathrm{~W} 9 \mathrm{CDB}$ & 0.80 & 191 & 26 & 0.90 & 580 & 94 & 30 & 4.3 & $<1.0$ \\
\hline $43 \mathrm{~N} 25 \mathrm{~W} 35 \mathrm{AAA}$ & 0.20 & 282 & 130 & 27 & 180 & 47 & 4 & 10 & 470 \\
\hline $43 N 27 W 36 C D D D$ & 0.40 & 241 & 110 & 13 & 13 & 8 & 0.3 & 8.7 & 110 \\
\hline $43 \mathrm{~N} 28 \mathrm{~W} 18 \mathrm{CCBC}$ & 0.30 & 320 & 82 & 18 & 250 & 65 & 7 & 12 & 420 \\
\hline 43N30W 4DAD & 0.50 & 454 & 470 & 130 & 430 & 35 & 5 & 28 & 1,800 \\
\hline $43 N 31 \mathrm{~W} 8 \mathrm{DBDC}$ & 1.5 & 549 & 390 & 170 & 770 & 49 & 8 & 33 & 2,800 \\
\hline $44 \mathrm{~N} 30 \mathrm{~W} 28 \mathrm{DAC}$ & 1.0 & 550 & 430 & 280 & 830 & 45 & 8 & 18 & 3,000 \\
\hline $44 N 31 W 16 C C C$ & 1.3 & 298 & 160 & 34 & 390 & 60 & 7 & 25 & 920 \\
\hline $44 \mathrm{~N} 32 \mathrm{~W} 14 \mathrm{BABC}$ & 0.30 & 257 & 100 & 10 & 14 & 9 & 0.3 & 12 & 29 \\
\hline
\end{tabular}


Table 5. Physical properties and concentrations of inorganic and organic constituents from reconnaissance sampling of the Pierre aquifer-Continued

\begin{tabular}{|c|c|c|c|c|c|c|c|c|}
\hline Local number & $\begin{array}{c}\text { Chloride, } \\
\text { dissolved } \\
\text { (mgll } \\
\text { as Cl) } \\
(00940)\end{array}$ & $\begin{array}{c}\text { Fluoride, } \\
\text { dissolved } \\
\text { (mg/L } \\
\text { as F) } \\
(00950)\end{array}$ & $\begin{array}{c}\text { Silica, } \\
\text { dissolved } \\
\text { (mg/L } \\
\left.\text { as } \mathrm{SiO}_{2}\right) \\
(00955)\end{array}$ & $\begin{array}{c}\text { Solids, } \\
\text { residue at } \\
180 \text { deg C, } \\
\text { dissolved } \\
\text { (mg/L) } \\
(70300)\end{array}$ & $\begin{array}{c}\text { Nitrogen, } \\
\text { ammonia, } \\
\text { dissolved } \\
\text { (mg/L } \\
\text { as } N) \\
(00608)\end{array}$ & $\begin{array}{c}\text { Nitrogen, } \\
\text { nitrite, } \\
\text { dissolved } \\
\text { (mg/L } \\
\text { as } \mathrm{N} \text { ) } \\
(00613)\end{array}$ & $\begin{array}{c}\text { Nitrogen, } \\
\mathrm{NO}_{2}+\mathrm{NO}_{3} \\
\text { dissolved } \\
\text { (mg/ } \\
\text { as } \mathrm{N}) \\
(00631)\end{array}$ & $\begin{array}{c}\text { Phos- } \\
\text { phorus, } \\
\text { ortho, } \\
\text { dissolved } \\
\text { (mg/L } \\
\text { as P) } \\
(00671)\end{array}$ \\
\hline $40 \mathrm{~N} 25 \mathrm{~W} 5 \mathrm{ADC}$ & 18 & $<0.10$ & 31 & 650 & $<0.01$ & $<0.01$ & 3.10 & 0.07 \\
\hline $41 \mathrm{~N} 27 \mathrm{~W} 21 \mathrm{DDD}$ & 83 & 0.30 & 34 & 2,600 & 0.04 & $<0.01$ & 22.0 & 0.02 \\
\hline 41N29W20DDD2 & 23 & 0.40 & 37 & 726 & -- & -- & 4.30 & -- \\
\hline 41N30W 7DDBD & 7.5 & 0.20 & 27 & 872 & 0.01 & $<0.01$ & 1.00 & 0.01 \\
\hline 42N26W14AABA & 25 & 0.50 & 17 & 1,150 & -- & -- & 0.20 & -- \\
\hline 42N26W33BDC & 77 & 2.4 & 28 & 1,480 & -. & -- & $<0.10$ & -- \\
\hline $42 N 27 W$ 9BBB & 51 & 0.30 & 35 & 1,940 & 0.03 & $<0.01$ & 1.20 & 0.01 \\
\hline 42N30W13ACDB & 35 & 2.5 & 25 & 1,920 & -- & -- & $<0.10$ & -- \\
\hline 42N30W31ADC2 & 26 & 0.70 & 19 & 1,030 & -- & -- & $<0.10$ & -- \\
\hline $42 \mathrm{~N} 31 \mathrm{~W} 11 \mathrm{CCB}$ & 5.6 & 0.60 & 28 & 1,280 & -- & -- & $<0.10$ & -- \\
\hline $42 \mathrm{~N} 32 \mathrm{~W} 9 \mathrm{CDB}$ & 790 & 0.60 & 11 & 1,640 & -- & -- & $<0.10$ & -- \\
\hline $43 \mathrm{~N} 25 \mathrm{~W} 35 \mathrm{AAA}$ & 67 & $<0.10$ & 14 & 1,100 & -- & -- & $<0.10$ & -- \\
\hline 43N27W36CDDD & 11 & 0.20 & 45 & 490 & 0.01 & $<0.01$ & 3.10 & 0.12 \\
\hline $43 \mathrm{~N} 28 \mathrm{~W} 18 \mathrm{CCBC}$ & 32 & 0.30 & 12 & 1,040 & -- & -- & 0.20 & -- \\
\hline $43 N 30 W$ 4DAD & 93 & 0.40 & 12 & 3,750 & -- & -- & 1.70 & -- \\
\hline $43 \mathrm{~N} 31 \mathrm{~W} 8 \mathrm{DBDC}$ & 59 & 0.50 & 13 & 4,350 & -- & -- & 1.70 & -- \\
\hline $44 \mathrm{~N} 30 \mathrm{~W} 28 \mathrm{DAC}$ & 36 & 0.60 & 15 & 5,990 & -- & -- & 0.50 & -- \\
\hline $44 \mathrm{~N} 31 \mathrm{~W} 16 \mathrm{CCC}$ & 210 & 0.40 & 13 & 1,880 & -- & -- & 0.20 & -- \\
\hline 44N32W14BABC & 15 & 0.40 & 43 & 431 & -- & -- & 11.0 & -- \\
\hline
\end{tabular}


Table 5. Physical properties and concentrations of inorganic and organic constituents from reconnaissance sampling of the Pierre aquifer-Continued

\begin{tabular}{|c|c|c|c|c|c|c|c|c|}
\hline Local number & $\begin{array}{c}\text { Arsenic, } \\
\text { dissolved } \\
(\mu g / L \\
\text { as As) } \\
(01000)\end{array}$ & $\begin{array}{c}\text { Barium, } \\
\text { dissolved } \\
(\mu \mathrm{g} / \mathrm{L} \\
\text { as Ba) } \\
(01005)\end{array}$ & $\begin{array}{c}\text { Cadmium, } \\
\text { dissolved } \\
(\mu g / L \\
\text { as Cd) } \\
(01025)\end{array}$ & $\begin{array}{c}\text { Chromium, } \\
\text { dissolved } \\
(\mu \mathrm{g} / \mathrm{L} \\
\text { as Cr) } \\
(01030)\end{array}$ & $\begin{array}{c}\text { Iron, } \\
\text { dissolved } \\
(\mu g / L \\
\text { as Fe) } \\
(01046)\end{array}$ & $\begin{array}{c}\text { Lead, } \\
\text { dissolved } \\
(\mu \mathrm{g} / \mathrm{L} \\
\text { as Pb) } \\
(01049)\end{array}$ & $\begin{array}{c}\text { Manganese, } \\
\text { dissolved } \\
(\mu g / L \\
\text { as Mn) } \\
(01056)\end{array}$ & $\begin{array}{c}\text { Mercury, } \\
\text { dissolved } \\
(\mu g / L \\
\text { as } \mathrm{Hg}) \\
(71890)\end{array}$ \\
\hline 40N25W 5ADC & - & -- & -- & -- & -- & -- & - & -- \\
\hline 41N27W21DDD & -- & -- & -- & -- & -- & -- & -- & -- \\
\hline 41N29W20DDD2 & -- & - & -- & -- & -- & -- & -- & -- \\
\hline 41N30W 7DDBD & -- & -- & -- & -- & -- & -- & -- & -- \\
\hline $42 \mathrm{~N} 26 \mathrm{~W} 14 \mathrm{AABA}$ & -- & -- & -- & -- & -- & -- & -- & -- \\
\hline $42 \mathrm{~N} 26 \mathrm{~W} 33 \mathrm{BDC}$ & -- & -- & -- & -- & -- & -- & -- & -- \\
\hline $42 \mathrm{~N} 27 \mathrm{~W} 9 \mathrm{BBB}$ & - & -- & -- & -- & -- & -- & -- & -- \\
\hline $42 \mathrm{~N} 30 \mathrm{~W} 13 \mathrm{ACDB}$ & $<1$ & $<100$ & $<1$ & $<1$ & 1,700 & $<1$ & 40 & $<0.1$ \\
\hline 42N30W31ADC2 & -- & -- & -- & -- & -- & -- & -- & -- \\
\hline $42 \mathrm{~N} 31 \mathrm{~W} 11 \mathrm{CCB}$ & -- & -- & -- & -- & -- & -- & -- & -- \\
\hline $42 \mathrm{~N} 32 \mathrm{~W} 9 \mathrm{CDB}$ & -- & -- & -- & -- & -- & -. & -- & -- \\
\hline 43N25W35AAA & -- & -- & -- & -- & -- & -- & -- & -- \\
\hline $43 \mathrm{~N} 27 \mathrm{~W} 36 \mathrm{CDDD}$ & -- & -- & -- & -- & -- & -- & - & -- \\
\hline $43 \mathrm{~N} 28 \mathrm{~W} 18 \mathrm{CCBC}$ & $<1$ & 18 & $<1$ & $<1$ & 10 & $<1$ & 100 & $<0.1$ \\
\hline 43N30W 4DAD & $<1$ & 100 & $<1$ & 2 & 80 & $<1$ & 500 & $<0.1$ \\
\hline 43N31W 8DBDC & $<1$ & $<100$ & $<1$ & 2 & 40 & $<1$ & 360 & $<0.1$ \\
\hline $44 \mathrm{~N} 30 \mathrm{~W} 28 \mathrm{DAC}$ & -- & -- & -- & -- & -- & -- & - & -- \\
\hline $44 N 31 \mathrm{~W} 16 \mathrm{CCC}$ & -- & -- & -- & -- & -- & -- & -- & -- \\
\hline $44 \mathrm{~N} 32 \mathrm{~W} 14 \mathrm{BABC}$ & 5 & 280 & 2 & $<1$ & 5 & $<1$ & $<1$ & $<0.1$ \\
\hline
\end{tabular}


Table 5. Physical properties and concentrations of inorganic and organic constituents from reconnaissance sampling of the Pierre aquifer-Continued

\begin{tabular}{|c|c|c|c|c|c|c|c|c|}
\hline Local number & $\begin{array}{c}\text { Selenium, } \\
\text { dissolved } \\
(\mu g / L \\
\text { as Se) } \\
(01145)\end{array}$ & $\begin{array}{c}\text { Zinc, } \\
\text { dissolved } \\
(\mu g / L \\
\text { as Zn) } \\
(01090)\end{array}$ & $\begin{array}{c}\text { Alachlor, } \\
\text { total, } \\
\text { recover } \\
(\mu g / L) \\
(77825)\end{array}$ & $\begin{array}{c}\text { Ametryne, } \\
\text { total } \\
(\mu \mathrm{g} / \mathrm{L}) \\
(82184)\end{array}$ & $\begin{array}{c}\text { Atrazine, } \\
\text { unfitrd, } \\
\text { recover } \\
(\mu g / L) \\
(39630)\end{array}$ & $\begin{array}{c}\text { Cyanazine, } \\
\text { total } \\
(\mu \mathrm{g} / \mathrm{L}) \\
(\mathbf{8 1 7 5 7 )}\end{array}$ & $\begin{array}{c}\text { Dicamba } \\
\text { (Mediben) } \\
\text { (Banvel D), } \\
\text { total } \\
(\mu \mathrm{g} / \mathrm{L}) \\
(82052)\end{array}$ & $\begin{array}{c}2,4,5-\mathrm{T}, \\
\text { total } \\
(\mu \mathrm{g} / \mathrm{L}) \\
(39740)\end{array}$ \\
\hline 40N25W 5ADC & -- & -- & -- & -- & - & -- & -- & - \\
\hline $41 \mathrm{~N} 27 \mathrm{~W} 21 \mathrm{DDD}$ & -- & -- & -- & -- & -- & -- & -- & -- \\
\hline 41N29W20DDD2 & -- & -- & -- & - & -- & -- & -- & -- \\
\hline 41N30W 7DDBD & - & -- & - & -- & -- & -- & -- & -- \\
\hline 42N26W14AABA & -- & -- & -- & -- & -- & -- & -- & -- \\
\hline $42 \mathrm{~N} 26 \mathrm{~W} 33 \mathrm{BDC}$ & -- & -- & -- & -- & -- & -- & -- & -- \\
\hline $42 \mathrm{~N} 27 \mathrm{~W} 9 \mathrm{BBB}$ & -- & -- & $<0.10$ & $<0.10$ & $<0.1$ & $<0.10$ & $<0.01$ & $<0.01$ \\
\hline 42N30W13ACDB & $<1$ & 10 & -- & -- & - & -- & -- & -- \\
\hline $42 \mathrm{~N} 30 \mathrm{~W} 31 \mathrm{ADC} 2$ & -- & -- & -- & -- & -- & -- & -- & -- \\
\hline $42 \mathrm{~N} 31 \mathrm{~W} 11 \mathrm{CCB}$ & -- & -- & -- & -- & -- & -- & -- & -- \\
\hline $42 \mathrm{~N} 32 \mathrm{~W} 9 \mathrm{CDB}$ & -- & -- & -- & -- & -- & -- & -- & -- \\
\hline 43N25W35AAA & -- & - & -- & -- & - & -- & -- & -- \\
\hline 43N27W36CDDD & -- & -- & -- & -- & -- & -- & - & -- \\
\hline $43 \mathrm{~N} 28 \mathrm{~W} 18 \mathrm{CCBC}$ & 4 & 55 & -- & -- & -- & -- & -- & - \\
\hline 43N30W 4DAD & 3 & 390 & -- & -- & -- & -- & -- & -- \\
\hline $43 N 31 W$ 8DBDC & 3 & 360 & -- & -- & -- & -- & -- & -- \\
\hline $44 \mathrm{~N} 30 \mathrm{~W} 28 \mathrm{DAC}$ & -- & -- & -- & - & -- & -- & -- & -- \\
\hline $44 \mathrm{~N} 31 \mathrm{~W} 16 \mathrm{CCC}$ & -- &.- & -- & -. & -- & -- & -- & -- \\
\hline 44N32W14BABC & 7 & 41 & -- & -- & -- & -- & -- & - \\
\hline
\end{tabular}


Table 5. Physical properties and concentrations of inorganic and organic constituents from reconnaissance sampling of the Pierre aquifer-Continued

\begin{tabular}{|c|c|c|c|c|c|c|c|c|c|}
\hline Local number & $\begin{array}{c}2,4-D \\
\text { total } \\
(\mu g / L) \\
(39730)\end{array}$ & $\begin{array}{c}\text { 2,4-DP, } \\
\text { total } \\
(\mu g / L) \\
(82183)\end{array}$ & $\begin{array}{c}\text { Picloram } \\
\text { (Tordon) } \\
\text { (Amdon), } \\
\text { total } \\
(\mu g / L) \\
(39720)\end{array}$ & $\begin{array}{c}\text { Prome- } \\
\text { tone, } \\
\text { total } \\
(\mu g / L) \\
(39056)\end{array}$ & $\begin{array}{c}\text { Prome- } \\
\text { tryne, } \\
\text { total } \\
(\mu g / L) \\
(39057)\end{array}$ & $\begin{array}{c}\text { Propazine, } \\
\text { total } \\
(\mu g / L) \\
(39024)\end{array}$ & $\begin{array}{c}\text { Silvex, } \\
\text { total } \\
(\mu g / L) \\
(39760)\end{array}$ & $\begin{array}{c}\text { Simazine, } \\
\text { total } \\
(\mu g / L) \\
(39055)\end{array}$ & $\begin{array}{c}\text { Simetryne, } \\
\text { total } \\
(\mu g / L) \\
(39054)\end{array}$ \\
\hline 40N25W 5ADC & -- & - & -- & -- & -- & -- & -- & - & -- \\
\hline $41 \mathrm{~N} 27 \mathrm{~W} 21 \mathrm{DDD}$ & -- & -- & -- & -- & -- & -- & -- & -- & -- \\
\hline 41N29W20DDD2 & -- & -- & -- & -- & -- & -- & -- & -- & -- \\
\hline 4IN30W 7DDBD & -- & -- & -- & -- & -- & -- & -- & -- & -- \\
\hline $42 \mathrm{~N} 26 \mathrm{~W} 14 \mathrm{AABA}$ & -- & -- & -- & -- & -- & -- & -- & -- & -- \\
\hline $42 \mathrm{~N} 26 \mathrm{~W} 33 \mathrm{BDC}$ & -- & -- & -- & -- & -- & - & -- & -- & -- \\
\hline $42 \mathrm{~N} 27 \mathrm{~W} 9 \mathrm{BBB}$ & $<0.01$ & $<0.01$ & $<0.01$ & $<0.10$ & $<0.10$ & $<0.10$ & $<0.01$ & $<0.10$ & $<0.10$ \\
\hline $42 \mathrm{~N} 30 \mathrm{~W} 13 \mathrm{ACDB}$ & -- & -- & -- & -- & -. & -- & -- & - & -- \\
\hline $42 \mathrm{~N} 30 \mathrm{~W} 31 \mathrm{ADC} 2$ & -- & -- & -- & -- & -- & -- & -- & -- &.- \\
\hline $42 \mathrm{~N} 31 \mathrm{~W} 11 \mathrm{CCB}$ & -- & -- & -- & -- & -- & -- & -- & -- & -- \\
\hline $42 \mathrm{~N} 32 \mathrm{~W} 9 \mathrm{CDB}$ & -- & -- & -- & -- & -- & -- & -- & -- & - \\
\hline $43 \mathrm{~N} 25 \mathrm{~W} 35 \mathrm{AAA}$ & - & -- & -- & -- & -- & -- & -- & -- & - \\
\hline 43N27W36CDDD & -- & -- & - & -- & -- & -- & -- & -- & -- \\
\hline $43 \mathrm{~N} 28 \mathrm{~W} 18 \mathrm{CCBC}$ & -- & -- & -- & -- & -- & -- & -- & -- & -- \\
\hline 43N30W 4DAD & -- & -- & -- & -- & -- & -- & $-\cdot$ & -- & -- \\
\hline $43 \mathrm{~N} 31 \mathrm{~W} 8 \mathrm{DBDC}$ & -- & -- & -- & -- & -- & -- & -- & -- & -- \\
\hline $44 \mathrm{~N} 30 \mathrm{~W} 28 \mathrm{DAC}$ & -- & -- & -- & -- & -- & -- & -- & -- & -- \\
\hline 44N31W16CCC & -- & -- & -- & -- & -- & -- & -- & -- & -- \\
\hline $44 \mathrm{~N} 32 \mathrm{~W} 14 \mathrm{BABC}$ & -- & -- & -- & -- & -- & -- & -- & -- & -- \\
\hline
\end{tabular}

${ }^{1}$ Laboratory value is given because field value was missing. 
Table 6. Physical properties and concentrations of inorganic constituents from reconnaissance sampling of the Dakota aquifer

[Agency collecting or analyzing sample: RST, Rosebud Sioux Tribe. $\mu \mathrm{S} / \mathrm{cm}$, microsiemens per centimeter; $\mathrm{mg} / \mathrm{L}, \mathrm{milligrams}$ per liter; $\mu \mathrm{g} / \mathrm{L}, \mathrm{micrograms}$ per liter; deg C, degrees Celsius; NTU, nephelometric turbidity units; $<$, less than; --, no data]

\begin{tabular}{|c|c|c|c|c|c|c|c|}
\hline Station number & Local number & County & Date & $\begin{array}{c}\text { Depth of } \\
\text { well } \\
\text { (feet) } \\
\text { (72008) }\end{array}$ & $\begin{array}{l}\text { Agency } \\
\text { collecting } \\
\text { sample }\end{array}$ & $\begin{array}{c}\text { Specific } \\
\text { conduct- } \\
\text { ance, } \\
\text { field } \\
(\mu \mathrm{S} / \mathrm{cm}) \\
(00095)\end{array}$ & $\begin{array}{c}\text { pH, } \\
\text { field } \\
\text { (standard } \\
\text { units) } \\
(\mathbf{0 0 4 0 0 )}\end{array}$ \\
\hline 433157100320501 & $41 \mathrm{~N} 27 \mathrm{~W} 16 \mathrm{BAA} 2$ & Mellette & $10-03-90$ & 2,140 & RST & 2,750 & 7.5 \\
\hline
\end{tabular}

\begin{tabular}{|c|c|c|c|c|c|c|c|c|c|}
\hline $\begin{array}{l}\text { Temper- } \\
\text { ature, } \\
\text { water } \\
\text { (deg C) } \\
\text { (00010) }\end{array}$ & $\begin{array}{c}\text { Turbidity } \\
\text { (NTU) } \\
\text { (00076) }\end{array}$ & $\begin{array}{c}\text { Alkalinity, } \\
\text { lab } \\
\left(\mathrm{mg}^{2} \text { as }\right. \\
\left.\mathrm{CaCO}_{3}\right) \\
(90410)\end{array}$ & $\begin{array}{c}\text { Calcium, } \\
\text { dissolved } \\
\text { (mg/L } \\
\text { as Ca) } \\
(00915)\end{array}$ & $\begin{array}{l}\text { Magne- } \\
\text { sium, } \\
\text { dissolved } \\
\text { (mg/L } \\
\text { as Mg) } \\
(00925)\end{array}$ & $\begin{array}{c}\text { Sodium, } \\
\text { dissolved } \\
\text { (mg/L } \\
\text { as } \mathrm{Na}) \\
(00930)\end{array}$ & $\begin{array}{l}\text { Sodium, } \\
\text { percent } \\
(00932)\end{array}$ & $\begin{array}{l}\text { Sodium } \\
\text { adsorption } \\
\text { ratio } \\
(00931)\end{array}$ & $\begin{array}{c}\text { Potassium, } \\
\text { dissolved } \\
\text { (mg/L } \\
\text { as K) } \\
(00935)\end{array}$ & $\begin{array}{c}\text { Sulfate, } \\
\text { dissolved } \\
\text { (mg/L } \\
\left.\text { as } \mathrm{SO}_{4}\right) \\
(00945)\end{array}$ \\
\hline 20.0 & 10 & 303 & 27 & 4.9 & 580 & 92 & 27 & 12 & 870 \\
\hline
\end{tabular}

\begin{tabular}{|c|c|c|c|c|c|c|c|c|}
\hline $\begin{array}{c}\text { Chloride, } \\
\text { dissolved } \\
\text { (mg/L } \\
\text { as Cl) } \\
(00940)\end{array}$ & $\begin{array}{c}\text { Fluoride, } \\
\text { dissolved } \\
\text { (mg/L } \\
\text { as F) } \\
(00950)\end{array}$ & $\begin{array}{c}\text { Silica, } \\
\text { dissolved } \\
(\mathrm{mg} / \mathrm{L} \\
\left.\text { as } \mathrm{Si}_{2}\right) \\
(00955)\end{array}$ & $\begin{array}{l}\text { Solids, } \\
\text { residue at } \\
180 \text { deg C, } \\
\text { dissolved } \\
(\mathrm{mg} / \mathrm{L}) \\
(70300)\end{array}$ & $\begin{array}{l}\text { Nitrogen, } \\
\text { ammonia, } \\
\text { dissolved } \\
\text { (mg/h } \\
\text { as } \mathrm{N}) \\
(00608)\end{array}$ & $\begin{array}{l}\text { Nitrogen, } \\
\text { nitrite, } \\
\text { dissolved } \\
\text { (mg/h } \\
\text { as } \mathrm{N}) \\
(00613)\end{array}$ & $\begin{array}{c}\text { Nitrogen, } \\
\mathrm{NO}_{2}+\mathrm{NO}_{3} \\
\text { dissolved } \\
\text { (mg/L } \\
\text { as } \mathrm{N} \text { ) } \\
(00631)\end{array}$ & $\begin{array}{l}\text { Phosphorus, } \\
\text { ortho, } \\
\text { dissolved } \\
\text { (mg/L } \\
\text { as P) } \\
(00671)\end{array}$ & $\begin{array}{c}\text { Arsenic, } \\
\text { dissolved } \\
(\mu \mathrm{g} / \mathrm{L} \\
\text { as As) } \\
(01000)\end{array}$ \\
\hline 48 & 2.2 & 27 & 1,890 & 1.60 & $<0.01$ & $<0.10$ & $<0.01$ & $<1$ \\
\hline
\end{tabular}

\begin{tabular}{|c|c|c|c|c|c|c|c|c|}
\hline $\begin{array}{c}\text { Barium, } \\
\text { dissolved } \\
(\mu \mathrm{g} / \mathrm{L} \\
\text { as Ba) } \\
(01005)\end{array}$ & $\begin{array}{c}\text { Cadmium, } \\
\text { dissolved } \\
(\mu \mathrm{g} / \mathrm{L} \\
\text { as Cd) } \\
(01025)\end{array}$ & $\begin{array}{c}\text { Chromium, } \\
\text { dissolved } \\
(\mu \mathrm{g} / \mathrm{L} \\
\text { as Cr) } \\
(01030)\end{array}$ & $\begin{array}{c}\text { Iron, } \\
\text { dissolved } \\
(\mu g / L \\
\text { as Fe) } \\
(01046)\end{array}$ & $\begin{array}{c}\text { Lead, } \\
\text { dissolved } \\
(\mu \mathrm{g} / \mathrm{L} \\
\text { as } \mathrm{Pb}) \\
(01049)\end{array}$ & $\begin{array}{c}\text { Manganese, } \\
\text { dissolved } \\
(\mu g / L \\
\text { as Mn) } \\
(01056)\end{array}$ & $\begin{array}{c}\text { Mercury, } \\
\text { dissolved } \\
(\mu \mathrm{g} / \mathrm{L} \\
\text { as } \mathrm{Hg}) \\
(71890)\end{array}$ & $\begin{array}{c}\text { Selenium, } \\
\text { dissolved } \\
(\mu \mathrm{g} / \mathrm{L} \\
\text { as Se) } \\
(01145)\end{array}$ & $\begin{array}{c}\text { Zinc, } \\
\text { dissolved } \\
(\mu \mathrm{g} / \mathrm{L} \\
\text { as } \mathrm{Zn}) \\
(01090)\end{array}$ \\
\hline$<100$ & $<1$ & $<1$ & 1,500 & $<1$ & 90 & $<0.1$ & $<1$ & 20 \\
\hline
\end{tabular}


Table 7. Physical properties and concentrations of inorganic constituents from reconnaissance sampling of unidentified aquifers

[Agency collecting or analyzing sample: RST, Rosebud Sioux Tribe; USGS, U.S. Geological Survey. $\mu$ S/cm, microsiemens per centimeter; mg/L, milligrams per liter; $\mu \mathrm{g} / \mathrm{L}$, micrograms per liter; deg C, degrees Celsius; NTU, nephelometric turbidity units; <, less than; --, no data]

\begin{tabular}{|c|c|c|c|c|c|c|c|c|}
\hline Station number & Local number & County & Date & $\begin{array}{c}\text { Depth of } \\
\text { well } \\
\text { (feet) } \\
(72008)\end{array}$ & $\begin{array}{l}\text { Agency } \\
\text { collecting } \\
\text { sample }\end{array}$ & $\begin{array}{c}\text { Specific } \\
\text { conduct- } \\
\text { ance } \\
(\mu S / \mathrm{cm}) \\
(00095)\end{array}$ & $\begin{array}{c}\mathrm{pH}, \\
\text { field } \\
\text { (standard } \\
\text { units) } \\
(00400)\end{array}$ & $\begin{array}{c}\text { Temper- } \\
\text { ature, } \\
\text { water } \\
\text { (deg C) } \\
(00010)\end{array}$ \\
\hline 433256100460001 & 41N29W10BAB & Mellette & $08-14-90$ & -- & RST & 608 & 7.0 & 17.0 \\
\hline 433438100204201 & $42 \mathrm{~N} 25 \mathrm{~W} 31 \mathrm{BBD}$ & Mellette & $08-16-90$ & -- & RST & 1,310 & 9.5 & 23.0 \\
\hline 430226100445501 & 35N29W 5ADDA & Todd & $07-19-90$ & -- & USGS & 342 & 7.4 & 13.0 \\
\hline 430415100493801 & 36N30W27ADAA & Todd & $08-01-90$ & -- & USGS & 1,190 & 7.0 & 15.5 \\
\hline 431253100535201 & 37N30W31DAB & Todd & $10-23-90$ & $\cdots$ & RST & 322 & 7.6 & 13.5 \\
\hline 430958101104701 & 37N33W23ADCA & Todd & $09-18-90$ & -- & RST & 306 & 7.6 & 20.0 \\
\hline
\end{tabular}

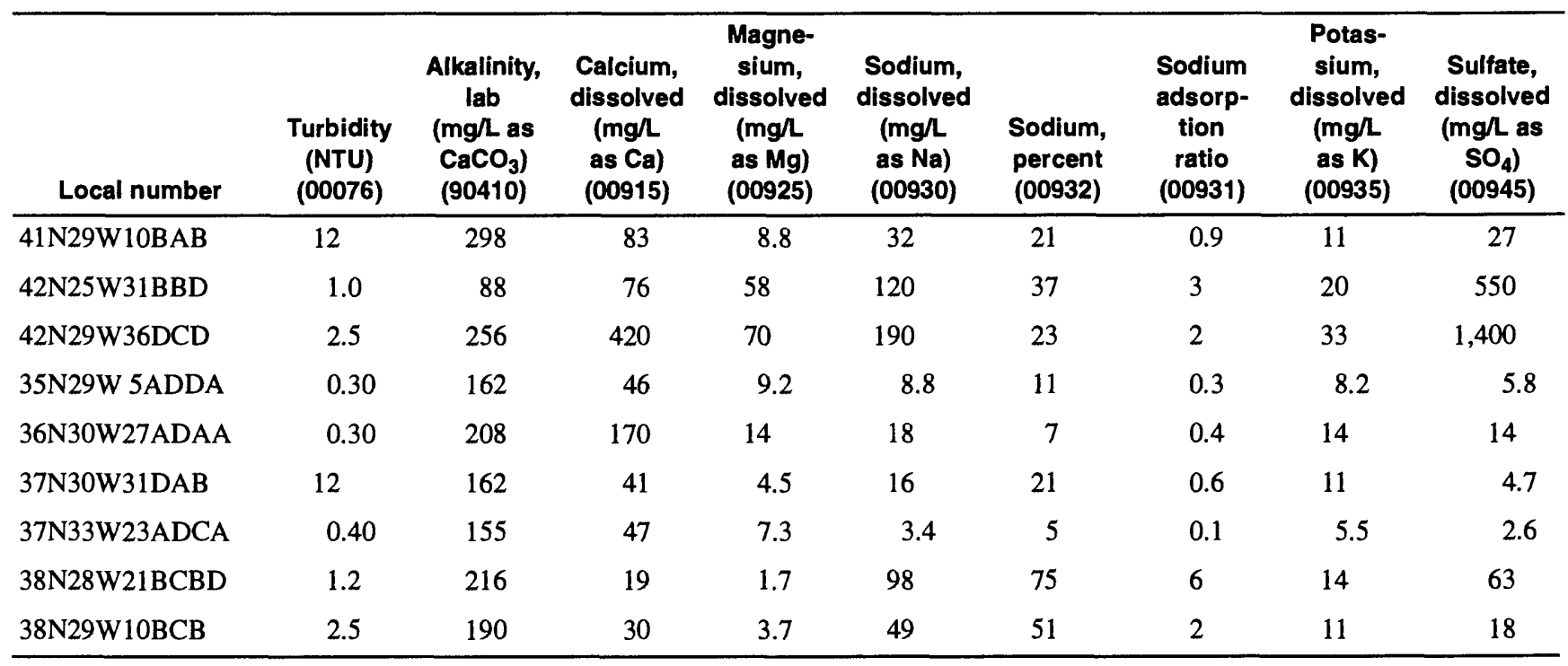


Table 7. Physical properties and concentrations of inorganic constituents from reconnaissance sampling of aquifers that could not be identified-Continued

\begin{tabular}{|c|c|c|c|c|c|c|c|c|}
\hline Local number & $\begin{array}{c}\text { Chloride, } \\
\text { dissolved } \\
\text { (mg/L } \\
\text { as Cl) } \\
(00940)\end{array}$ & $\begin{array}{c}\text { Fluoride, } \\
\text { dissolved } \\
\text { (mg/L } \\
\text { as F) } \\
(00950)\end{array}$ & $\begin{array}{c}\text { Silica, } \\
\text { dissolved } \\
\text { (mg/L } \\
\left.\text { as } \mathrm{SiO}_{2}\right) \\
(00955)\end{array}$ & $\begin{array}{c}\text { Solids, } \\
\text { residue } \\
\text { at } 180 \\
\text { deg C, } \\
\text { dissolved } \\
(\mathrm{mg} / \mathrm{L}) \\
(70300)\end{array}$ & $\begin{array}{c}\text { Nitrogen, } \\
\text { ammonia, } \\
\text { dissolved } \\
\text { (mg/L } \\
\text { as N) } \\
(00608)\end{array}$ & $\begin{array}{c}\text { Nitrogen, } \\
\text { nitrite, } \\
\text { dissolved } \\
\text { (mg/h } \\
\text { as N) } \\
(00613)\end{array}$ & $\begin{array}{c}\text { Nitrogen, } \\
\mathrm{NO}_{2}+\mathrm{NO}_{3} \\
\text { dissolved } \\
(\mathrm{mg} / \mathrm{L} \\
\text { as } \mathrm{N}) \\
(00631)\end{array}$ & $\begin{array}{c}\text { Phos- } \\
\text { phorus, } \\
\text { ortho, } \\
\text { dissolved } \\
\text { (mg/h } \\
\text { as P) } \\
(00671)\end{array}$ \\
\hline $41 \mathrm{~N} 29 \mathrm{~W} 10 \mathrm{BAB}$ & 4.8 & 0.40 & 39 & 332 & - & $\overline{--}$ & $<0.10$ & - \\
\hline $42 \mathrm{~N} 25 \mathrm{~W} 31 \mathrm{BBD}$ & 7.6 & 0.30 & 0.60 & 964 & -- & -- & $<0.10$ & -- \\
\hline $42 \mathrm{~N} 29 \mathrm{~W} 36 \mathrm{DCD}$ & 26 & 0.30 & 13 & 2,400 & -- & -- & 0.80 & -- \\
\hline 35N29W 5ADDA & 5.5 & 0.30 & 77 & 239 & - & -- & 1.30 & - \\
\hline 36N30W27ADAA & 37 & 0.30 & 59 & 992 & -- & -- & 77.0 & -- \\
\hline 37N30W31DAB & 3.2 & 0.20 & 70 & 235 & 0.02 & $<0.01$ & 0.60 & 0.01 \\
\hline 37N33W23ADCA & 3.3 & 0.30 & 50 & 188 & .. & -- & 1.30 & -- \\
\hline $38 \mathrm{~N} 28 \mathrm{~W} 21 \mathrm{BCBD}$ & 4.3 & 0.60 & 62 & 386 & - & -- & 1.30 & -- \\
\hline $38 \mathrm{~N} 29 \mathrm{~W} 10 \mathrm{BCB}$ & 4.3 & 0.50 & 72 & 291 & -- & -- & 0.90 & -. \\
\hline
\end{tabular}


Table 8. Quality-assurance results of field blank sample collected during the reconnaissance sampling program $[\mu \mathrm{S} / \mathrm{cm}$, microsiemens per centimeter; mg/L, milligrams per liter; deg C, degrees Celsius; NTU, nephelometric turbidity units; <, less than; --, no data]

\begin{tabular}{|c|c|c|c|c|c|c|c|c|}
\hline Station name & Local number & Date & $\begin{array}{c}\text { Specific } \\
\text { conduct- } \\
\text { ance, } \\
\text { lab } \\
(\mu S / \mathrm{cm}) \\
(00095)\end{array}$ & $\begin{array}{c}\text { pH, } \\
\text { lab } \\
\text { (standard } \\
\text { units) } \\
(00400)\end{array}$ & $\begin{array}{c}\text { Turbidity } \\
\text { (NTU) } \\
\text { (00076) }\end{array}$ & $\begin{array}{c}\text { Alkalinity, } \\
\text { lab } \\
(\mathrm{mg} / \mathrm{L} \text { as } \\
\left.\mathrm{CaCO}_{3}\right) \\
(90410)\end{array}$ & $\begin{array}{c}\text { Calcium, } \\
\text { dissolved } \\
\text { (mg/L } \\
\text { as Ca) } \\
\text { (00915) }\end{array}$ & $\begin{array}{l}\text { Magne- } \\
\text { sium, } \\
\text { dissolved } \\
\text { (mg/l } \\
\text { as } \mathrm{Mg}) \\
(00925)\end{array}$ \\
\hline 431253100535201 & 37N30W31DAB & $10-23-90$ & 6 & 6.6 & 0.40 & 4.4 & 0.20 & 0.06 \\
\hline
\end{tabular}

\begin{tabular}{|c|c|c|c|c|c|c|c|c|c|c|}
\hline $\begin{array}{c}\text { Sodium, } \\
\text { dissolved } \\
\text { (mg/L } \\
\text { as } \mathrm{Na}) \\
(00930)\end{array}$ & $\begin{array}{l}\text { Potas- } \\
\text { sium, } \\
\text { dissolved } \\
\text { (mg/L } \\
\text { as K) } \\
(00935)\end{array}$ & $\begin{array}{c}\text { Sulfate, } \\
\text { dissolved } \\
\text { (mg/l as } \\
\left.\mathrm{SO}_{4}\right) \\
(00945)\end{array}$ & $\begin{array}{c}\text { Chloride, } \\
\text { dissolved } \\
\text { (mg/L } \\
\text { as Cl) } \\
(00940)\end{array}$ & $\begin{array}{c}\text { Fluoride, } \\
\text { dissolved } \\
\text { (mg/L } \\
\text { as F) } \\
(00950)\end{array}$ & $\begin{array}{c}\text { Silica, } \\
\text { dissolved } \\
(\mathbf{m g} / \mathrm{L} \\
\left.\text { as } \mathrm{SiO}_{2}\right) \\
(00955)\end{array}$ & $\begin{array}{c}\text { Solids, } \\
\text { residue } \\
\text { at } 180 \\
\text { deg C, } \\
\text { dissolved } \\
\text { (mg/L) } \\
(70300)\end{array}$ & $\begin{array}{l}\text { Nitrogen, } \\
\text { ammonia, } \\
\text { dissolved } \\
\text { (mg/L } \\
\text { as } N \text { ) } \\
(00608)\end{array}$ & $\begin{array}{l}\text { Nitrogen, } \\
\text { nitrite, } \\
\text { dissolved } \\
\text { (mg/L } \\
\text { as N) } \\
(00613)\end{array}$ & $\begin{array}{c}\text { Nitrogen, } \\
\mathrm{NO}_{2}+\mathrm{NO}_{3} \\
\text { dissolved } \\
\text { (mg/L } \\
\text { as } \mathrm{N} \text { ) } \\
(00631)\end{array}$ & $\begin{array}{c}\text { Phos- } \\
\text { phorus, } \\
\text { ortho, } \\
\text { dissolved } \\
\text { (mg/L } \\
\text { as P) } \\
\text { (00671) }\end{array}$ \\
\hline 1.1 & 0.10 & 1.0 & 0.40 & 0.10 & 1.1 & 2 & 0.02 & $<0.01$ & $<0.10$ & 0.02 \\
\hline
\end{tabular}


Table 9. Chemical analyses of field duplicates for reconnaissance water samples

$[\mu \mathrm{S} / \mathrm{cm}$, microsiemens per centimeter; mg/L, milligrams per liter; $\mu \mathrm{g} / \mathrm{L}$, micrograms per liter; deg C, degrees Celsius; NTU, nephelometric turbidity units; $<$, less than; --, no data]

\begin{tabular}{|c|c|c|c|c|c|c|c|c|}
\hline Station name & Local number & $\begin{array}{c}\text { Sample } \\
\text { type }\end{array}$ & Aquifer & Date & $\begin{array}{c}\text { Specific } \\
\text { conduct- } \\
\text { ance, } \\
(\mu \mathrm{S} / \mathrm{cm}) \\
(00095)\end{array}$ & $\begin{array}{c}\text { pH, } \\
\text { (standard } \\
\text { units) } \\
(00400)\end{array}$ & $\begin{array}{c}\text { Turbidity } \\
\text { (NTU) } \\
\text { (00076) }\end{array}$ & $\begin{array}{c}\text { Alkalinity, } \\
\text { (mg/L as } \\
\left.\mathrm{CaCO}_{3}\right) \\
(90410)\end{array}$ \\
\hline 430504100471201 & 36N29W19BCBA & Sample & Ogallala & $10-15-90$ & 328 & 7.6 & 0.50 & 161 \\
\hline 430504100471201 & $36 \mathrm{~N} 29 \mathrm{~W} 19 \mathrm{BCBA}$ & Duplicate & -- & $10-15-90$ & 325 & 7.8 & 0.40 & 161 \\
\hline 432558100513303 & $40 \mathrm{~N} 30 \mathrm{~W} 22 \mathrm{BAB}$ & Sample & White $\mathrm{R}$ & $10-16-90$ & 480 & 7.3 & 0.40 & 213 \\
\hline 432558100513303 & $40 \mathrm{~N} 30 \mathrm{~W} 22 \mathrm{BAB}$ & Duplicate & -- & $10-16-90$ & 464 & 7.6 & 0.60 & 213 \\
\hline 432728100562703 & $40 \mathrm{~N} 31 \mathrm{~W} 12 \mathrm{BDB} 3$ & Sample & White $\mathrm{R}$ & $10-16-90$ & 910 & 7.1 & 0.40 & 422 \\
\hline 432728100562703 & $40 \mathrm{~N} 31 \mathrm{~W} 12 \mathrm{BDB} 3$ & Duplicate & -- & $10-16-90$ & 906 & 7.5 & 0.60 & 423 \\
\hline 432832101105201 & $40 \mathrm{~N} 33 \mathrm{~W} 2 \mathrm{AAB}$ & Sample & White R & $10-02-90$ & 567 & 7.1 & 0.40 & 288 \\
\hline 432832101105201 & $40 \mathrm{~N} 33 \mathrm{~W} 2 \mathrm{AAB}$ & Duplicate & -- & $10-02-90$ & 572 & 7.5 & 0.70 & 288 \\
\hline 433033100315201 & $41 \mathrm{~N} 27 \mathrm{~W} 21 \mathrm{DDD}$ & Sample & Pierre & $10-22-90$ & 2,930 & 6.7 & 0.50 & 526 \\
\hline 433033100315201 & $41 N 27 W 21 D D D$ & Duplicate & -- & $10-22-90$ & 2,880 & 7.0 & 0.50 & 368 \\
\hline
\end{tabular}

\begin{tabular}{|c|c|c|c|c|c|c|c|}
\hline Local number & $\begin{array}{c}\text { Calcium, } \\
\text { dissolved } \\
\text { (mg/L as Ca) } \\
(00915)\end{array}$ & $\begin{array}{c}\text { Magnesium, } \\
\text { dissolved } \\
\text { (mg/L as Mg) } \\
(00925)\end{array}$ & $\begin{array}{c}\text { Sodium, } \\
\text { dissolved } \\
\text { (mg/L as } \mathrm{Na}) \\
(00930)\end{array}$ & $\begin{array}{c}\text { Potassium, } \\
\text { dissolved } \\
\text { (mg/L as K) } \\
(00935)\end{array}$ & $\begin{array}{c}\text { Sulfate, } \\
\text { dissolved } \\
\left(\mathrm{mg} / \mathrm{L} \text { as } \mathrm{SO}_{4}\right) \\
(00945)\end{array}$ & $\begin{array}{c}\text { Chloride, } \\
\text { dissolved } \\
\text { (mg/L as Cl) } \\
(00940)\end{array}$ & $\begin{array}{c}\text { Fluoride, } \\
\text { dissolved } \\
\text { (mg/L as F) } \\
(00950)\end{array}$ \\
\hline $36 \mathrm{~N} 29 \mathrm{~W} 19 \mathrm{BCBA}$ & 48 & 8.9 & 3.6 & 7.4 & 2.0 & 3.3 & 0.30 \\
\hline $36 \mathrm{~N} 29 \mathrm{~W} 19 \mathrm{BCBA}$ & 48 & 8.9 & 3.5 & 7.5 & 1.9 & 3.2 & 0.30 \\
\hline $40 \mathrm{~N} 30 \mathrm{~W} 22 \mathrm{BAB}$ & 58 & 6.1 & 29 & 11 & 16 & 6.1 & 0.30 \\
\hline $40 \mathrm{~N} 30 \mathrm{~W} 22 \mathrm{BAB}$ & 58 & 6.1 & 29 & 11 & 15 & 6.2 & 0.20 \\
\hline 40N31W12BDB3 & 44 & 2.5 & 160 & 11 & 37 & 21 & 0.20 \\
\hline $40 \mathrm{~N} 31 \mathrm{~W} 12 \mathrm{BDB} 3$ & 45 & 2.7 & 160 & 11 & 37 & 22 & 0.20 \\
\hline $40 \mathrm{~N} 33 \mathrm{~W} 2 \mathrm{AAB}$ & 66 & 3.0 & 58 & 9.0 & 18 & 5.3 & $<0.10$ \\
\hline $40 \mathrm{~N} 33 \mathrm{~W} 2 \mathrm{AAB}$ & 64 & 2.9 & 56 & 8.9 & 17 & 5.6 & $<0.10$ \\
\hline $41 \mathrm{~N} 27 \mathrm{~W} 21 \mathrm{DDD}$ & 490 & 54 & 140 & 24 & 1,200 & 83 & 0.30 \\
\hline $41 \mathrm{~N} 27 \mathrm{~W} 21 \mathrm{DDD}$ & 500 & 55 & 140 & 24 & 1,200 & 83 & 0.30 \\
\hline
\end{tabular}


Table 9. Chemical analyses of field duplicates for reconnaissance water samples-Continued

\begin{tabular}{|c|c|c|c|c|c|c|c|}
\hline Local number & $\begin{array}{c}\text { Silica, } \\
\text { dissolved } \\
\text { (mg/L } \\
\text { as Si0 }) \\
(00955)\end{array}$ & $\begin{array}{c}\text { Solids, } \\
\text { residue at } \\
180 \text { deg C, } \\
\text { dissolved } \\
\text { (mg/L) } \\
(70300)\end{array}$ & $\begin{array}{c}\text { Nitrogen, } \\
\text { ammonia, } \\
\text { dissolved } \\
\text { (mg/L as N) } \\
\text { (00608) }\end{array}$ & $\begin{array}{c}\text { Nitrogen, } \\
\text { nitrite, } \\
\text { dissolved } \\
\text { (mg/L as N) } \\
\text { (00613) }\end{array}$ & $\begin{array}{c}\text { Nitrogen, } \\
\mathrm{NO}_{2}+\mathrm{NO}_{3} \\
\text { dissolved } \\
\text { (mg/L as N) } \\
\text { (00631) }\end{array}$ & $\begin{array}{l}\text { Phosphorus, } \\
\text { ortho, } \\
\text { dissolved } \\
\text { (mg/L as P) } \\
\text { (00671) }\end{array}$ & $\begin{array}{c}\text { Arsenic, } \\
\text { dissolved } \\
(\mu g / L \\
\text { as As) } \\
(01000)\end{array}$ \\
\hline 36N29W19BCBA & 57 & 230 & $<0.01$ & $<0.01$ & 2.20 & 0.02 & -- \\
\hline 36N29W19BCBA & 57 & 229 & $<0.01$ & $<0.01$ & 2.20 & 0.02 & -- \\
\hline $40 \mathrm{~N} 30 \mathrm{~W} 22 \mathrm{BAB}$ & 48 & 299 & 0.01 & $<0.01$ & 4.40 & 0.14 & 10 \\
\hline $40 \mathrm{~N} 30 \mathrm{~W} 22 \mathrm{BAB}$ & 48 & 304 & 0.01 & $<0.01$ & 4.50 & 0.18 & 10 \\
\hline 40N31W12BDB3 & 47 & 575 & 0.02 & $<0.01$ & 2.30 & 0.03 & -- \\
\hline 40N31W12BDB3 & 47 & 595 & 0.01 & $<0.01$ & 2.20 & 0.03 & - \\
\hline $40 \mathrm{~N} 33 \mathrm{~W} 2 \mathrm{AAB}$ & 65 & 383 & $<0.01$ & $<0.01$ & $<0.10$ & 0.02 & -- \\
\hline $40 \mathrm{~N} 33 \mathrm{~W} 2 \mathrm{AAB}$ & 65 & 391 & $<0.01$ & $<0.01$ & $<0.10$ & 0.01 & -- \\
\hline $41 \mathrm{~N} 27 \mathrm{~W} 21 \mathrm{DDD}$ & 34 & 2,600 & 0.04 & $<0.01$ & 22.0 & 0.02 & -- \\
\hline $41 \mathrm{~N} 27 \mathrm{~W} 21 \mathrm{DDD}$ & 34 & 2,440 & 0.04 & $<0.01$ & 22.0 & 0.03 & - \\
\hline
\end{tabular}

\begin{tabular}{|c|c|c|c|c|c|c|c|c|c|}
\hline Local number & $\begin{array}{c}\text { Barium, } \\
\text { dissolved } \\
(\mu g / 2 \\
\text { as Ba) } \\
(01005)\end{array}$ & $\begin{array}{c}\text { Cadmium, } \\
\text { dissolved } \\
(\mu g / L \\
\text { as Cd) } \\
(01025)\end{array}$ & $\begin{array}{c}\text { Chromium, } \\
\text { dissolved } \\
(\mu g / L \\
\text { as Cr) } \\
(01030)\end{array}$ & $\begin{array}{c}\text { Iron, } \\
\text { dissolved } \\
(\mu g / L \\
\text { as Fe) } \\
(01046)\end{array}$ & $\begin{array}{c}\text { Lead, } \\
\text { dissolved } \\
(\mu g / \mathrm{h} \\
\text { as Pb) } \\
(01049)\end{array}$ & $\begin{array}{c}\text { Manga- } \\
\text { nese, } \\
\text { dissolved } \\
(\mu g / L \\
\text { as } M n) \\
(01056)\end{array}$ & $\begin{array}{c}\text { Mercury, } \\
\text { dissolved } \\
(\mu \mathrm{g} / \mathrm{L} \\
\text { as } \mathrm{Hg}) \\
(71890)\end{array}$ & $\begin{array}{c}\text { Selenium, } \\
\text { dissolved } \\
(\mu g / L \\
\text { as Se) } \\
(01145)\end{array}$ & $\begin{array}{c}\text { Zinc, } \\
\text { dissolved } \\
(\mu g / L \\
\text { as } \mathrm{Zn}) \\
(01090)\end{array}$ \\
\hline $36 \mathrm{~N} 29 \mathrm{~W} 19 \mathrm{BCBA}$ & -- & - & -- & -- & -- & -- & -- & -- & -- \\
\hline $36 \mathrm{~N} 29 \mathrm{~W} 19 \mathrm{BCBA}$ & - & -- & - & -- & -- & -- & -- & -- & -- \\
\hline $40 \mathrm{~N} 30 \mathrm{~W} 22 \mathrm{BAB}$ & 270 & $<1$ & 2 & 3 & $<1$ & $<1$ & $<0.1$ & 4 & 11 \\
\hline $40 \mathrm{~N} 30 \mathrm{~W} 22 \mathrm{BAB}$ & 280 & $<1$ & 2 & $<3$ & $<1$ & $<1$ & $<0.1$ & 4 & 10 \\
\hline $40 \mathrm{~N} 31 \mathrm{~W} 12 \mathrm{BDB} 3$ & -- & -- & -- & -- & -- & -- & -- & -- & -- \\
\hline $40 \mathrm{~N} 31 \mathrm{~W} 12 \mathrm{BDB} 3$ & -- & -- & -- & -- & -- & -- & -- & -- & $-\cdot$ \\
\hline $40 \mathrm{~N} 33 \mathrm{~W} 2 \mathrm{AAB}$ & -- & -- & -- & -- & -- & -- & -- & -- & -- \\
\hline $40 \mathrm{~N} 33 \mathrm{~W} 2 \mathrm{AAB}$ & -- & -- & -- & -- & -- & -- & -- & -- & -- \\
\hline $41 \mathrm{~N} 27 \mathrm{~W} 21 \mathrm{DDD}$ & -- & -- & -- & -- & -- & -- & -- & -- & -- \\
\hline 41N27W21DDD & -- & -- & -- & -- & -- & -- & -- & -- & -- \\
\hline
\end{tabular}


Table 10. Precision of chemical analyses of field duplicates for reconnaissance water samples

$[\mu \mathrm{S} / \mathrm{cm}$, microsiemens per centimeter; NTU, nephelometric turbidity units; $\mathrm{mg} / \mathrm{L}$, milligrams per liter; $\mu \mathrm{g} / \mathrm{L}$, micrograms per liter]

\begin{tabular}{|c|c|c|c|}
\hline Constituent and reporting unit & $\begin{array}{c}\text { Number of } \\
\text { duplicate pairs }\end{array}$ & $\begin{array}{c}\text { Standard deviation, } \\
\text { in units }\end{array}$ & $\begin{array}{l}\text { Relative standard deviation, } \\
\text { in percent }\end{array}$ \\
\hline Specific conductance, $\mu \mathrm{S} / \mathrm{cm}$ & 5 & 16.8 & 1.6 \\
\hline Turbidity, NTU & 5 & 0.13 & 27 \\
\hline Alkalinity, $\mathrm{mg} / \mathrm{L}$ as calcium carbonate & 5 & 50 & 16 \\
\hline Calcium, mg/L & 5 & 3.2 & 2.3 \\
\hline Magnesium, mg/L & 5 & 0.32 & 2.2 \\
\hline Sodium, mg/L & 5 & 0.63 & 0.81 \\
\hline Potassium, mg/L & 5 & 0.04 & 0.36 \\
\hline Sulfate, $\mathrm{mg} / \mathrm{L}$ & 5 & 0.45 & 0.18 \\
\hline Chloride, mg/L & 5 & 0.33 & 1.4 \\
\hline Fluoride, mg/L & 5 & 0.03 & 14 \\
\hline Silica, mg/L & 5 & 0.0 & 0.0 \\
\hline Solids, mg/L & 5 & 51 & 6.4 \\
\hline Ammonia, $\mathrm{mg} / \mathrm{L}$ as $\mathrm{N}$ & 5 & 0.003 & 21 \\
\hline Nitrite, $\mathrm{mg} / \mathrm{L}$ as $\mathrm{N}$ & 5 & 0.0 & 0.0 \\
\hline Nitrite + Nitrate, $\mathrm{mg} / \mathrm{L}$ as $\mathrm{N}$ & 5 & 0.04 & 0.72 \\
\hline Phosphorus, ortho, $\mathrm{mg} / \mathrm{L}$ as $\mathrm{P}$ & 5 & 0.01 & 26 \\
\hline Arsenic, $\mu \mathrm{g} / \mathrm{L}$ & 1 & 0.0 & 0.0 \\
\hline Barium, $\mu \mathrm{g} / \mathrm{L}$ & 1 & 7.1 & 2.6 \\
\hline Cadmium, $\mu \mathrm{g} / \mathrm{L}$ & 1 & 0.0 & 0.0 \\
\hline Chromium, $\mu \mathrm{g} / \mathrm{L}$ & 1 & 0.0 & 0.0 \\
\hline Iron, $\mu \mathrm{g} / \mathrm{L}$ & 1 & 1.1 & 47 \\
\hline Lead, $\mu \mathrm{g} / \mathrm{L}$ & 1 & 0.0 & 0.0 \\
\hline Manganese, $\mu \mathrm{g} / \mathrm{L}$ & 1 & 0.0 & 0.0 \\
\hline Mercury, $\mu \mathrm{g} / \mathrm{L}$ & 1 & 0.0 & 0.0 \\
\hline Selenium, $\mu \mathrm{g} / \mathrm{L}$ & 1 & 0.0 & 0.0 \\
\hline Zinc, $\mu \mathrm{g} / \mathrm{L}$ & 1 & 0.71 & 6.7 \\
\hline
\end{tabular}


Table 11. Selected water-quality data from observation wells and private wells completed in the Ogallala aquifer sampled by the U.S. Geological Survey for the nitrate studies

$[\mu \mathrm{S} / \mathrm{cm}$, microsiemens per centimeter; $\mathrm{mg} / \mathrm{L}$, milligrams per liter; $\mathrm{mm}$, millimeter; $\mu \mathrm{g} / \mathrm{L}$, micrograms per liter; deg $\mathrm{C}$, degrees Celsius; FET, fixed end point titration; IT, incremental titration; NTU, nephelometric turbidity units; recover, recoverable; unfltrd, unfiltered; <, less than; --, no data]

\begin{tabular}{|c|c|c|c|c|c|c|c|}
\hline Station number & Local number & Other identifier & Date & $\begin{array}{c}\text { Depth of } \\
\text { well } \\
\text { (feet) } \\
(72008)\end{array}$ & $\begin{array}{c}\text { Water level } \\
\text { (feet) } \\
\text { (72019) } \\
\end{array}$ & $\begin{array}{c}\text { Speciflc } \\
\text { conduct- } \\
\text { ance, } \\
\text { field } \\
(\mu \mathrm{S} / \mathrm{cm}) \\
(00095)\end{array}$ & 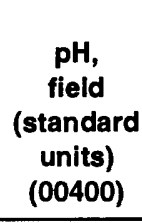 \\
\hline 430226100445203 & $35 \mathrm{~N} 29 \mathrm{~W}$ 4BCCB3 & $\mathrm{R} 20-90-36$ & $08-12-91$ & 27.0 & -- & 822 & 7.1 \\
\hline \multirow[t]{3}{*}{430652100450601} & $36 \mathrm{~N} 29 \mathrm{~W} 8 \mathrm{~A}$ & Gunnink domestic & 09-19-90 & 190.0 & -- & ${ }^{1} 417$ & ${ }^{1} 7.6$ \\
\hline & & & $08-06-91$ & 190.0 & -- & 424 & 7.3 \\
\hline & & & $09-14-94$ & 190.0 & -- & 397 & 7.4 \\
\hline 430705100450201 & $36 \mathrm{~N} 29 \mathrm{~W} 8 \mathrm{AABA}$ & R20-94-48 & $09-14-94$ & 77.0 & 66.05 & 411 & 7.4 \\
\hline 430613100445901 & 36N29W17AAAB & R20-94-47 & $09-13-94$ & 69.0 & 57.56 & 270 & 7.4 \\
\hline 430604100445403 & 36N29W17AADD3 & R20-90-32 & 09-13-94 & 78.0 & 69.86 & 383 & 7.3 \\
\hline 430600100450801 & 36N29W17ACAA & R20-90-33 & $09-13-94$ & 107.0 & 95.58 & 460 & 7.3 \\
\hline 430556100452501 & $36 \mathrm{~N} 29 \mathrm{~W} 17 \mathrm{ACBC} 2$ & R20-91-25 & $09-14-94$ & 108.0 & 92.62 & 445 & 7.4 \\
\hline \multirow[t]{3}{*}{430600100450901} & 36N29W17ADBB & Vanderwey domestic & $07-19-90$ & -- & -- & 743 & 7.2 \\
\hline & & & $08-06-91$ & -- & -- & 616 & 7.3 \\
\hline & & & $09-13-94$ & -- & -- & 590 & 7.4 \\
\hline 430603100460501 & 36N29W18AADD & R20-91-26 & $09-12-94$ & 123.0 & 98.94 & 340 & 7.4 \\
\hline \multirow[t]{2}{*}{430415100451401} & 36N29W29ACAA & Tribal well \#7 & $07-18-90$ & 133.5 & - & 385 & 7.4 \\
\hline & & & $08-06-91$ & 133.5 & -- & 382 & 7.5 \\
\hline 430858100445503 & $37 \mathrm{~N} 29 \mathrm{~W} 27 \mathrm{CBBC} 3$ & R20-90-04 & $08-12-91$ & 78.0 & -- & 444 & 7.5 \\
\hline \multirow[t]{3}{*}{430823100441201} & 37N29W34ACA & Ladely domestic & $07-19-90$ & 150.0 & -- & 710 & 6.9 \\
\hline & & & $08-06-91$ & 150.0 & -- & 688 & 6.8 \\
\hline & & & $09-12-94$ & 150.0 & -- & 598 & 7.1 \\
\hline
\end{tabular}


Table 11. Selected water-quality data from observation wells and private wells completed in the Ogallala aquifer sampled by the U.S. Geological Survey for the nitrate studies-Continued

\begin{tabular}{|c|c|c|c|c|c|c|c|c|c|}
\hline Local number & Date & $\begin{array}{l}\text { Temper- } \\
\text { ature, } \\
\text { air, } \\
\text { field } \\
\text { (deg C) } \\
(00020)\end{array}$ & $\begin{array}{l}\text { Temper- } \\
\text { ature, } \\
\text { water } \\
\text { (deg C) } \\
(00010)\end{array}$ & $\begin{array}{c}\text { Turbidity, } \\
\text { field } \\
\text { (NTU) } \\
\text { (00076) }\end{array}$ & $\begin{array}{c}\text { Baro- } \\
\text { metric } \\
\text { pressure, } \\
\text { field } \\
(\mathrm{mm} \\
\text { of } \mathrm{Hg}) \\
(00025)\end{array}$ & $\begin{array}{c}\text { Dis- } \\
\text { solved } \\
\text { oxygen, } \\
\text { field } \\
(\mathrm{mg} / \mathrm{L}) \\
(00300)\end{array}$ & $\begin{array}{l}\text { Dissolved } \\
\text { oxygen, } \\
\text { percent } \\
\text { saturation, } \\
\text { field } \\
\text { (00301) }\end{array}$ & $\begin{array}{c}\text { Alkalinity, } \\
\text { field, } \\
\text { dissolved, } \\
\text { IT } \\
\left(\mathrm{mgg}^{2} \text { as }\right. \\
\left.\mathrm{CaCO}_{3}\right) \\
(39086)\end{array}$ & $\begin{array}{c}\text { Alkalinity, } \\
\text { dissolved, } \\
\text { FET, } \\
\text { field } \\
\left(\mathrm{mg}^{2} \mathrm{~L} \text { as }\right. \\
\left.\mathrm{CaCO}_{3}\right) \\
(00418)\end{array}$ \\
\hline $35 \mathrm{~N} 29 \mathrm{~W} 4 \mathrm{BCCB} 3$ & $08-12-91$ & 23.5 & 13.0 & -- & -- & -- & -- & -- & -- \\
\hline \multirow[t]{3}{*}{$36 \mathrm{~N} 29 \mathrm{~W} 8 \mathrm{~A}$} & $09-19-90$ & -- & -- & 0.40 & -- & -- & -- & -- & -- \\
\hline & $08-06-91$ & 33.0 & 15.5 & -- & -- & -- & -- & -- & -- \\
\hline & $09-14-94$ & 31.5 & 13.5 & -. & 677 & 9.7 & 105 & 167 & 168 \\
\hline 36N29W 8AABA & $09-14-94$ & 31.5 & 14.5 & -- & 674 & 6.5 & 72 & 156 & 156 \\
\hline 36N29W17AAAB & $09-13-94$ & 17.5 & 12.0 & -- & 679 & 10.3 & 108 & 147 & 148 \\
\hline 36N29W17AADD3 & $09-13-94$ & 21.5 & 12.5 & -- & 681 & 9.8 & 103 & 220 & 213 \\
\hline 36N29W17ACAA & $09-13-94$ & 27.5 & 13.0 & -- & 679 & 10.3 & 110 & 194 & 190 \\
\hline $36 \mathrm{~N} 29 \mathrm{~W} 17 \mathrm{ACBC} 2$ & $09-14-94$ & 29.5 & 13.0 & -- & 676 & 10.5 & 112 & 162 & 162 \\
\hline \multirow[t]{3}{*}{ 36N29W17ADBB } & $07-19-90$ & 21.0 & 13.5 & 0.30 & -- & -- & -- & - & - \\
\hline & $08-06-91$ & 32.0 & 15.5 & -- & -- & -- & -- & -- & -- \\
\hline & $09-13-94$ & 27.5 & 13.5 & -- & 678 & 9.5 & 102 & 173 & 173 \\
\hline 36N29W18AADD & $09-12-94$ & 29.0 & 13.5 & -- & 676 & 11.6 & 126 & 149 & 150 \\
\hline \multirow[t]{2}{*}{$36 \mathrm{~N} 29 \mathrm{~W} 29 \mathrm{ACAA}$} & $07-18-90$ & 29.0 & 13.5 & 0.40 & -- & -- & -- & -- & -- \\
\hline & $08-06-91$ & 31.0 & 13.5 & -- & -- & -- & -- & - & -- \\
\hline 37N29W27CBBC3 & $08-12-91$ & 21.0 & 14.0 & -- & -- & -- & -- & -- & -- \\
\hline \multirow[t]{3}{*}{$37 \mathrm{~N} 29 \mathrm{~W} 34 \mathrm{ACA}$} & $07-19-90$ & 22.0 & 14.5 & 0.30 & -- & -- & -- & -- & -- \\
\hline & $08-06-91$ & 33.0 & 14.5 & -- & -- & - & -- & -- & -- \\
\hline & $09-12-94$ & 34.0 & 14.5 & -- & 678 & 7.0 & 78 & 144 & 144 \\
\hline
\end{tabular}


Table 11. Selected water-quality data from observation wells and private wells completed in the Ogallala aquifer sampled by the U.S. Geological Survey for the nitrate studies-Continued

\begin{tabular}{|c|c|c|c|c|c|c|c|c|}
\hline Local number & Date & $\begin{array}{c}\text { Calcium, } \\
\text { dissolved } \\
\text { (mg/L } \\
\text { as Ca) } \\
(00915) \\
\end{array}$ & $\begin{array}{c}\text { Magne- } \\
\text { sium, } \\
\text { dissolved } \\
(\mathrm{mg} / \mathrm{L} \\
\text { as Mg) } \\
(00925) \\
\end{array}$ & $\begin{array}{c}\text { Sodium, } \\
\text { dissolved } \\
(\mathrm{mg} / \mathrm{L} \\
\text { as } \mathrm{Na}) \\
(00930) \\
\end{array}$ & $\begin{array}{c}\text { Potassium, } \\
\text { dissolved } \\
\text { (mg/L } \\
\text { as K) } \\
(00935) \\
\end{array}$ & $\begin{array}{c}\text { Bicar- } \\
\text { bonate, } \\
\text { field, } \\
\text { dissolved, } \\
\text { IT } \\
\left(\mathrm{mgdl}^{2} \text { as }\right. \\
\left.\mathrm{HCO}_{3}\right) \\
(00453) \\
\end{array}$ & $\begin{array}{c}\text { Car- } \\
\text { bonate, } \\
\text { field, } \\
\text { dissolved, } \\
\text { IT }(\mathrm{mg} / \mathrm{L} \\
\left.\text { as } \mathrm{CO}_{3}\right) \\
(00452) \\
\end{array}$ & $\begin{array}{c}\text { Sulfate, } \\
\text { dissolved } \\
(\mathrm{mg} / \mathrm{L} \\
\text { as SO } \\
(00945) \\
\end{array}$ \\
\hline 35N29W 4BCCB3 & $08-12-91$ & -- & - & -- & -- & -- & -- & -- \\
\hline \multirow[t]{3}{*}{$36 \mathrm{~N} 29 \mathrm{~W} 8 \mathrm{~A}$} & 09-19-90 & 61 & 10 & 5.3 & 7.3 & -- & -- & 5.7 \\
\hline & $08-06-91$ & -- & -- & $-\cdot$ & -- & -- & -- & -- \\
\hline & $09-14-94$ & -- & -- & -- & -- & 0 & 204 & - \\
\hline 36N29W 8AABA & $09-14-94$ & -- & -- & -- & -- & 0 & 190 & -- \\
\hline 36N29W17AAAB & $09-13-94$ & -- & -- & -- & -- & 0 & 180 & -- \\
\hline 36N29W17AADD3 & $09-13-94$ & -- & -- & -- & -- & 0 & 268 & -- \\
\hline 36N29W17ACAA & $09-13-94$ & -- & -- & -- & -- & 0 & 237 & -- \\
\hline 36N29W17ACBC2 & $09-14-94$ & -. & -- & -. & -- & 0 & 453 & -- \\
\hline \multirow[t]{3}{*}{ 36N29W17ADBB } & $07-19-90$ & 110 & 13 & 8.9 & 9.9 & - & -- & 7.8 \\
\hline & $08-06-91$ & -- & -- & -- & -- & -- & -- & -- \\
\hline & $09-13-94$ & -- & -- & -- & -- & 0 & 211 & -- \\
\hline 36N29W18AADD & $09-12-94$ & - & -- & -- & -- & 0 & 182 & - \\
\hline \multirow[t]{2}{*}{36 N29W29ACAA } & $07-18-90$ & 35 & 5.3 & 37 & 9.5 & -- & -- & 16 \\
\hline & $08-06-91$ & -- & -- & -- & -- & -- & -- & -- \\
\hline $37 \mathrm{~N} 29 \mathrm{~W} 27 \mathrm{CBBC} 3$ & $08-12-91$ & -- & -- & -- & -- & -- & -- & -- \\
\hline \multirow[t]{3}{*}{$37 \mathrm{~N} 29 \mathrm{~W} 34 \mathrm{ACA}$} & $07-19-90$ & 120 & 4.3 & 3.9 & 8.8 & -- & -- & 28 \\
\hline & $08-06-91$ & -- & -- & -- & -- & -- & -- & -- \\
\hline & $09-12-94$ & -- & -- & -- & -- & 0 & 176 & -- \\
\hline
\end{tabular}


Table 11. Selected water-quality data from observation wells and private wells completed in the Ogallala aquifer sampled by the U.S. Geological Survey for the nitrate studies-Continued

\begin{tabular}{|c|c|c|c|c|c|c|c|c|}
\hline Local number & Date & $\begin{array}{c}\text { Chloride, } \\
\text { dissolved } \\
\text { (mg/L } \\
\text { as Cl) } \\
(00940)\end{array}$ & $\begin{array}{c}\text { Fluoride, } \\
\text { dissolved } \\
\text { ( } \mathrm{mg} / 1 \\
\text { as F) } \\
(00950)\end{array}$ & $\begin{array}{c}\text { Silica, } \\
\text { dissolved } \\
(\mathrm{mg} / \mathrm{L} \text { as } \\
\left.\mathrm{SiO}_{2}\right) \\
(00955)\end{array}$ & $\begin{array}{c}\text { Solids, } \\
\text { residue } \\
\text { at } 180 \\
\text { deg C, } \\
\text { dissolved } \\
\text { (mg/L) } \\
(70300)\end{array}$ & $\begin{array}{l}\text { Nitrogen, } \\
\text { ammonia, } \\
\text { dissolved } \\
\text { (mg/L } \\
\text { as } \mathrm{N}) \\
(00608)\end{array}$ & $\begin{array}{c}\text { Nitrogen, } \\
\text { nitrite, } \\
\text { dissolved } \\
\text { (mg/L as N) } \\
\text { (00613) }\end{array}$ & $\begin{array}{c}\text { Nitrogen, } \\
\mathrm{NO}_{2}+\mathrm{NO}_{3}, \\
\text { dissolved } \\
\text { (mg/L } \\
\text { as } \mathrm{N}) \\
(00631)\end{array}$ \\
\hline $35 \mathrm{~N} 29 \mathrm{~W} 4 \mathrm{BCCB} 3$ & $08-12-91$ & -- & $\overline{--}$ & -- & $\overline{--}$ & $<0.01$ & $<0.01$ & 31.0 \\
\hline \multirow[t]{3}{*}{$36 \mathrm{~N} 29 \mathrm{~W} 8 \mathrm{~A}$} & $09-19-90$ & 8.0 & 0.30 & 54 & 282 & -- & -- & 9.10 \\
\hline & $08-06-91$ & -- & -- & -- & -- & 0.02 & $<0.01$ & 8.10 \\
\hline & $09-14-94$ & 4.0 & -- & -- & -- & $<0.002$ & -- & 5.70 \\
\hline $36 \mathrm{~N} 29 \mathrm{~W} 8 \mathrm{AABA}$ & $09-14-94$ & 3.0 & -- & -- & -- & 0.003 & -- & 7.80 \\
\hline 36N29W17AAAB & $09-13-94$ & 1.3 & -- & -- & -- & $<0.002$ & -- & 3.10 \\
\hline 36N29W17AADD3 & $09-13-94$ & 1.7 & -- & -- & -- & $<0.002$ & -- & 6.70 \\
\hline 36N29W17ACAA & $09-13-94$ & 6.9 & -- & -- & -- & $<0.002$ & -- & 23.0 \\
\hline $36 \mathrm{~N} 29 \mathrm{~W} 17 \mathrm{ACBC} 2$ & $09-14-94$ & 9.6 & -- & -- & -- & $<0.002$ & - & 5.50 \\
\hline \multirow[t]{3}{*}{ 36N29W17ADBB } & $07-19-90$ & 21 & 0.30 & 54 & 492 & -- & -- & 29.0 \\
\hline & $08-06-91$ & -- & -- & -- & -- & 0.02 & $<0.01$ & 25.0 \\
\hline & $09-13-94$ & 12 & -- & -- & -- & $<0.002$ & -- & 23.0 \\
\hline 36N29W18AADD & $09-12-94$ & 2.4 & -- & -- & -- & $<0.002$ & -- & 1.50 \\
\hline \multirow[t]{2}{*}{ 36N29W29ACAA } & $07-18-90$ & 2.8 & 0.50 & 69 & 279 & -- & -- & 1.10 \\
\hline & $08-06-91$ & -- & -- & -- & -- & 0.01 & $<0.01$ & 1.10 \\
\hline $37 \mathrm{~N} 29 \mathrm{~W} 27 \mathrm{CBBC} 3$ & $08-12-91$ & - & -- & -- & -- & $<0.01$ & $<0.01$ & 3.70 \\
\hline \multirow[t]{3}{*}{$37 \mathrm{~N} 29 \mathrm{~W} 34 \mathrm{ACA}$} & $07-19-90$ & 28 & 0.30 & 55 & 598 & -- & -- & 44.0 \\
\hline & $08-06-91$ & - & -- & -- & -- & 0.02 & $<0.01$ & 30.0 \\
\hline & $09-12-94$ & 18 & -- & -- & -- & $<0.002$ & -- & 23.0 \\
\hline
\end{tabular}


Table 11. Selected water-quality data from observation wells and private wells completed in the Ogallala aquifer sampled by the U.S. Geological Survey for the nitrate studies-Continued

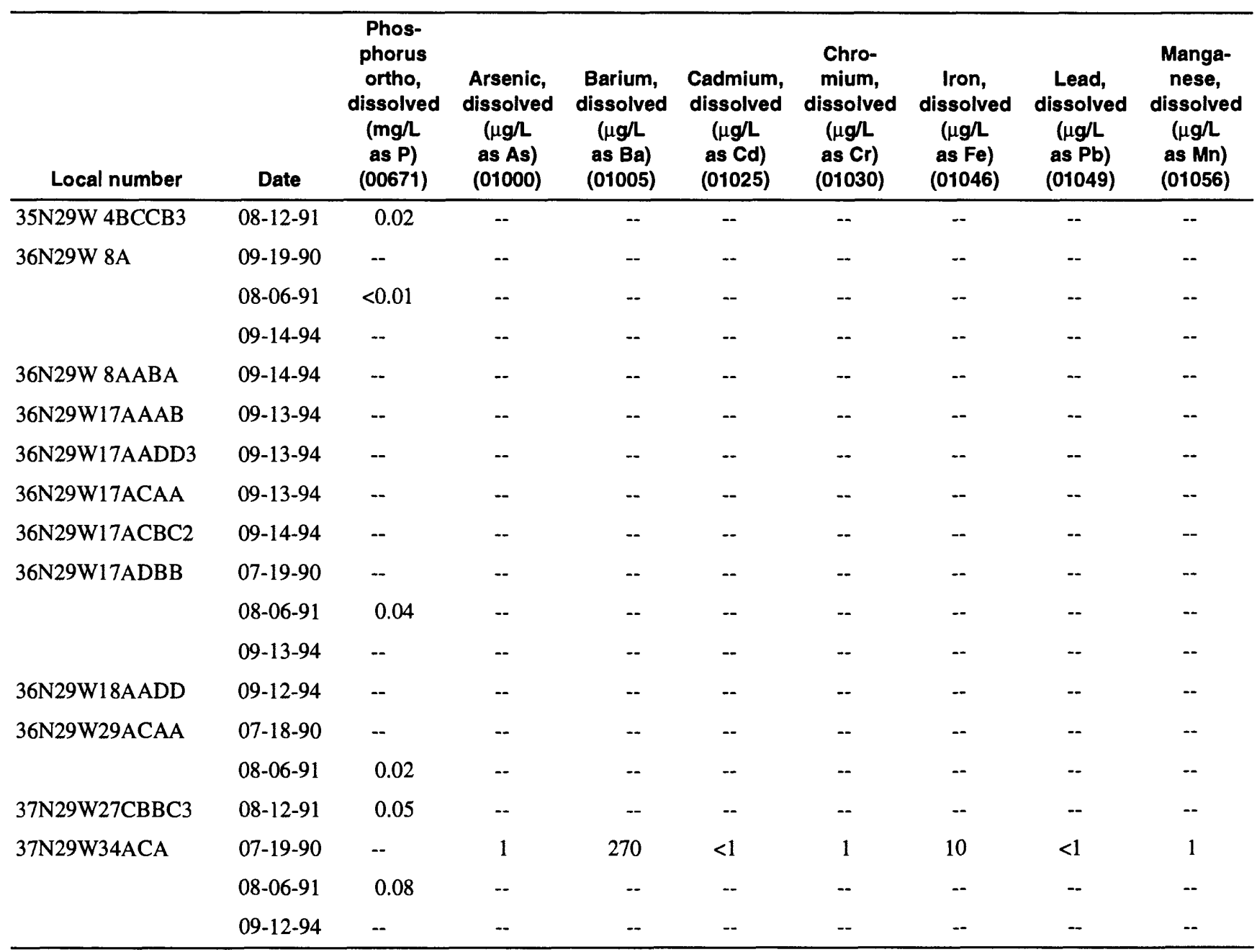


Table 11. Selected water-quality data from observation wells and private wells completed in the Ogallala aquifer sampled by the U.S. Geological Survey for the nitrate studies-Continued

\begin{tabular}{|c|c|c|c|c|c|c|c|c|c|}
\hline Local number & Date & $\begin{array}{c}\text { Mercury, } \\
\text { dissolved } \\
(\mu \mathrm{g} / \mathrm{L} \\
\text { as } \mathrm{Hg}) \\
(71890)\end{array}$ & $\begin{array}{c}\text { Selenium, } \\
\text { dissolved } \\
(\mu g / L \\
\text { as Se) } \\
(01145)\end{array}$ & $\begin{array}{c}\text { Zinc, } \\
\text { dissolved } \\
(\mu g / L \\
\text { as Zn) } \\
(01090)\end{array}$ & $\begin{array}{l}\text { Methy- } \\
\text { lene blue } \\
\text { active } \\
\text { substance } \\
\text { (mg/L) } \\
(\mathbf{3 8 2 6 0 )}\end{array}$ & $\begin{array}{c}\mathrm{N}-15 / \mathrm{N}-14 \\
\text { stable } \\
\text { isotope } \\
\text { ratio } \\
\text { per mil } \\
(\mathbf{8 2 0 8 4 )}\end{array}$ & $\begin{array}{c}\text { Alachlor, } \\
\text { total, } \\
\text { recover } \\
(\mu g / L) \\
(77825)\end{array}$ & $\begin{array}{c}\text { Ametryne, } \\
\text { total } \\
(\mu \mathrm{g} / \mathrm{L}) \\
(\mathbf{8 2 1 8 4})\end{array}$ & $\begin{array}{c}\text { Atrazine, } \\
\text { unfltrd, } \\
\text { recover } \\
(\mu g / L) \\
(39630)\end{array}$ \\
\hline $35 \mathrm{~N} 29 \mathrm{~W} 4 \mathrm{BCCB} 3$ & $08-12-91$ & -- & - & - & -- & 10.60 & $\cdots$ & -- & - \\
\hline \multirow[t]{3}{*}{$36 \mathrm{~N} 29 \mathrm{~W} 8 \mathrm{~A}$} & $09-19-90$ & -- & -- & -- & - & -- & - & - & -- \\
\hline & $08-06-91$ & -- & -- & -- & -- & 7.30 & - & -- & -- \\
\hline & $09-14-94$ & -- & -- & -- & 0.03 & -- & - & -- & -- \\
\hline $36 \mathrm{~N} 29 \mathrm{~W} 8 \mathrm{AABA}$ & $09-14-94$ & -- & -- & -- & $<0.02$ & -- & - & -- & -- \\
\hline 36N29W17AAAB & $09-13-94$ & -- & -- & -- & 0.03 & -- & - & -. & - \\
\hline 36N29W17AADD3 & $09-13-94$ & -- & -- & -- & $<0.02$ & -- & - & -- & -- \\
\hline 36N29W17ACAA & $09-13-94$ & -- & -- & -- & 0.02 & -- & - & -- & -- \\
\hline $36 \mathrm{~N} 29 \mathrm{~W} 17 \mathrm{ACBC} 2$ & $09-14-94$ & -- & -- & -- & $<0.02$ & -- & - & -- & -- \\
\hline \multirow[t]{3}{*}{ 36N29W17ADBB } & $07-19-90$ & -- & -- & -- & -- & -- & -- & -- & -- \\
\hline & $08-06-91$ & -- & -- & -- & -- & 7.90 & - & -- & -. \\
\hline & $09-13-94$ & -- & -- & -. & $<0.02$ & -- & - & -- & -- \\
\hline 36N29W18AADD & $09-12-94$ & - & -. & -- & 0.04 & - & - & -- & -- \\
\hline \multirow[t]{2}{*}{$36 \mathrm{~N} 29 \mathrm{~W} 29 \mathrm{ACAA}$} & $07-18-90$ & - & -- & -- & -- & -- & -- & -- & -- \\
\hline & $08-06-91$ & -- & -- & -- & - & 3.70 & -- & -- & -- \\
\hline $37 \mathrm{~N} 29 \mathrm{~W} 27 \mathrm{CBBC} 3$ & $08-12-91$ & -- & -- & -- & -- & 1.40 & -- & -- & -- \\
\hline \multirow[t]{3}{*}{$37 \mathrm{~N} 29 \mathrm{~W} 34 \mathrm{ACA}$} & $07-19-90$ & $<0.1$ & 1 & 54 & -- & -- & $<0.10$ & $<0.10$ & $<0.1$ \\
\hline & $08-06-91$ &.- & -- & -- & -- & 11.80 & -- &.- & -- \\
\hline & $09-12-94$ & -- & -- & -- & 0.03 & -- & -- & -- & -- \\
\hline
\end{tabular}


Table 11. Selected water-quality data from observation wells and private wells completed in the Ogallala aquifer sampled by the U.S. Geological Survey for the nitrate studies-Continued

\begin{tabular}{|c|c|c|c|c|c|c|c|c|c|}
\hline Local number & Date & $\begin{array}{c}\text { Cyan- } \\
\text { azine, } \\
\text { total } \\
(\mu g / L) \\
(81757)\end{array}$ & $\begin{array}{c}\text { Dicamba } \\
\text { (Mediben) } \\
\text { (Banvel D), } \\
\text { total } \\
\left(\mu g^{\prime} L\right) \\
(82052)\end{array}$ & $\begin{array}{c}2,4,5-T, \\
\text { total } \\
(\mu g / L) \\
(39740)\end{array}$ & $\begin{array}{c}2,4-D, \\
\text { total } \\
(\mu g / L) \\
(39730)\end{array}$ & $\begin{array}{c}2,4-D P, \\
\text { total } \\
(\mu g / L) \\
(82183)\end{array}$ & $\begin{array}{c}\text { Metrl- } \\
\text { buzin, } \\
\text { whole, } \\
\text { total. } \\
\text { recover } \\
(\mu g / L) \\
(82611)\end{array}$ & $\begin{array}{l}\text { Metola- } \\
\text { chlor, } \\
\text { whole total } \\
\text { recover } \\
(\mathbf{8 2 6 1 2})\end{array}$ & $\begin{array}{c}\text { Picloram } \\
\text { (Tordon) } \\
\text { (Amdon), } \\
\text { total } \\
(\mu g / L) \\
(39720)\end{array}$ \\
\hline $35 \mathrm{~N} 29 \mathrm{~W} 4 \mathrm{BCCB} 3$ & $08-12-91$ & - & -- & - & -- & - & - & - & -- \\
\hline \multirow[t]{3}{*}{$36 \mathrm{~N} 29 \mathrm{~W} 8 \mathrm{~A}$} & $09-19-90$ & - & - & -- &.- & - & -- & -- & -- \\
\hline & $08-06-91$ & -- & -- & -- & -- & -- & -- & -- & -- \\
\hline & $09-14-94$ & -- & -- & -- & -- & -- & -- & -- & -- \\
\hline 36N29W 8AABA & $09-14-94$ & -- & -- & -- & -- & -- & -- & -- & -- \\
\hline $36 \mathrm{~N} 29 \mathrm{~W} 17 \mathrm{AAAB}$ & $09-13-94$ & -- & -- & -- & -- & -- & -- & -- & -- \\
\hline 36N29W17AADD3 & $09-13-94$ & -- & -- & -- & -- & -- & -- & -- & -- \\
\hline $36 \mathrm{~N} 29 \mathrm{~W} 17 \mathrm{ACAA}$ & $09-13-94$ & -- & -- & -- & -- & -- & -- & -. & -- \\
\hline $36 \mathrm{~N} 29 \mathrm{~W} 17 \mathrm{ACBC} 2$ & $09-14-94$ & -- & -- & -- & -- & -- & -- & -- & -- \\
\hline \multirow[t]{3}{*}{ 36N29W17ADBB } & $07-19-90$ & -- & -- & -- & -- & -- & -- & -- & -- \\
\hline & $08-06-91$ & -- & -- & -- & -- & -- & -- & -- & -- \\
\hline & $09-13-94$ & -- & -- & -- & -- & -. & -- & - & -- \\
\hline 36N29W18AADD & $09-12-94$ & -- & -. & -. & -- & -- & -- & -- & -- \\
\hline \multirow[t]{2}{*}{ 36N29W29ACAA } & $07-18-90$ & -- & -- & -- & -- & -- & -- & -- & -- \\
\hline & $08-06-91$ & -- & -- & -- & -- & -- & -- & -- & -- \\
\hline $37 \mathrm{~N} 29 \mathrm{~W} 27 \mathrm{CBBC} 3$ & $08-12-91$ & -- & -- & -- & -- & -- & -- & -- & -- \\
\hline \multirow[t]{3}{*}{ 37N29W34ACA } & $07-19-90$ & $<0.10$ & $<0.01$ & $<0.01$ & 0.05 & $<0.01$ & $<0.10$ & $<0.10$ & $<0.01$ \\
\hline & 08-06-91 & -- & -- & -- & -- & -- & -- & -- & -- \\
\hline & $09-12-94$ & -- & -- & -- & -. & -- & -- & -- & -- \\
\hline
\end{tabular}


Table 11. Selected water-quality data from observation wells and private wells completed in the Ogallala aquifer sampled by the U.S. Geological Survey for the nitrate studies-Continued

\begin{tabular}{|c|c|c|c|c|c|c|c|c|}
\hline Local number & Date & $\begin{array}{c}\text { Prome- } \\
\text { tone, } \\
\text { total } \\
(\mu g / L) \\
(39056)\end{array}$ & $\begin{array}{c}\text { Prome- } \\
\text { tryne, } \\
\text { total } \\
(\mu g / L) \\
(39057)\end{array}$ & $\begin{array}{c}\text { Propazine, } \\
\text { total } \\
(\mu g / L) \\
(39024)\end{array}$ & $\begin{array}{c}\text { Silvex, } \\
\text { total } \\
(\mu g / L) \\
(39760)\end{array}$ & $\begin{array}{c}\text { Simazine, } \\
\text { total } \\
(\mu \mathrm{g} / \mathrm{L}) \\
(39055)\end{array}$ & $\begin{array}{c}\text { Simetryne, } \\
\text { total } \\
(\mu g / L) \\
(39054)\end{array}$ & $\begin{array}{c}\text { Trifluralin, } \\
\text { total, } \\
\text { recover } \\
(\mu g / L) \\
(39030)\end{array}$ \\
\hline 35N29W 4BCCB3 & $08-12-91$ & -- & -- & - & -- & -- & -- & -- \\
\hline \multirow[t]{3}{*}{ 36N29W 8A } & $09-19-90$ & -- & -- & -- & -- & -- & -- & -- \\
\hline & $08-06-91$ & -- & -- & -- & -- & -- & -- & -- \\
\hline & $09-14-94$ & -- & -- &.- & - & -. & -- & -- \\
\hline $36 \mathrm{~N} 29 \mathrm{~W} 8 \mathrm{AABA}$ & $09-14-94$ & -- & -- & -- & -- & -- & -- & -- \\
\hline 36N29W17AAAB & $09-13-94$ & -- & -- & -- & -- & -- & -- & -- \\
\hline 36N29W17AADD3 & $09-13-94$ & -- & -- & -- & -- & -- & -- & -- \\
\hline 36N29W17ACAA & $09-13-94$ & -- & -- & -- & -- & -- & -- & -- \\
\hline 36N29W17ACBC2 & $09-14-94$ & -- & -- & -- & -- & - & - & -- \\
\hline \multirow[t]{3}{*}{ 36N29W17ADBB } & $07-19-90$ & -- & -- & -- & -- & -- & -- & -- \\
\hline & $08-06-91$ & -- & -- & -- & -- & -- & -- & -- \\
\hline & $09-13-94$ & -- & -- & -- & -- & -- & -- & -- \\
\hline 36N29W18AADD & $09-12-94$ & -- & -- & -- & -- & -- & -- & -- \\
\hline \multirow[t]{2}{*}{ 36N29W29ACAA } & $07-18-90$ & -- & -- & - & -- & -- & -- & -- \\
\hline & $08-06-91$ & -- & -- & -- & -- & $-\cdot$ & -- & -- \\
\hline $37 \mathrm{~N} 29 \mathrm{~W} 27 \mathrm{CBBC} 3$ & $08-12-91$ & -- & -- & -- & -- & -- & -- & -- \\
\hline \multirow[t]{3}{*}{$37 \mathrm{~N} 29 \mathrm{~W} 34 \mathrm{ACA}$} & $07-19-90$ & $<0.10$ & $<0.10$ & $<0.10$ & $<0.01$ & $<0.10$ & $<0.10$ & $<0.10$ \\
\hline & $08-06-91$ & -- & - & -- & -- & -- & -- & - \\
\hline & $09-12-94$ & -- & -- & -- & -. & -- & -- & -. \\
\hline
\end{tabular}

${ }^{1}$ Laboratory value is given because parameter was not measured in the field 
Table 12. Quality-assurance results of blank sample collected during the nitrate study

[mg/L, milligrams per liter; $<$, less than]

\begin{tabular}{|c|c|c|c|c|c|c|}
\hline Station number & Local number & Date & $\begin{array}{c}\text { Chloride, } \\
\text { dissolved } \\
\text { (mg/L } \\
\text { as Cl) } \\
(00940)\end{array}$ & $\begin{array}{c}\text { Nitrogen, } \\
\text { ammonia, } \\
\text { dissolved } \\
\text { (mg/L } \\
\text { as } \mathrm{N} \text { ) } \\
(00608)\end{array}$ & $\begin{array}{c}\text { Nitrogen, } \\
\mathrm{NO}_{2}+\mathrm{NO}_{3}, \\
\text { dissolved } \\
\text { (mg/L as } \mathrm{N}) \\
(00631)\end{array}$ & $\begin{array}{c}\text { Methylene } \\
\text { blue active } \\
\text { substance } \\
(\mathbf{m g} / \mathrm{L}) \\
(\mathbf{3 8 2 6 0 )}\end{array}$ \\
\hline 430603100460501 & 36N29W18AADD & $09-12-94$ & $<0.10$ & $<0.002$ & 0.054 & $<0.02$ \\
\hline
\end{tabular}

Table 13. Chemical analyses of field duplicates for nitrate study water samples [mg/L, milligrams per liter; <, less than]

\begin{tabular}{|c|c|c|c|c|c|c|c|}
\hline Station number & Local number & $\begin{array}{c}\text { Sample } \\
\text { type }\end{array}$ & Date & $\begin{array}{c}\text { Chloride, } \\
\text { dissolved } \\
\text { (mg/L } \\
\text { as Cl) } \\
(00940)\end{array}$ & $\begin{array}{c}\text { Nitrogen, } \\
\text { ammonia, } \\
\text { dissolved } \\
\text { (mg/L as } \mathrm{N}) \\
(00608)\end{array}$ & $\begin{array}{c}\text { Nitrogen, } \\
\mathrm{NO}_{2}+\mathrm{NO}_{3}, \\
\text { dissolved } \\
\text { (mg/L as } \mathrm{N}) \\
(00631)\end{array}$ & $\begin{array}{c}\text { Methylene } \\
\text { blue active } \\
\text { substance } \\
\text { (mg/L) } \\
(\mathbf{3 8 2 6 0 )}\end{array}$ \\
\hline \multirow[t]{2}{*}{430600100450801} & $36 \mathrm{~N} 29 \mathrm{~W} 17 \mathrm{ACAA}$ & Sample & $09-13-94$ & 6.9 & $<0.002$ & 23.0 & 0.02 \\
\hline & & Duplicate & $09-13-94$ & 6.9 & $<0.002$ & 23.0 & 0.03 \\
\hline
\end{tabular}


Table 14. Selected water-quality data from observation wells completed in alluvial aquifers sampled by the U.S. Geological Survey

$[\mu \mathrm{S} / \mathrm{cm}$, microsiemens per centimeter; $\mathrm{mg} / \mathrm{L}$, milligrams per liter; $\mathrm{mm}$, millimeter; $\mathrm{mL}$, milliliter; $\mu \mathrm{g} / \mathrm{L}$, micrograms per liter; pci/L, picocuries per liter; $\operatorname{deg}$ C, degrees Celsius; dis, dissolved; FET, fixed end point titration; IT, incremental titration; NTU, nephelometric turbidity units; $\mu$ m-mf, micrometermembrane filter; cols, colonies; rec, recoverable; <, less than; --, no data]

\begin{tabular}{|c|c|c|c|c|c|c|c|c|}
\hline Station number & Local number & $\begin{array}{c}\text { Other } \\
\text { identifier }\end{array}$ & County & Date & $\begin{array}{c}\text { Depth of } \\
\text { well } \\
\text { (feet) } \\
\text { (72008) }\end{array}$ & $\begin{array}{c}\text { Water level } \\
\text { (feet) } \\
(72019)\end{array}$ & $\begin{array}{c}\text { Specific } \\
\text { conduct- } \\
\text { ance, } \\
\text { field } \\
(\mu S / \mathrm{cm}) \\
(00095)\end{array}$ & $\begin{array}{c}\text { pH, } \\
\text { field } \\
\text { (standard } \\
\text { units) } \\
(00400)\end{array}$ \\
\hline 435132101023001 & 2S26E20BCCB & R2-96-26 & Jones & $10-01-96$ & 23.0 & 6.48 & 2,530 & 7.0 \\
\hline 432746100172201 & 40N25W 4CCDD & R2-96-32 & Mellette & 09-30-96 & 37.5 & 6.99 & 1,830 & 7.3 \\
\hline 434253100181301 & 43N25W 9CACA & R2-96-33 & Mellette & 09-30-96 & 22.0 & 13.49 & 1,720 & 7.4 \\
\hline 434141100523601 & $43 \mathrm{~N} 30 \mathrm{~W} 22 \mathrm{ABAB}$ & R2-96-23 & Mellette & $10-01-96$ & 107.0 & 94.25 & 422 & 7.3 \\
\hline 430715101031901 & $36 \mathrm{~N} 32 \mathrm{~W} 2 \mathrm{CDBD}$ & R2-93-23 & Todd & 09-07-94 & 44.0 & 9.47 & 229 & 7.7 \\
\hline
\end{tabular}

\begin{tabular}{|c|c|c|c|c|c|c|c|c|c|c|}
\hline $\begin{array}{l}\text { Temper- } \\
\text { ature, } \\
\text { air } \\
\text { (deg C) } \\
(00020)\end{array}$ & $\begin{array}{l}\text { Temper- } \\
\text { ature, } \\
\text { water } \\
\text { (deg C) } \\
\text { (00010) }\end{array}$ & $\begin{array}{c}\text { Turbidity } \\
\text { (NTU) } \\
\text { (00076) }\end{array}$ & $\begin{array}{c}\text { Baro- } \\
\text { metric } \\
\text { pressure } \\
(\mathrm{mm} \text { of } \\
\mathrm{Hg}) \\
(00025)\end{array}$ & $\begin{array}{c}\text { Oxygen, } \\
\text { dissolved } \\
(\mathrm{mg} / \mathrm{L}) \\
(00300)\end{array}$ & $\begin{array}{c}\text { Oxygen, } \\
\text { dissolved } \\
\text { (percent } \\
\text { satur- } \\
\text { ation) } \\
\text { (00301) }\end{array}$ & $\begin{array}{c}\text { Alkalinity, } \\
\text { dissolved, } \\
\text { IT, field } \\
\text { (mg/L as } \\
\mathrm{CaCO}_{3} \text { ) } \\
(39086)\end{array}$ & $\begin{array}{l}\text { Alkalinity, } \\
\text { dissolved, } \\
\text { FET, field } \\
\text { (mg/L as } \\
\left.\mathrm{CaCO}_{3}\right) \\
(00418)\end{array}$ & $\begin{array}{c}\text { Coliform, } \\
\text { fecal, } 0.7 \\
\mu \mathrm{m}-\mathrm{mf} \\
\text { (cols.J } \\
100 \mathrm{~mL}) \\
(31625)\end{array}$ & $\begin{array}{c}\text { Strep- } \\
\text { tococci, } \\
\text { fecal, } \\
\text { KF agar } \\
\text { (cols.J } \\
100 \mathrm{~mL} \text { ) } \\
(31673)\end{array}$ & $\begin{array}{c}\text { Calcium, } \\
\text { dissolved } \\
\text { (mg/L } \\
\text { as Ca) } \\
(00915)\end{array}$ \\
\hline 13.0 & 11.5 & -- & 710 & 0.6 & 6 & 355 & 355 & 0 & 0 & 240 \\
\hline 29.0 & 11.5 & .- & 7 & 0.1 & 1 & 257 & 259 & -- & -- & 270 \\
\hline 22.5 & 12.5 & -. & 721 & 0.6 & 6 & 288 & 291 & -- & -- & 110 \\
\hline 9.0 & 13.0 & -- & 699 & 11.2 & 116 & 173 & 173 & -- & -. & 58 \\
\hline 17.0 & 13.0 & 0.30 & 692 & 1.1 & 11 & 96 & 98 & 0 & 0 & 25 \\
\hline
\end{tabular}

\begin{tabular}{|c|c|c|c|c|c|c|c|c|c|}
\hline $\begin{array}{l}\text { Magne- } \\
\text { sium, } \\
\text { dissolved } \\
\text { (mg/L } \\
\text { as Mg) } \\
(00925)\end{array}$ & $\begin{array}{c}\text { Sodium, } \\
\text { dissolved } \\
\text { (mg/L } \\
\text { as Na) } \\
(00930)\end{array}$ & $\begin{array}{l}\text { Sodium, } \\
\text { percent } \\
\text { (00932) }\end{array}$ & $\begin{array}{l}\text { Sodium } \\
\text { adsorption } \\
\text { ratio } \\
(00931)\end{array}$ & $\begin{array}{l}\text { Potassium, } \\
\text { dissolved } \\
\text { (mg/L } \\
\text { as K) } \\
(00935)\end{array}$ & $\begin{array}{c}\text { Bicar- } \\
\text { bonate, } \\
\text { dissolved, } \\
\text { IT, field } \\
\text { (mg/L as } \\
\left.\mathrm{HCO}_{3}\right) \\
(00453)\end{array}$ & $\begin{array}{c}\text { Carbonate, } \\
\text { dissolved, } \\
\text { IT, field } \\
\text { (mg/L as } \\
\left.\mathrm{CO}_{3}\right) \\
(\mathbf{0 0 4 5 2 )}\end{array}$ & $\begin{array}{c}\text { Sulfate, } \\
\text { dissolved } \\
\text { (mg/L as } \\
\left.\mathrm{SO}_{4}\right) \\
(00945)\end{array}$ & $\begin{array}{c}\text { Chloride, } \\
\text { dissolved } \\
\text { (mg/L } \\
\text { as Cl) } \\
(00940)\end{array}$ & $\begin{array}{c}\text { Fluoride, } \\
\text { dissolved } \\
\text { (mg/L } \\
\text { as F) } \\
(00950)\end{array}$ \\
\hline 50 & 370 & 49 & 6 & 14 & 433 & 0 & 1,200 & 17 & 0.20 \\
\hline 54 & 72 & 15 & 1 & 16 & 314 & 0 & 810 & 22 & 0.20 \\
\hline 15 & 310 & 66 & 7 & 8.7 & 351 & 0 & 590 & 100 & 0.40 \\
\hline 9.3 & 8.1 & 8 & 0.3 & 7.5 & 211 & 0 & 22 & 4.3 & 0.20 \\
\hline 3.5 & 14 & 26 & 0.7 & 6.5 & 117 & 0 & 5.9 & 0.90 & 0.30 \\
\hline
\end{tabular}


Table 14. Selected water-quality data from observation wells completed in alluvial aquifers sampled by the U.S. Geological Survey-Continued

\begin{tabular}{|c|c|c|c|c|c|c|c|c|c|}
\hline $\begin{array}{c}\text { Silica, } \\
\text { dissolved } \\
(\mathrm{mg} / \mathrm{L} \text { as } \\
\left.\mathrm{SiO}_{2}\right) \\
(00955)\end{array}$ & $\begin{array}{c}\text { Solids, } \\
\text { residue } \\
\text { at } 180 \\
\text { deg C, } \\
\text { dissolved } \\
\text { (mg/L) } \\
(70300)\end{array}$ & $\begin{array}{l}\text { Nitrogen, } \\
\text { ammonia, } \\
\text { dissolved } \\
\text { (mg/L } \\
\text { as } N \text { ) } \\
(00608)\end{array}$ & $\begin{array}{l}\text { Nitrogen, } \\
\text { nitrite, } \\
\text { dissolved } \\
\text { (mg/L } \\
\text { as } N \text { ) } \\
(00613)\end{array}$ & $\begin{array}{c}\text { Nitrogen, } \\
\mathrm{NO}_{2}+\mathrm{NO}_{3} \\
\text { dissolved } \\
\text { (mg/L } \\
\text { as } \mathrm{N}) \\
(00631)\end{array}$ & $\begin{array}{c}\text { Phos- } \\
\text { phorus, } \\
\text { ortho, } \\
\text { dissolved } \\
\text { (mg/L } \\
\text { as P) } \\
(00671)\end{array}$ & $\begin{array}{c}\text { Arsenic, } \\
\text { dissolved } \\
(\mu \mathrm{g} / \mathrm{L} \\
\text { as As) } \\
(01000)\end{array}$ & $\begin{array}{c}\text { Boron, } \\
\text { dissolved } \\
(\mu \mathrm{g} / \mathrm{L} \\
\text { as B) } \\
(01020)\end{array}$ & $\begin{array}{c}\text { Iron, } \\
\text { dissolved } \\
(\mu \mathrm{g} / \mathrm{L} \\
\text { as Fe) } \\
(01046)\end{array}$ & $\begin{array}{c}\text { Manga- } \\
\text { nese, } \\
\text { dissolved } \\
(\mu g / L \\
\text { as } M n) \\
(01056)\end{array}$ \\
\hline 27 & 2,220 & 0.150 & $<0.010$ & $<0.050$ & 0.010 & 3 & 443 & 530 & 960 \\
\hline 37 & 1,540 & 0.080 & $<0.010$ & 0.700 & 0.070 & 3 & 191 & 230 & 250 \\
\hline 27 & 1,380 & $<0.020$ & 0.010 & 1.20 & 0.030 & $<1$ & 354 & $<3$ & 83 \\
\hline 39 & 273 & $<0.015$ & $<0.010$ & 4.60 & 0.120 & 7 & 38 & $<3$ & 2.0 \\
\hline 60 & 175 & 0.002 & 0.002 & 0.662 & 0.053 & 6 & 20 & $<3$ & 6 \\
\hline
\end{tabular}

\begin{tabular}{|c|c|c|c|c|c|c|c|c|}
\hline $\begin{array}{c}\text { Selenium, } \\
\text { dissolved } \\
(\mu g / \text { as } \mathrm{Se}) \\
(01145)\end{array}$ & $\begin{array}{c}\text { Gross alpha, } \\
\text { dissolved } \\
\text { ( } \mu \text { g/l as } \\
\text { natural } \\
\text { uranium) } \\
(80030)\end{array}$ & $\begin{array}{l}\text { Alpha, count, } \\
2 \text { sigma, } \\
\text { dissolved as } \\
\text { natural } \\
\text { uranium } \\
(\mu g / L) \\
(75986)\end{array}$ & $\begin{array}{c}\text { Alpha radio, } \\
\text { dissolved } \\
\text { as Th-230 } \\
\text { (pci/l) } \\
(04126)\end{array}$ & $\begin{array}{l}\text { Alpha count, } \\
2 \text { sigma, } \\
\text { dissolved } \\
\text { as Th-230 } \\
\text { (pci/l) } \\
(75987)\end{array}$ & $\begin{array}{c}\text { Gross beta, } \\
\text { dissolved } \\
\text { (pci/l } \\
\text { as Cs-137) } \\
(03515)\end{array}$ & $\begin{array}{c}\text { Beta, } \\
2 \text { sigma, } \\
\text { dissolved } \\
\text { as Cs-137 } \\
\text { (pci/l) } \\
(75989)\end{array}$ & $\begin{array}{c}\text { Gross beta, } \\
\text { dissolved } \\
\text { (pci/L as } \\
\text { Sr/Yt-90) } \\
(80050)\end{array}$ & $\begin{array}{c}\text { Beta, } \\
2 \text { sigma, } \\
\text { dissolved } \\
\text { as } \\
\text { Sr90/Y90 } \\
\text { (pci/L) } \\
\text { (75988) }\end{array}$ \\
\hline 4 & -- & -. & 17 & 2.6 & 39 & 18 & -- & -. \\
\hline 7 & -- & .. & 6.1 & 1.2 & 20 & 9.4 & -- & -- \\
\hline 3 & -- & -- & $<3.0$ & 0.62 & 12 & 3.5 & -- & -- \\
\hline$<1$ & 1.8 & 0.83 & 1.3 & 0.58 & 8.5 & 2.0 & 6.4 & 1.9 \\
\hline
\end{tabular}


Table 15. Selected water-quality data from observation wells completed in the Ogallala aquifer sampled by the U.S. Geological Survey

$[\mu \mathrm{S} / \mathrm{cm}$, microsiemens per centimeter; $\mathrm{mg} / \mathrm{L}$, milligrams per liter; $\mathrm{mm}$, millimeter; $\mathrm{mL}$, milliliter; $\mu \mathrm{g} / \mathrm{L}$, micrograms per liter; pci/L, picocuries per liter; $\operatorname{deg}$ C, degrees Celsius; FET, fixed end point titration; IT, incremental titration; NTU, nephelometric turbidity units; $\mu$ m-mf, micrometer-membrane filter; cols, colonies; rec, recoverable; <, less than; --, no data; $K$, results based on colony count outside the acceptance range (non-ideal colony count)]

\begin{tabular}{|c|c|c|c|c|c|c|c|c|}
\hline Station number & Local number & $\begin{array}{c}\text { Other } \\
\text { identifier }\end{array}$ & County & Date & $\begin{array}{c}\text { Depth of } \\
\text { well } \\
\text { (feet) } \\
\text { (72008) }\end{array}$ & $\begin{array}{c}\text { Water level } \\
\text { (feet) } \\
(72019)\end{array}$ & $\begin{array}{c}\text { Specific } \\
\text { conduct- } \\
\text { ance, } \\
\text { field } \\
(\mu \mathrm{S} / \mathrm{cm}) \\
(00095)\end{array}$ & $\begin{array}{c}\text { pH, } \\
\text { field } \\
\text { (standard } \\
\text { units) } \\
(\mathbf{0 0 4 0 0 )}\end{array}$ \\
\hline 425956101134502 & $35 \mathrm{~N} 33 \mathrm{~W} 20 \mathrm{ABCC} 2$ & R2-93-30 & Bennett & $09-08-94$ & 64.0 & 7.89 & 218 & 7.6 \\
\hline 425956101134503 & $35 \mathrm{~N} 33 \mathrm{~W} 20 \mathrm{ABCC} 3$ & R2-93-29 & Bennett & $09-08-94$ & 165.0 & 7.96 & 337 & 7.7 \\
\hline 430023100115602 & 35N25W13DADD2 & R20-93-54 & Todd & $08-17-94$ & 160.0 & 9.30 & 367 & 7.5 \\
\hline 430002100174801 & $35 \mathrm{~N} 25 \mathrm{~W} 20 \mathrm{BBBC}$ & R2-94-02 & Todd & $08-30-94$ & 46.5 & 4.93 & 395 & 7.4 \\
\hline 430126100222001 & $35 \mathrm{~N} 26 \mathrm{~W} 10 \mathrm{CBBA} 2$ & R2-94-05 & Todd & $08-31-94$ & 82.0 & 46.56 & 394 & 7.5 \\
\hline 430245100292801 & $35 \mathrm{~N} 27 \mathrm{~W} 3 \mathrm{BBBB}$ & R2-93-37 & Todd & $08-24-94$ & 47.0 & 11.58 & 385 & 7.4 \\
\hline 430055100362702 & 35N28W15BBBD2 & R2-93-36 & Todd & 08-31-94 & 76.0 & 11.53 & 368 & 7.4 \\
\hline 430156100411901 & 35N29W 2DDDD2 & R2-94-09 & Todd & $08-24-94$ & 37.0 & 8.99 & 840 & 7.1 \\
\hline 425957100445302 & $35 \mathrm{~N} 29 \mathrm{~W} 20 \mathrm{AADD} 2$ & R2-94-08 & Todd & $08-24-94$ & 106.0 & 81.24 & 603 & 7.3 \\
\hline 430153100521303 & 35N30W 5DDCC3 & R2-96-38 & Todd & $10-03-96$ & 108.0 & 21.30 & 356 & 7.6 \\
\hline 430120100574901 & $35 \mathrm{~N} 31 \mathrm{~W} 10 \mathrm{CBBC}$ & R2-93-31 & Todd & $09-07-94$ & 57.0 & 4.33 & 393 & 7.4 \\
\hline 430152101054803 & $35 \mathrm{~N} 32 \mathrm{~W} 9 \mathrm{BABB} 3$ & R2-93-22 & Todd & ${ }^{1} 09-20-94$ & 67.0 & 7.53 & 187 & 7.3 \\
\hline 430614100362503 & $36 \mathrm{~N} 28 \mathrm{~W} 15 \mathrm{BABB} 3$ & $R 2-96-42$ & Todd & $10-02-96$ & 75.3 & 24.61 & 283 & 7.4 \\
\hline 430522100411902 & 36N29W14DDDD2 & R2-94-12 & Todd & $08-17-94$ & 225.0 & 71.81 & 361 & 7.5 \\
\hline 430929101104203 & 37N33W26AAAA3 & R2-92-52 & Todd & $08-16-94$ & 236.0 & 77.38 & 364 & 7.5 \\
\hline
\end{tabular}


Table 15. Selected water-quality data from observation wells completed in the Ogallala aquifer sampled by the U.S. Geological Survey-Continued

\begin{tabular}{|c|c|c|c|c|c|c|c|c|c|}
\hline Local number & $\begin{array}{c}\text { Temper- } \\
\text { ature, } \\
\text { air } \\
\text { (deg C) } \\
(\mathbf{0 0 0 2 0}) \\
\end{array}$ & $\begin{array}{l}\text { Temper- } \\
\text { ature, } \\
\text { water } \\
\text { (deg C) } \\
(00010) \\
\end{array}$ & $\begin{array}{c}\text { Turbidity } \\
\text { (NTU) } \\
\text { (00076) }\end{array}$ & $\begin{array}{c}\text { Baro- } \\
\text { metric } \\
\text { pressure } \\
\text { (mm of } \mathrm{Hg}) \\
(00025) \\
\end{array}$ & $\begin{array}{c}\text { Oxygen, } \\
\text { dissolved } \\
(\mathrm{mg} / \mathrm{L}) \\
(00300)\end{array}$ & $\begin{array}{c}\text { Oxygen, } \\
\text { dissolved } \\
\text { (percent } \\
\text { satur- } \\
\text { ation) } \\
\text { (00301) } \\
\end{array}$ & $\begin{array}{c}\text { Alkalinity, } \\
\text { dissolved, } \\
\text { IT, field } \\
\text { (mg/L as } \\
\left.\mathrm{CaCO}_{3}\right) \\
(39086) \\
\end{array}$ & $\begin{array}{l}\text { Alkalinity, } \\
\text { dissolved, } \\
\text { FET, field } \\
\text { (mg/L as } \\
\left.\mathrm{CaCO}_{3}\right) \\
(00418) \\
\end{array}$ & $\begin{array}{c}\text { Coliform, } \\
\text { fecal, } \\
0.7 \mu \mathrm{m}-\mathrm{mf} \\
\text { (cols. } \\
100 \mathrm{~mL}) \\
(31625)\end{array}$ \\
\hline 35N33W20ABCC2 & 16.5 & 12.0 & 0.20 & 680 & 8.5 & 89 & 102 & 100 & 0 \\
\hline 35N33W20ABCC3 & 23.0 & 13.0 & 0.10 & 679 & 8.3 & 88 & 140 & 142 & -. \\
\hline 35N25W13DADD2 & 18.0 & 13.0 & 1.4 & 775 & 8.9 & 83 & 169 & 169 & $-\cdot$ \\
\hline $35 \mathrm{~N} 25 \mathrm{~W} 20 \mathrm{BBBC}$ & 16.0 & 12.0 & 0.10 & 772 & 6.3 & 57 & 185 & 180 & 0 \\
\hline $35 \mathrm{~N} 26 \mathrm{~W} 10 \mathrm{CBBA} 2$ & 15.5 & 12.5 & 0.10 & 700 & 9.7 & 99 & 155 & 155 & 0 \\
\hline $35 \mathrm{~N} 27 \mathrm{~W} 3 \mathrm{BBBB}$ & 33.0 & 12.0 & 0.70 & 767 & 8.9 & 83 & 184 & 184 & 0 \\
\hline 35N28W15BBBD2 & 11.0 & 11.0 & 0.20 & 696 & 5.8 & 57 & 176 & 176 & 0 \\
\hline 35N29W 2DDDD2 & 23.0 & 11.0 & 2.1 & 767 & 5.9 & 53 & 300 & 296 & -- \\
\hline 35N29W20AADD2 & 18.0 & 12.5 & 0.30 & 767 & 9.3 & 87 & 177 & 178 & -- \\
\hline 35N30W 5DDCC3 & 2.0 & 12.0 & -- & 701 & 3.4 & 35 & 168 & 168 & -- \\
\hline $35 \mathrm{~N} 31 \mathrm{~W} 10 \mathrm{CBBC}$ & 26.0 & 12.0 & 0.20 & 687 & 7.2 & 74 & 177 & 177 & 0 \\
\hline $35 \mathrm{~N} 32 \mathrm{~W} 9 \mathrm{BABB} 3$ & 18.0 & 12.5 & 0.30 & 684 & 0.1 & 1 & 84 & 84 & -- \\
\hline 36N28W15BABB3 & 2.0 & 11.5 & -- & 706 & 7.5 & 75 & 153 & 149 & 0 \\
\hline 36N29W14DDDD2 & 26.0 & 14.5 & 1.4 & 762 & 7.5 & 73 & 163 & 164 & -- \\
\hline 37N33W26AAAA3 & 36.0 & 14.5 & 0.10 & 752 & 8.4 & 84 & 162 & 163 & -- \\
\hline
\end{tabular}


Table 15. Selected water-quality data from observation wells completed in the Ogallala aquifer sampled by the U.S. Geological Survey-Continued

\begin{tabular}{|c|c|c|c|c|c|c|c|c|}
\hline Local number & 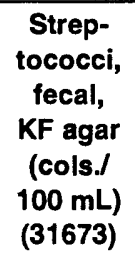 & $\begin{array}{c}\text { Calcium, } \\
\text { dissolved } \\
\text { (mg/L } \\
\text { as Ca) } \\
(00915)\end{array}$ & $\begin{array}{l}\text { Magne- } \\
\text { sium, } \\
\text { dissolved } \\
\text { (mg/L as } \\
\mathrm{Mg}) \\
(00925)\end{array}$ & $\begin{array}{c}\text { Sodium, } \\
\text { dissolved } \\
\text { (mg/L } \\
\text { as Na) } \\
(00930)\end{array}$ & $\begin{array}{l}\text { Sodium, } \\
\text { percent } \\
\text { (00932) }\end{array}$ & $\begin{array}{l}\text { Sodium } \\
\text { adsorption } \\
\text { ratio } \\
\text { (00931) }\end{array}$ & $\begin{array}{c}\text { Potassium, } \\
\text { dissolved } \\
\text { (mg/L as K) } \\
(00935)\end{array}$ & $\begin{array}{c}\text { Bicar- } \\
\text { bonate, } \\
\text { dissolved, } \\
\text { IT, field } \\
\left(\mathrm{mg}^{2} \mathrm{~h} \text { as }\right. \\
\left.\mathrm{HCO}_{3}\right) \\
(00453)\end{array}$ \\
\hline $35 \mathrm{~N} 33 \mathrm{~W} 20 \mathrm{ABCC} 2$ & 0 & 34 & 3.7 & 2.8 & 5 & 0.1 & 4.6 & 124 \\
\hline 35N33W20ABCC3 & -- & 39 & 6.8 & 16 & 20 & 0.6 & 7.7 & 171 \\
\hline 35N25W13DADD2 & -- & 43 & 8.5 & 17 & 19 & 0.6 & 8.9 & 206 \\
\hline $35 \mathrm{~N} 25 \mathrm{~W} 20 \mathrm{BBBC}$ & 0 & 41 & 7.7 & 22 & 25 & 0.8 & 10 & 226 \\
\hline $35 \mathrm{~N} 26 \mathrm{~W} 10 \mathrm{CBBA} 2$ & 0 & 53 & 8.8 & 3.7 & 4 & 0.1 & 9.7 & 189 \\
\hline $35 \mathrm{~N} 27 \mathrm{~W}$ 3BBBB & 0 & 55 & 6.3 & 9.8 & 11 & 0.3 & 11 & 225 \\
\hline 35N28W15BBBD2 & 0 & 53 & 6.8 & 3.7 & 4 & 0.1 & 11 & 215 \\
\hline 35N29W 2DDDD2 & -- & 100 & 7.6 & 45 & 24 & 1 & 24 & 366 \\
\hline 35N29W20AADD2 & -- & 99 & 6.5 & 3.4 & 3 & 0.1 & 8.9 & 216 \\
\hline 35N30W 5DDCC3 & -- & 46 & 6.9 & 10 & 12 & 0.4 & 8.8 & 205 \\
\hline $35 \mathrm{~N} 31 \mathrm{~W} 10 \mathrm{CBBC}$ & 0 & 50 & 7.0 & 17 & 18 & 0.6 & 7.7 & 216 \\
\hline $35 \mathrm{~N} 32 \mathrm{~W} 9 \mathrm{BABB} 3$ & -- & 24 & 2.5 & 5.5 & 13 & 0.3 & 6.3 & 103 \\
\hline 36N28W15BABB3 & $\mathrm{K} 2$ & 46 & 7.3 & 2.4 & 3 & 0.1 & 7.3 & 187 \\
\hline 36N29W14DDDD2 & -- & 42 & 11 & 11 & 13 & 0.4 & 10 & 199 \\
\hline 37N33W26AAAA3 & -- & 36 & 7.4 & 27 & 31 & 1 & 9.9 & 198 \\
\hline
\end{tabular}


Table 15. Selected water-quality data from observation wells completed in the Ogallala aquifer sampled by the U.S. Geological Survey-Continued

\begin{tabular}{|c|c|c|c|c|c|c|c|c|}
\hline Local number & 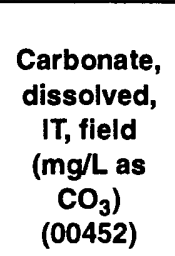 & $\begin{array}{c}\text { Sulfate, } \\
\text { dissolved } \\
\text { (mg/L as } \\
\left.\mathrm{SO}_{4}\right) \\
(00945)\end{array}$ & $\begin{array}{l}\text { Chloride, } \\
\text { dissolved } \\
\text { (mg/L } \\
\text { as Cl) } \\
(00940)\end{array}$ & $\begin{array}{c}\text { Fluoride, } \\
\text { dissolved } \\
\text { (mg/L as F) } \\
\text { (00950) }\end{array}$ & $\begin{array}{c}\text { Silica, } \\
\text { dissolved } \\
\text { (mg/L } \\
\left.\text { as } \mathrm{SiO}_{2}\right) \\
(00955)\end{array}$ & $\begin{array}{c}\text { Solids, } \\
\text { residue } \\
\text { at } 180 \\
\text { deg C, } \\
\text { dissolved } \\
(\mathrm{mg} / \mathrm{L}) \\
(70300)\end{array}$ & $\begin{array}{c}\text { Nitrogen, } \\
\text { ammonia, } \\
\text { dissolved } \\
\text { (mg/L } \\
\text { as N) } \\
(00608)\end{array}$ & $\begin{array}{c}\text { Nitrogen, } \\
\text { nitrite, } \\
\text { dissolved } \\
\text { (mg/L } \\
\text { as } \mathrm{N}) \\
(00613)\end{array}$ \\
\hline $35 \mathrm{~N} 33 \mathrm{~W} 20 \mathrm{ABCC} 2$ & 0 & 1.2 & 0.80 & 0.20 & 59 & 148 & $<0.002$ & $<0.001$ \\
\hline $35 \mathrm{~N} 33 \mathrm{~W} 20 \mathrm{ABCC} 3$ & 0 & 17 & 0.80 & 0.50 & 64 & 248 & $<0.002$ & $<0.001$ \\
\hline 35N25W13DADD2 & 0 & 7.2 & 0.80 & 0.50 & 65 & 265 & -- & -- \\
\hline $35 \mathrm{~N} 25 \mathrm{~W} 20 \mathrm{BBBC}$ & 0 & 11 & 1.6 & 0.50 & 69 & 262 & 0.002 & $<0.001$ \\
\hline $35 \mathrm{~N} 26 \mathrm{~W} 10 \mathrm{CBBA} 2$ & 0 & 7.0 & 2.3 & 0.30 & 62 & 275 & $<0.002$ & $<0.001$ \\
\hline $35 \mathrm{~N} 27 \mathrm{~W}$ 3BBBB & 0 & 4.3 & 2.8 & 0.40 & 61 & 260 & $<0.002$ & $<0.001$ \\
\hline 35N28W15BBBD2 & 0 & 3.4 & 1.0 & 0.30 & 59 & 251 & $<0.002$ & $<0.001$ \\
\hline 35N29W 2DDDD2 & 0 & 46 & 2.3 & 0.60 & 50 & 538 & 0.011 & 0.001 \\
\hline $35 \mathrm{~N} 29 \mathrm{~W} 20 \mathrm{AADD} 2$ & 0 & 11 & 2.0 & 0.20 & 55 & 439 & 0.003 & 0.001 \\
\hline 35N30W 5DDCC3 & 0 & 5.2 & 1.3 & 0.40 & 57 & 252 & $<0.015$ & $<0.010$ \\
\hline $35 \mathrm{~N} 31 \mathrm{~W} 10 \mathrm{CBBC}$ & 0 & 15 & 0.70 & 0.30 & 62 & 277 & $<0.002$ & $<0.001$ \\
\hline 35N32W 9BABB3 & 0 & 4.3 & 0.50 & 0.30 & 62 & 154 & 0.011 & 0.001 \\
\hline 36N28W15BABB3 & 0 & 3.2 & 2.9 & 0.30 & 59 & 228 & $<0.015$ & $<0.010$ \\
\hline 36N29W14DDDD2 & 0 & 8.6 & 1.7 & 0.50 & 64 & 253 & $<0.002$ & 0.002 \\
\hline 37N33W26AAAA3 & 0 & 14 & 0.90 & 0.40 & 63 & 216 & $<0.002$ & 0.002 \\
\hline
\end{tabular}


Table 15. Selected water-quality data from observation wells completed in the Ogallala aquifer sampled by the U.S. Geological Survey-Continued

\begin{tabular}{|c|c|c|c|c|c|c|c|c|}
\hline Local number & $\begin{array}{c}\text { Nitrogen, } \\
\mathrm{NO}_{2}+\mathrm{NO}_{3} \\
\text { dissolved } \\
\text { (mg/L } \\
\text { as N) } \\
(00631)\end{array}$ & $\begin{array}{l}\text { Phos- } \\
\text { phorus, } \\
\text { ortho, } \\
\text { dissolved } \\
\text { (mg/L } \\
\text { as P) } \\
\text { (00671) }\end{array}$ & $\begin{array}{c}\text { Arsenic, } \\
\text { dissolved } \\
(\mu \mathrm{g} / \mathrm{L} \\
\text { as As) } \\
(01000)\end{array}$ & $\begin{array}{c}\text { Boron, } \\
\text { dissolved } \\
(\mu \mathrm{g} / \mathrm{L} \\
\text { as B) } \\
(01020)\end{array}$ & $\begin{array}{c}\text { Iron, } \\
\text { dissolved } \\
(\mu \mathrm{g} / \mathrm{L} \\
\text { as Fe) } \\
(01046)\end{array}$ & $\begin{array}{c}\text { Manga- } \\
\text { nese, } \\
\text { dissolved } \\
(\mu \mathrm{g} / \mathrm{L} \\
\text { as } \mathrm{Mn}) \\
(01056)\end{array}$ & $\begin{array}{c}\text { Selenium, } \\
\text { dissolved } \\
\text { ( } \mu \mathrm{g} / \mathrm{L} \\
\text { as Se) } \\
(01145)\end{array}$ & $\begin{array}{c}\text { Gross } \\
\text { alpha, } \\
\text { dissolved } \\
\text { ( } \mu \text { g/L as } \\
\text { natural } \\
\text { uranium) } \\
(80030)\end{array}$ \\
\hline $35 \mathrm{~N} 33 \mathrm{~W} 20 \mathrm{ABCC} 2$ & 0.804 & 0.692 & 3 & 10 & $<3$ & $<1$ & $<1$ & 0.7 \\
\hline $35 \mathrm{~N} 33 \mathrm{~W} 20 \mathrm{ABCC} 3$ & 1.90 & 0.020 & 15 & 40 & $<3$ & $<1$ & $<1$ & 3.6 \\
\hline 35N25W13DADD2 & -- & -- & 8 & 40 & $<3$ & $<1$ & $<1$ & 3.4 \\
\hline $35 \mathrm{~N} 25 \mathrm{~W} 20 \mathrm{BBBC}$ & 0.979 & 0.012 & 12 & 50 & $<3$ & $<1$ & $<1$ & 6.1 \\
\hline 35N26W10CBBA2 & 7.30 & 0.044 & 2 & 20 & $<3$ & $<1$ & $<1$ & 2.6 \\
\hline 35N27W 3BBBB & 1.50 & 0.057 & 4 & 30 & $<3$ & 4 & $<1$ & 3.1 \\
\hline 35N28W15BBBD2 & 0.935 & 0.123 & 2 & $<10$ & $<3$ & 1 & $<1$ & 4.7 \\
\hline 35N29W 2DDDD2 & 18.0 & 0.233 & 7 & 70 & $<3$ & $<1$ & 3 & 97 \\
\hline 35N29W20AADD2 & 26.0 & 0.096 & 2 & 10 & $<3$ & $<1$ & 1 & 3.4 \\
\hline 35N30W 5DDCC3 & 1.30 & 0.030 & 8 & 18 & $<3.0$ & 1.0 & $<1$ & - \\
\hline $35 \mathrm{~N} 31 \mathrm{~W} 10 \mathrm{CBBC}$ & 1.60 & 0.041 & 8 & 50 & 8 & 4 & $<1$ & 4.8 \\
\hline $35 \mathrm{~N} 32 \mathrm{~W} 9 \mathrm{BABB} 3$ & 0.124 & 0.202 & 9 & 30 & 130 & 90 & $<1$ & $<0.6$ \\
\hline 36N28W15BABB3 & 2.50 & 0.100 & 2 & 20 & $<3.0$ & $<1.0$ & 1 & -- \\
\hline 36N29W14DDDD2 & 0.744 & 0.478 & 8 & 30 & 12 & 2 & $<1$ & 3.4 \\
\hline 37N33W26AAAA3 & 0.744 & 0.021 & 8 & 50 & 5 & $<1$ & $<1$ & 4.9 \\
\hline
\end{tabular}


Table 15. Selected water-quality data from observation wells completed in the Ogallala aquifer sampled by the U.S. Geological Survey-Continued

\begin{tabular}{|c|c|c|c|c|c|c|c|c|}
\hline Local number & $\begin{array}{c}\text { Alpha, } \\
\text { count, } \\
2 \text { sigma, } \\
\text { dissolved } \\
\text { as natural } \\
\text { uranium } \\
(\mu g / L) \\
(75986)\end{array}$ & $\begin{array}{l}\text { Alpha radio, } \\
\text { dissolved } \\
\text { as Th-230 } \\
\text { (pci/L) } \\
(04126)\end{array}$ & $\begin{array}{c}\text { Alpha } \\
\text { count, } \\
2 \text { sigma, } \\
\text { dissolved } \\
\text { as Th-230 } \\
\text { (pci/l) } \\
\text { (75987) }\end{array}$ & $\begin{array}{c}\text { Gross beta, } \\
\text { dissolved } \\
\text { (pci/L as } \\
\text { Cs-137) } \\
(03515)\end{array}$ & $\begin{array}{c}\text { Beta, } \\
2 \text { sigma, } \\
\text { dissolved } \\
\text { as Cs-137 } \\
\text { (pci/L) } \\
(75989)\end{array}$ & $\begin{array}{c}\text { Gross beta, } \\
\text { dissolved } \\
\text { (pci/L as } \\
\text { Sr/Yt-90) } \\
(80050)\end{array}$ & $\begin{array}{c}\text { Beta, } \\
2 \text { sigma, } \\
\text { dissolved } \\
\text { as } \\
\text { Sr90/Y90 } \\
\text { (pci/L) } \\
(75988)\end{array}$ & $\begin{array}{c}\text { Triazine } \\
\text { screen } \\
\text { (ELISA) } \\
\text { whole, rec, } \\
\text { as atrazine } \\
(\mu \mathrm{g} / \mathrm{L}) \\
(\mathbf{3 4 7 5 7 )}\end{array}$ \\
\hline $35 \mathrm{~N} 33 \mathrm{~W} 20 \mathrm{ABCC} 2$ & 0.55 & $<0.6$ & 0.35 & 5.2 & 1.5 & 4.0 & 0.87 & $<1$ \\
\hline $35 \mathrm{~N} 33 \mathrm{~W} 20 \mathrm{ABCC} 3$ & 1.2 & 2.6 & 0.83 & 9.7 & 2.2 & 7.0 & 1.2 & -- \\
\hline 35N25W13DADD2 & 1.2 & 2.1 & 0.77 & 12 & 2.0 & 8.8 & 1.5 & -- \\
\hline $35 \mathrm{~N} 25 \mathrm{~W} 20 \mathrm{BBBC}$ & 1.6 & 4.5 & 1.2 & 16 & 2.5 & 12 & 1.9 & $<1$ \\
\hline 35N26W10CBBA2 & 0.95 & 1.8 & 0.65 & 11 & 2.0 & 8.4 & 1.5 & $<1$ \\
\hline 35N27W 3BBBB & 1.1 & 2.2 & 0.75 & 14 & 2.2 & 10 & 1.7 & $<1$ \\
\hline 35N28W15BBBD2 & 1.4 & 3.3 & 0.97 & 12 & 2.1 & 9.3 & 1.5 & $<1$ \\
\hline 35N29W 2DDDD2 & 11 & 71 & 8.2 & 72 & 9.0 & 54 & 6.8 & $<1$ \\
\hline 35N29W20AADD2 & 1.2 & 2.4 & 0.87 & 11 & 2.1 & 8.2 & 1.5 & $<1$ \\
\hline 35N30W 5DDCC3 & -- & $<3.0$ & 0.76 & 8.6 & 3.2 & -- & -. & -. \\
\hline $35 \mathrm{~N} 31 \mathrm{~W} 10 \mathrm{CBBC}$ & 1.4 & 3.0 & 0.95 & 9.4 & 1.7 & 7.0 & 1.3 & $<1$ \\
\hline 35N32W 9BABB3 & 0.47 & $<0.6$ & 0.33 & 6.6 & 1.3 & 5.4 & 1.0 & $<1$ \\
\hline 36N28W15BABB3 & -- & $<3.0$ & 0.81 & 10 & 3.4 & -- & -- & -- \\
\hline 36N29W14DDDD2 & 1.1 & 2.3 & 0.73 & 12 & 2.7 & 8.5 & 1.4 & -- \\
\hline 37N33W26AAAA3 & 1.3 & 3.3 & 0.95 & 13 & 2.1 & 9.5 & 1.6 & -- \\
\hline
\end{tabular}

${ }^{1}$ Well may be completed in the Ash Hollow aquifer. 
Table 16. Selected water-quality data from observation wells completed in the Arikaree aquifer sampled by the U.S.

Geological Survey

$[\mu \mathrm{S} / \mathrm{cm}$, microsiemens per centimeter; $\mathrm{mg} / \mathrm{L}$, milligrams per liter; $\mathrm{mm}$, millimeter; $\mathrm{mL}$, milliliter; $\mu \mathrm{g} / \mathrm{L}$, micrograms per liter; $\mathrm{pci} / \mathrm{L}$, picocuries per liter; $\operatorname{deg}$ C, degrees Celsius; FET, fixed end point titration; IT, incremental titration; NTU, nephelometric turbidity units; $\mu \mathrm{m}$-mf, micrometer-membrane filter; cols, colonies; rec, recoverable; <, less than; --, no data; K, results based on colony count outside the acceptance range (non-ideal colony count)]

\begin{tabular}{|c|c|c|c|c|c|c|c|c|}
\hline Station number & Local number & $\begin{array}{c}\text { Other } \\
\text { identifier }\end{array}$ & County & Date & $\begin{array}{c}\text { Depth of } \\
\text { well } \\
\text { (feet) } \\
\text { (72008) }\end{array}$ & $\begin{array}{c}\text { Water level } \\
\text { (feet) } \\
\text { (72019) }\end{array}$ & $\begin{array}{c}\text { Specific } \\
\text { conduct- } \\
\text { ance, } \\
\text { field } \\
(\mu \mathrm{S} / \mathrm{cm}) \\
(00095)\end{array}$ & $\begin{array}{c}\text { pH, } \\
\text { field } \\
\text { (standaro } \\
\text { units) } \\
(\mathbf{0 0 4 0 0 )}\end{array}$ \\
\hline 425956101134501 & $35 \mathrm{~N} 33 \mathrm{~W} 20 \mathrm{ABCC}$ & R2-93-28 & Bennett & $09-08-94$ & 580.0 & 7.24 & 664 & 8.0 \\
\hline 430605101135701 & $36 \mathrm{~N} 33 \mathrm{~W} 17 \mathrm{BACA}$ & R2-92-54 & Bennett & ${ }^{1} 09-20-94$ & 215.6 & -- & 509 & 7.7 \\
\hline 430605101135702 & $36 \mathrm{~N} 33 \mathrm{~W} 17 \mathrm{BACA} 2$ & R2-92-55 & Bennett & ${ }^{1} 09-20-94$ & 290.5 & -- & 592 & 8.0 \\
\hline 432742101115802 & 40N33W 3DDDC2 & R2-94-81 & Mellette & $10-03-96$ & 116.0 & 23.51 & 598 & 8.1 \\
\hline 430003100174802 & $35 \mathrm{~N} 25 \mathrm{~W} 20 \mathrm{BBBC} 3$ & R2-94-04 & Todd & $08-30-94$ & 120.0 & 4.20 & 399 & 7.5 \\
\hline 430126100221901 & $35 \mathrm{~N} 26 \mathrm{~W} 10 \mathrm{CBBA} 3$ & R2-94-06 & Todd & $08-31-94$ & 492.0 & 48.55 & 729 & 8.2 \\
\hline 430245100292701 & 35N27W 3BBBB4 & R2-94-07 & Todd & $08-24-94$ & 202.0 & 11.80 & 422 & 7.4 \\
\hline 430153100521302 & $35 \mathrm{~N} 30 \mathrm{~W}$ 5DDCC2 & R2-96-37 & Todd & $10-03-96$ & 323.0 & 20.69 & 500 & 7.6 \\
\hline 430153101054902 & $35 \mathrm{~N} 32 \mathrm{~W} 9 \mathrm{BABB} 5$ & $\mathrm{R} 2-92-56$ & Todd & $09-20-94$ & 452.0 & 8.62 & 505 & 7.6 \\
\hline 430614100244802 & $36 \mathrm{~N} 26 \mathrm{~W} 18 \mathrm{AAAA} 2$ & R2-96-44 & Todd & $10-02-96$ & 232.0 & 10.82 & 695 & 8.1 \\
\hline 430614100362502 & $36 \mathrm{~N} 28 \mathrm{~W} 15 \mathrm{BABB} 2$ & R2-96-41 & Todd & $10-02-96$ & 432.0 & 24.95 & 460 & 7.6 \\
\hline 430613100544901 & $36 \mathrm{~N} 31 \mathrm{~W} 12 \mathrm{DCCD}$ & R2-96-39 & Todd & $10-02-96$ & 194.0 & 57.16 & 321 & 7.1 \\
\hline 431159100412102 & $37 \mathrm{~N} 28 \mathrm{~W} 7 \mathrm{BBBC} 2$ & R20-94-45 & Todd & $09-21-94$ & 243.0 & 78.83 & 355 & 7.5 \\
\hline 430922100410302 & $37 \mathrm{~N} 28 \mathrm{~W} 30 \mathrm{BBAA} 2$ & R2-94-14 & Todd & 08-15-94 & 320.5 & 70.42 & 399 & 7.4 \\
\hline 430929101104202 & 37N33W26AAAA2 & R2-92-51 & Todd & $08-16-94$ & 395.0 & 77.98 & 526 & 7.4 \\
\hline 431600100413203 & 38N29W13ADDC3 & R2-94-70 & Todd & $10-02-96$ & 112.5 & 6.65 & 365 & 7.1 \\
\hline 431903100282002 & 39N27W35AAAB2 & R20-93-46 & Todd & $08-23-94$ & 91.0 & 40.20 & 353 & 7.5 \\
\hline 431949100584602 & 39N31W27BBBB2 & R2-95-27 & Todd & $10-01-96$ & 95.0 & 59.95 & 354 & 7.5 \\
\hline
\end{tabular}


Table 16. Selected water-quality data from observation wells completed in the Arikaree aquifer sampled by the U.S. Geological Survey-Continued

\begin{tabular}{|c|c|c|c|c|c|c|c|c|c|}
\hline Local number & $\begin{array}{c}\text { Temper- } \\
\text { ature, } \\
\text { air } \\
\text { (deg C) } \\
\text { (00020) }\end{array}$ & $\begin{array}{c}\text { Temper- } \\
\text { ature, } \\
\text { water } \\
\text { (deg C) } \\
\text { (00010) }\end{array}$ & $\begin{array}{l}\text { Turbidity } \\
\text { (NTU) } \\
\text { (00076) }\end{array}$ & $\begin{array}{c}\text { Baro- } \\
\text { metric } \\
\text { pressure } \\
\text { (mm of Hg) } \\
(00025)\end{array}$ & $\begin{array}{c}\text { Oxygen, } \\
\text { dissolved } \\
(\mathrm{mg} / \mathrm{L}) \\
(00300)\end{array}$ & $\begin{array}{c}\text { Oxygen, } \\
\text { dissolved } \\
\text { (percent } \\
\text { satur- } \\
\text { ation) } \\
\text { (00301) }\end{array}$ & $\begin{array}{c}\text { Alkalinity, } \\
\text { dissolved, } \\
\text { IT, field } \\
\text { (mg/l as } \\
\left.\mathrm{CaCO}_{3}\right) \\
(39086)\end{array}$ & $\begin{array}{l}\text { Alkalinity, } \\
\text { dissolved, } \\
\text { FET, fieid } \\
\text { (mg/h as } \\
\left.\mathrm{CaCO}_{3}\right) \\
(00418)\end{array}$ & 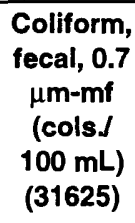 \\
\hline $35 \mathrm{~N} 33 \mathrm{~W} 20 \mathrm{ABCC}$ & 25.5 & 17.5 & 0.50 & 679 & 0.1 & 1 & 252 & 245 & - \\
\hline 36N33W17BACA & 30.0 & 17.0 & 0.80 & 684 & 3.0 & 34 & 197 & 197 & -- \\
\hline 36N33W17BACA2 & 28.0 & 18.0 & 0.20 & 684 & 0.5 & 6 & 213 & 213 & -- \\
\hline 40N33W 3DDDC2 & 20.0 & 14.5 & - & 705 & 5.2 & 55 & 272 & 267 & -- \\
\hline $35 \mathrm{~N} 25 \mathrm{~W} 20 \mathrm{BBBC} 3$ & 19.0 & 13.0 & 0.20 & 773 & 6.0 & 56 & 172 & 172 & -- \\
\hline $35 \mathrm{~N} 26 \mathrm{~W} 10 \mathrm{CBBA} 3$ & 12.5 & 17.0 & 0.50 & 700 & 0.4 & 4 & 282 & 277 & -- \\
\hline 35N27W 3BBBB4 & 36.0 & 15.0 & 0.20 & 766 & 7.4 & 72 & 192 & 191 & $-\cdot$ \\
\hline 35N30W 5DDCC2 & 2.0 & 15.5 & -- & 701 & 0.3 & 3 & 232 & 231 & -- \\
\hline 35N32W 9BABB5 & 19.0 & 16.0 & 38 & 684 & 0.7 & 7 & 219 & 209 & -. \\
\hline 36N26W18AAAA2 & 7.0 & 14.0 & -- & 710 & 0.1 & 1 & 280 & 280 & $-\cdot$ \\
\hline 36N28W15BABB2 & 2.0 & 18.0 & -- & 706 & 0.1 & 1 & 217 & 213 & -- \\
\hline 36N31W12DCCD & 7.0 & 13.5 & -- & 704 & 7.4 & 77 & 143 & 142 & -- \\
\hline $37 \mathrm{~N} 28 \mathrm{~W} 7 \mathrm{BBBC} 2$ & 14.0 & 13.5 & 0.50 & 701 & 7.0 & 73 & 178 & 178 & -- \\
\hline 37N28W30BBAA2 & 15.0 & 1.0 & 0.40 & 765 & 6.2 & 43 & 175 & 175 & -- \\
\hline 37N33W26AAAA2 & 33.0 & 16.0 & 0.20 & 754 & 6.4 & 66 & 189 & 189 & -- \\
\hline $38 \mathrm{~N} 29 \mathrm{~W} 13 \mathrm{ADDC} 3$ & 7.5 & 13.0 & -- & 711 & 6.9 & 70 & 180 & 181 & -- \\
\hline 39N27W35AAAB2 & 26.0 & 12.5 & 1.6 & 768 & 10.1 & 95 & 150 & 150 & 0 \\
\hline 39N31W27BBBB2 & 23.0 & 13.5 & -- & 696 & 8.8 & 93 & 168 & 168 & $\mathrm{~K} 1$ \\
\hline
\end{tabular}


Table 16. Selected water-quality data from observation wells completed in the Arikaree aquifer sampled by the U.S.

Geological Survey-Continued

\begin{tabular}{|c|c|c|c|c|c|c|c|c|}
\hline Local number & $\begin{array}{c}\text { Strep- } \\
\text { tococci, } \\
\text { fecal, } \\
\text { KF agar } \\
\text { (cols } J \\
100 \mathrm{~mL} \text { ) } \\
(31673)\end{array}$ & $\begin{array}{c}\text { Calcium, } \\
\text { dissolved } \\
\text { (mg/L } \\
\text { as Ca) } \\
(00915)\end{array}$ & $\begin{array}{l}\text { Magne- } \\
\text { sium, } \\
\text { dissolved } \\
\text { (mg/h } \\
\text { as } \mathrm{Mg}) \\
(00925)\end{array}$ & $\begin{array}{c}\text { Sodium, } \\
\text { dissolved } \\
\text { (mg/L } \\
\text { as Na) } \\
(00930)\end{array}$ & $\begin{array}{l}\text { Sodium, } \\
\text { percent } \\
(00932)\end{array}$ & $\begin{array}{c}\text { Sodium } \\
\text { adsorption } \\
\text { ratio } \\
\text { (00931) }\end{array}$ & $\begin{array}{l}\text { Potassium, } \\
\text { dissolved } \\
\text { (mg/L } \\
\text { as K) } \\
(00935)\end{array}$ & $\begin{array}{c}\text { Bicar- } \\
\text { bonate, } \\
\text { dissolved, } \\
\text { IT, field } \\
\left(\mathrm{mg}^{\prime} \mathrm{L} \text { as }\right. \\
\left.\mathrm{HCO}_{3}\right) \\
(00453)\end{array}$ \\
\hline 35N33W20ABCC & - & 9.5 & 0.61 & 130 & 88 & 11 & 9.9 & 308 \\
\hline 36N33W17BACA & -- & 5.2 & 0.33 & 110 & 90 & 13 & 9.8 & 240 \\
\hline 36N33W17BACA2 & - & 5.8 & 0.25 & 120 & 90 & 13 & 10 & 245 \\
\hline 40N33W 3DDDC2 & -- & 4.8 & 0.19 & 130 & 93 & 16 & 7.2 & 331 \\
\hline 35N25W20BBBC3 & -- & 41 & 7.3 & 21 & 24 & 0.8 & 10 & 210 \\
\hline 35N26W10CBBA3 & -- & 8.0 & 0.81 & 150 & 89 & 14 & 12 & 344 \\
\hline 35N27W 3BBBB4 & -- & 44 & 8.3 & 25 & 25 & 0.9 & 13 & 234 \\
\hline 35N30W 5DDCC2 & -- & 29 & 3.2 & 74 & 60 & 3 & 17 & 283 \\
\hline 35N32W 9BABB5 & -- & 25 & 2.9 & 75 & 64 & 4 & 15 & 262 \\
\hline $36 \mathrm{~N} 26 \mathrm{~W} 18 \mathrm{AAAA} 2$ & -- & 4.7 & 0.43 & 150 & 92 & 18 & 13 & 341 \\
\hline 36N28W15BABB2 & -- & 5.0 & 0.51 & 100 & 89 & 11 & 9.6 & 265 \\
\hline 36N31W12DCCD & -- & 34 & 6.0 & 21 & 28 & 0.9 & 7.4 & 174 \\
\hline $37 \mathrm{~N} 28 \mathrm{~W} 7 \mathrm{BBBC} 2$ & -- & 37 & 7.2 & 28 & 31 & 1 & 9.6 & 217 \\
\hline 37N28W30BBAA2 & -- & 41 & 7.0 & 31 & 32 & 1 & 11 & 214 \\
\hline 37N33W26AAAA2 & -- & 27 & 3.4 & 80 & 63 & 4 & 15 & 230 \\
\hline $38 \mathrm{~N} 29 \mathrm{~W} 13 \mathrm{ADDC} 3$ & -- & 44 & 6.0 & 20 & 23 & 0.8 & 9.9 & 220 \\
\hline $39 \mathrm{~N} 27 \mathrm{~W} 35 \mathrm{AAAB} 2$ & 0 & 52 & 8.1 & 4.1 & 5 & 0.1 & 7.0 & 183 \\
\hline 39N31W27BBBB2 & 0 & 31 & 2.7 & 41 & 47 & 2 & 8.3 & 205 \\
\hline
\end{tabular}


Table 16. Selected water-quality data from observation wells completed in the Arikaree aquifer sampled by the U.S. Geological Survey-Continued

\begin{tabular}{|c|c|c|c|c|c|c|c|c|}
\hline Local number & $\begin{array}{c}\text { Carbonate, } \\
\text { dissolved, } \\
\text { IT, field } \\
\left(\mathrm{mg}^{\prime} \mathrm{L} \text { as }\right. \\
\left.\mathrm{CO}_{3}\right) \\
(00452)\end{array}$ & $\begin{array}{c}\text { Sulfate, } \\
\text { dissolved } \\
\left(\mathrm{mg}^{\prime} \mathrm{L} \text { as }\right. \\
\left.\mathrm{SO}_{4}\right) \\
(00945)\end{array}$ & $\begin{array}{l}\text { Chloride, } \\
\text { dissolved } \\
\text { (mg/L } \\
\text { as Cl) } \\
(00940)\end{array}$ & $\begin{array}{c}\text { Fluoride, } \\
\text { dissolved } \\
\text { (mg/L } \\
\text { as F) } \\
(00950)\end{array}$ & $\begin{array}{c}\text { Silica, } \\
\text { dissolved } \\
(\mathrm{mg} / \mathrm{L} \text { as } \\
\left.\mathrm{SiO}_{2}\right) \\
(00955)\end{array}$ & $\begin{array}{l}\text { Solids, } \\
\text { residue } \\
\text { at } 180 \\
\text { deg C, } \\
\text { dissolved } \\
(\mathrm{mg} / \mathrm{L}) \\
(70300)\end{array}$ & $\begin{array}{c}\text { Nitrogen, } \\
\text { ammonia, } \\
\text { dissolved } \\
\text { (mg/L } \\
\text { as } N \text { ) } \\
(00608)\end{array}$ & $\begin{array}{c}\text { Nitrogen, } \\
\text { nitrite, } \\
\text { dissolved } \\
\text { (mg/h } \\
\text { as } \mathrm{N}) \\
(00613)\end{array}$ \\
\hline $35 \mathrm{~N} 33 \mathrm{~W} 20 \mathrm{ABCC}$ & 0 & 65 & 6.0 & 0.70 & 22 & 356 & 0.009 & 0.015 \\
\hline 36N33W17BACA & 0 & 37 & 2.6 & 0.40 & 80 & 370 & $<0.002$ & $<0.001$ \\
\hline 36N33W17BACA2 & 7 & 63 & 5.9 & 0.60 & 75 & 408 & $<0.002$ & $<0.001$ \\
\hline 40N33W 3DDDC2 & 0 & 22 & 4.5 & 0.20 & 60 & 398 & 0.050 & $<0.010$ \\
\hline $35 \mathrm{~N} 25 \mathrm{~W} 20 \mathrm{BBBC} 3$ & 0 & 11 & 1.7 & 0.50 & 69 & 266 & 0.002 & $<0.001$ \\
\hline $35 \mathrm{~N} 26 \mathrm{~W} 10 \mathrm{CBBA} 3$ & 0 & 74 & 2.8 & 1.8 & 71 & 505 & 0.084 & 0.003 \\
\hline 35N27W 3BBBB4 & 0 & 13 & 1.4 & 0.50 & 68 & 293 & $<0.002$ & 0.001 \\
\hline 35N30W 5DDCC2 & 0 & 47 & 1.1 & 0.50 & 68 & 388 & $<0.015$ & $<0.010$ \\
\hline 35N32W 9BABB5 & 0 & 39 & 1.3 & 0.40 & 77 & 351 & 0.245 & $<0.001$ \\
\hline 36N26W18AAAA2 & 0 & 62 & 4.4 & 1.5 & 61 & 480 & 0.020 & $<0.010$ \\
\hline 36N28W15BABB2 & 0 & 32 & 1.5 & 0.90 & 69 & 405 & $<0.015$ & 0.020 \\
\hline 36N31W12DCCD & 0 & 14 & 0.40 & 0.50 & 65 & 256 & $<0.015$ & $<0.010$ \\
\hline 37N28W 7BBBC2 & 0 & 9.3 & 1.6 & 0.40 & 62 & 260 & $<0.002$ & $<0.001$ \\
\hline 37N28W30BBAA2 & 0 & 9.4 & 1.7 & 0.50 & 73 & 275 & 0.021 & 0.003 \\
\hline 37N33W26AAAA2 & 0 & 60 & 3.1 & 0.40 & 77 & 374 & $<0.002$ & 0.002 \\
\hline 38N29W13ADDC3 & 0 & 9.0 & 1.4 & 0.40 & 67 & 285 & $<0.015$ & $<0.010$ \\
\hline 39N27W35AAAB2 & 0 & 7.7 & 2.1 & $<0.10$ & 59 & 262 & $<0.002$ & $<0.001$ \\
\hline 39N31W27BBBB2 & 0 & 7.2 & 3.9 & 0.30 & 65 & 256 & 0.020 & $<0.010$ \\
\hline
\end{tabular}


Table 16. Selected water-quality data from observation wells completed in the Arikaree aquifer sampled by the U.S. Geological Survey-Continued

\begin{tabular}{|c|c|c|c|c|c|c|c|c|}
\hline Local number & $\begin{array}{c}\text { Nitrogen, } \\
\mathrm{NO}_{2}+\mathrm{NO}_{3} \\
\text { dissolved } \\
\text { (mg/L } \\
\text { as N) } \\
(00631)\end{array}$ & $\begin{array}{c}\text { Phos- } \\
\text { phorus, } \\
\text { ortho, } \\
\text { dissolved } \\
\text { (mg/L } \\
\text { as P) } \\
\text { (00671) }\end{array}$ & $\begin{array}{c}\text { Arsenic, } \\
\text { dissolved } \\
(\mu \mathrm{g} / \mathrm{L} \\
\text { as As) } \\
(01000)\end{array}$ & $\begin{array}{c}\text { Boron, } \\
\text { dissolved } \\
(\mu \mathrm{g} / \mathrm{L} \\
\text { as B) } \\
(01020)\end{array}$ & $\begin{array}{c}\text { Iron, } \\
\text { dissolved } \\
(\mu \mathrm{g} / \mathrm{L} \\
\text { as Fe) } \\
(01046)\end{array}$ & $\begin{array}{c}\text { Manga- } \\
\text { nese, } \\
\text { dissolved } \\
(\mu g / L \\
\text { as Mn) } \\
(01056)\end{array}$ & $\begin{array}{c}\text { Selenium, } \\
\text { dissolved } \\
(\mu \mathrm{g} / \mathrm{L} \\
\text { as Se) } \\
(01145)\end{array}$ & $\begin{array}{c}\text { Gross } \\
\text { alpha, } \\
\text { dissolved } \\
(\mu g / L \text { as } \\
\text { natural } \\
\text { uranium) } \\
(80030)\end{array}$ \\
\hline $35 \mathrm{~N} 33 \mathrm{~W} 20 \mathrm{ABCC}$ & 1.50 & 0.117 & 36 & 360 & 45 & 21 & $<5$ & 9.2 \\
\hline 36N33W17BACA & 1.40 & 5.70 & 21 & 230 & 63 & 3 & 3 & 2.8 \\
\hline 36N33W17BACA2 & 0.548 & 0.007 & 23 & 310 & $<3$ & $<1$ & 1 & 6.4 \\
\hline $40 \mathrm{~N} 33 \mathrm{~W}$ 3DDDC2 & 0.580 & 0.180 & 3 & 120 & $<3.0$ & $<1.0$ & 2 & - \\
\hline $35 \mathrm{~N} 25 \mathrm{~W} 20 \mathrm{BBBC} 3$ & 1.00 & 0.041 & 12 & 50 & $<3$ & $<1$ & $<1$ & 6.4 \\
\hline $35 \mathrm{~N} 26 \mathrm{~W} 10 \mathrm{CBBA} 3$ & 0.975 & 0.489 & 110 & 340 & $<3$ & 14 & $<1$ & 4.4 \\
\hline $35 \mathrm{~N} 27 \mathrm{~W}$ 3BBBB4 & 1.40 & 0.213 & 18 & 60 & $<3$ & 5 & $<1$ & 8.2 \\
\hline 35N30W 5DDCC2 & 0.550 & 0.470 & 13 & 105 & 7.0 & 13 & 1 & -- \\
\hline $35 \mathrm{~N} 32 \mathrm{~W} 9 \mathrm{BABB} 5$ & $<0.005$ & 0.121 & 14 & 150 & 130 & 86 & $<1$ & 9.4 \\
\hline 36N26W18AAAA2 & $<0.050$ & 0.600 & 58 & 254 & 3.0 & 32 & $<1$ & -- \\
\hline 36N28W15BABB2 & 0.950 & 1.10 & 20 & 174 & 56 & 43 & 10 & -- \\
\hline 36N31W12DCCD & 1.80 & 0.680 & 16 & 43 & 7.0 & 8.0 & $<1$ & -- \\
\hline $37 \mathrm{~N} 28 \mathrm{~W} 7 \mathrm{BBBC} 2$ & 1.50 & 0.360 & 7 & 30 & $<3$ & $<1$ & $<1$ & 5.6 \\
\hline 37N28W30BBAA2 & 0.827 & 0.143 & 13 & 60 & 8 & 1 & 1 & 5.9 \\
\hline $37 \mathrm{~N} 33 \mathrm{~W} 26 \mathrm{AAAA} 2$ & 0.829 & 0.028 & 11 & 130 & $<3$ & 14 & 2 & 11 \\
\hline $38 \mathrm{~N} 29 \mathrm{~W} 13 \mathrm{ADDC} 3$ & 1.10 & $<0.010$ & 7 & 52 & $<3.0$ & $<1.0$ & $<1$ & - \\
\hline 39N27W35AAAB2 & 4.00 & 0.007 & 4 & 30 & $<3$ & $<1$ & 1 & 2.8 \\
\hline 39N31W27BBBB2 & 0.520 & 0.050 & 2 & 78 & $<3.0$ & $<1.0$ & $<1$ & -- \\
\hline
\end{tabular}


Table 16. Selected water-quality data from observation wells completed in the Arikaree aquifer sampled by the U.S. Geological Survey-Continued

\begin{tabular}{|c|c|c|c|c|c|c|c|c|}
\hline Local number & $\begin{array}{c}\text { Alpha, } \\
\text { count, } \\
2 \text { sigma, } \\
\text { dissolved } \\
\text { as natural } \\
\text { uranium } \\
(\mu \mathrm{g} / \mathrm{L}) \\
(75986)\end{array}$ & $\begin{array}{l}\text { Alpha radio, } \\
\text { dissolved } \\
\text { as Th-230 } \\
\text { (pci/l) } \\
(04126)\end{array}$ & $\begin{array}{c}\text { Alpha } \\
\text { count, } \\
2 \text { sigma, } \\
\text { dissolved } \\
\text { as Th-230 } \\
\text { (pcill) } \\
\text { (75987) }\end{array}$ & $\begin{array}{c}\text { Gross beta, } \\
\text { dissolved } \\
\text { (pci/L as } \\
\text { Cs-137) } \\
(03515)\end{array}$ & $\begin{array}{c}\text { Beta, } \\
2 \text { sigma, } \\
\text { dissolved } \\
\text { as Cs-137 } \\
(p c i / L) \\
(75989)\end{array}$ & $\begin{array}{c}\text { Gross beta, } \\
\text { dissolved } \\
\text { (pcill as } \\
\text { Sr/Yt-90) } \\
(80050)\end{array}$ & $\begin{array}{c}\text { Beta, } \\
2 \text { sigma, } \\
\text { dissolved } \\
\text { as } \\
\text { Sr90/Y90 } \\
\text { (pci/L) } \\
\text { (75988) }\end{array}$ & $\begin{array}{c}\text { Triazine } \\
\text { screen } \\
\text { (ELISA) } \\
\text { whole, rec, } \\
\text { as atrazine } \\
(\mu g / L) \\
(34757)\end{array}$ \\
\hline $35 \mathrm{~N} 33 \mathrm{~W} 20 \mathrm{ABCC}$ & 2.0 & 6.6 & 1.5 & 11 & 2.0 & 8.3 & 1.5 & -- \\
\hline 36N33W17BACA & 1.0 & 2.0 & 0.72 & 12 & 2.1 & 8.9 & 1.5 & -- \\
\hline $36 \mathrm{~N} 33 \mathrm{~W} 17 \mathrm{BACA} 2$ & 1.5 & 4.4 & 1.0 & 13 & 2.1 & 9.3 & 1.6 & -- \\
\hline 40N33W 3DDDC2 & -- & 15 & 2.2 & 22 & 5.9 & -- & -- & -- \\
\hline $35 \mathrm{~N} 25 \mathrm{~W} 20 \mathrm{BBBC} 3$ & 1.7 & 4.0 & 1.1 & 14 & 2.3 & 11 & 1.7 & $<1$ \\
\hline 35N26W10CBBA3 & 1.3 & 3.1 & 0.93 & 14 & 2.5 & 11 & 1.9 & -- \\
\hline 35N27W 3BBBB4 & 1.8 & 5.6 & 1.2 & 15 & 2.4 & 11 & 1.8 & -- \\
\hline 35N30W 5DDCC2 & -- & 8.8 & 1.5 & 28 & 6.7 & -- & -- & - \\
\hline 35N32W 9BABB5 & 2.0 & 6.6 & 1.4 & 14 & 2.3 & 11 & 1.7 & -- \\
\hline $36 \mathrm{~N} 26 \mathrm{~W} 18 \mathrm{AAAA} 2$ & -- & $<3.0$ & 0.70 & 22 & 6.0 & -- & -- & -- \\
\hline $36 \mathrm{~N} 28 \mathrm{~W} 15 \mathrm{BABB} 2$ & -- & $<3.0$ & 0.63 & 12 & 4.0 & -- & -- & -- \\
\hline 36N31W12DCCD & -- & $<3.0$ & 0.79 & 9.8 & 3.3 & -- & -- & -- \\
\hline $37 \mathrm{~N} 28 \mathrm{~W} 7 \mathrm{BBBC} 2$ & 1.5 & 3.9 & 1.1 & 9.9 & 2.3 & 7.3 & 1.3 & -- \\
\hline $37 \mathrm{~N} 28 \mathrm{~W} 30 \mathrm{BBAA} 2$ & 1.6 & 3.7 & 1.1 & 14 & 2.2 & 10 & 1.7 & -- \\
\hline 37N33W26AAAA2 & 2.1 & 7.5 & 1.5 & 19 & 2.9 & 14 & 2.1 & $-\cdot$ \\
\hline 38N29W13ADDC3 & -- & 5.8 & 1.2 & 13 & 3.8 & -- & -- & -- \\
\hline $39 \mathrm{~N} 27 \mathrm{~W} 35 \mathrm{AAAB} 2$ & 1.0 & 2.0 & 0.74 & 8.1 & 1.9 & 5.8 & 1.1 & $<1$ \\
\hline 39N31W27BBBB2 & -- & $<3.0$ & 0.76 & 11 & 3.2 & -- & -- & -- \\
\hline
\end{tabular}

${ }^{1}$ Indicates flowing well. 
Table 17. Selected water-quality data from observation wells completed in the White River aquifer sampled by the U.S. Geological Survey

$[\mu \mathrm{S} / \mathrm{cm}$, microsiemens per centimeter; $\mathrm{mg} / \mathrm{L}$, milligrams per liter; $\mathrm{mm}$, millimeter; $\mathrm{mL}$, milliliter; $\mu \mathrm{g} / \mathrm{L}$, micrograms per liter; pci/L, picocuries per liter; deg $\mathrm{C}$, degrees Celsius; FET, fixed end point titration; IT, incremental titration; NTU, nephelometric turbidity units; $\mu \mathrm{m}$-mf, micrometer-membrane filter; cols, colonies; rec, recoverable; <, less than; --, no data]

\begin{tabular}{|c|c|c|c|c|c|c|c|c|}
\hline Station number & Local number & $\begin{array}{c}\text { Other } \\
\text { identifier }\end{array}$ & County & Date & $\begin{array}{c}\text { Depth of } \\
\text { well } \\
\text { (feet) } \\
\text { (72008) }\end{array}$ & $\begin{array}{c}\text { Water level } \\
\text { (feet) } \\
\text { (72019) }\end{array}$ & $\begin{array}{c}\text { Specific } \\
\text { conduct- } \\
\text { ance, } \\
\text { field } \\
(\mu \mathrm{S} / \mathrm{cm}) \\
(00095)\end{array}$ & $\begin{array}{c}\mathrm{pH}, \\
\text { field } \\
\text { (standard } \\
\text { units) } \\
(00400)\end{array}$ \\
\hline 432555100591302 & 40N31W21AAAA2 & R2-92-47 & Mellette & $10-01-96$ & 81.2 & 57.73 & 628 & 7.4 \\
\hline 432649101081801 & $40 \mathrm{~N} 32 \mathrm{~W} 17 \mathrm{BBBB}$ & R2-94-76 & Mellette & $10-03-96$ & 552.5 & 14.12 & 2,540 & 7.7 \\
\hline 433248101121502 & 41N33W11ABDD2 & R2-96-19 & Mellette & $10-04-96$ & 265.0 & 135.61 & 3,280 & 7.9 \\
\hline 431203100135902 & $37 \mathrm{~N} 25 \mathrm{~W} 12 \mathrm{BBBB} 2$ & R20-93-49 & Todd & $08-30-94$ & 48.0 & 8.67 & 307 & 7.6 \\
\hline 431005100140001 & $37 \mathrm{~N} 25 \mathrm{~W} 24 \mathrm{BCBB}$ & R20-93-38 & Todd & 08-30-94 & 59.0 & 8.22 & 334 & 7.8 \\
\hline 431005100140002 & $37 \mathrm{~N} 25 \mathrm{~W} 24 \mathrm{BCBB} 2$ & R20-93-39 & Todd & $09-21-94$ & 107.0 & -- & 607 & 7.9 \\
\hline 431949100584601 & $39 \mathrm{~N} 31 \mathrm{~W} 27 \mathrm{BBBB}$ & R2-95-26 & Todd & $10-01-96$ & 565.0 & 85.06 & 1,670 & 7.7 \\
\hline 432320101044101 & $39 \mathrm{~N} 32 \mathrm{~W} 3 \mathrm{AAAA} 2$ & R2-92-45 & Todd & 08-16-94 & 526.5 & 74.89 & 1,520 & 8.0 \\
\hline 431811100125001 & 98N79W17DCBA & R20-93-43 & Tripp & $08-23-94$ & 37.0 & 13.87 & 360 & 7.4 \\
\hline
\end{tabular}

\begin{tabular}{|c|c|c|c|c|c|c|c|c|c|}
\hline Local number & $\begin{array}{l}\text { Temper- } \\
\text { ature, } \\
\text { air } \\
\text { (deg C) } \\
(00020)\end{array}$ & $\begin{array}{l}\text { Temper- } \\
\text { ature, } \\
\text { water } \\
\text { (deg C) } \\
(00010)\end{array}$ & $\begin{array}{c}\text { Turbidity } \\
\text { (NTU) } \\
\text { (00076) }\end{array}$ & $\begin{array}{c}\text { Baro- } \\
\text { metric } \\
\text { pressure } \\
(\mathrm{mm} \text { of } \mathrm{Hg}) \\
(00025)\end{array}$ & $\begin{array}{c}\text { Oxygen, } \\
\text { dissolved } \\
\text { (mg/L) } \\
(00300)\end{array}$ & $\begin{array}{c}\text { Oxygen, } \\
\text { dissolved } \\
\text { (percent } \\
\text { satur- } \\
\text { ation) } \\
\text { (00301) }\end{array}$ & $\begin{array}{c}\text { Alkalinity, } \\
\text { dissolved, } \\
\text { IT, field } \\
\text { (mg/L as } \\
\left.\mathrm{CaCO}_{3}\right) \\
(39086)\end{array}$ & $\begin{array}{l}\text { Alkalinity, } \\
\text { dissolved, } \\
\text { FET, field } \\
\left(\mathrm{mg}^{2} \mathrm{~L} \text { as }\right. \\
\left.\mathrm{CaCO}_{3}\right) \\
(00418)\end{array}$ & $\begin{array}{c}\text { Coliform, } \\
\text { fecal, } 0.7 \\
\mu \mathrm{m}-\mathrm{mf} \\
\text { (cols } J \\
100 \mathrm{~mL} \text { ) } \\
(31625)\end{array}$ \\
\hline 40N31W21AAAA2 & 20.5 & 13.5 & - & 694 & 9.8 & 103 & 152 & 152 & 0 \\
\hline 40N32W17BBBB & 15.0 & 19.0 & -- & 706 & 0.1 & 1 & 327 & 326 & -- \\
\hline 41N33W11ABDD2 & 8.0 & 16.0 & -- & 705 & 0.1 & 1 & 250 & 250 & -- \\
\hline 37N25W12BBBB2 & 26.5 & 13.0 & 1.1 & 777 & 8.9 & 83 & 132 & 132 & 0 \\
\hline $37 \mathrm{~N} 25 \mathrm{~W} 24 \mathrm{BCBB}$ & 26.0 & 12.5 & 0.10 & 699 & 6.4 & 66 & 147 & 147 & 0 \\
\hline 37N25W24BCBB2 & 9.5 & 12.5 & 1.0 & 704 & 0.1 & 1 & 267 & 260 & -- \\
\hline 39N31W27BBBB & 23.0 & 20.5 & -- & 696 & 0.1 & 1 & 428 & 421 & - \\
\hline 39N32W 3AAAA2 & 22.0 & 17.5 & 0.40 & 766 & 0.0 & 1 & 384 & 384 & -- \\
\hline 98N79W17DCBA & 23.0 & 13.0 & 0.20 & 773 & 6.7 & 62 & 164 & 165 & 0 \\
\hline
\end{tabular}


Table 17. Selected water-quality data from observation wells completed in the White River aquifer sampled by the U.S. Geological Survey-Continued

\begin{tabular}{|c|c|c|c|c|c|c|c|c|}
\hline Local number & 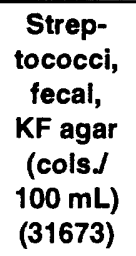 & $\begin{array}{c}\text { Calcium, } \\
\text { dissolved } \\
\text { (mg/L } \\
\text { as Ca) } \\
(00915)\end{array}$ & $\begin{array}{c}\text { Magne- } \\
\text { sium, } \\
\text { dissolved } \\
\text { (mgl as } \\
\text { Mg) } \\
(00925)\end{array}$ & $\begin{array}{c}\text { Sodium, } \\
\text { dissolved } \\
\text { (mgh } \\
\text { as Na) } \\
(00930)\end{array}$ & $\begin{array}{l}\text { Sodium, } \\
\text { percent } \\
\text { (00932) }\end{array}$ & $\begin{array}{c}\text { Sodium } \\
\text { adsorption } \\
\text { ratio } \\
\text { (00931) }\end{array}$ & $\begin{array}{c}\text { Potassium, } \\
\text { dissolved } \\
\text { (mg/L } \\
\text { as K) } \\
(00935)\end{array}$ & $\begin{array}{c}\text { Bicar- } \\
\text { bonate, } \\
\text { dissolved, } \\
\text { IT, field } \\
\left(\mathrm{mg}^{2} \mathrm{~L} \text { as }\right. \\
\left.\mathrm{HCO}_{3}\right) \\
(00453)\end{array}$ \\
\hline 40N31W21AAAA2 & 0 & 58 & 3.8 & 78 & 49 & 3 & 10 & 185 \\
\hline 40N32W17BBBB & -- & 19 & 1.1 & 520 & 94 & 31 & 12 & 399 \\
\hline 41N33W11 ABDD2 & -- & 24 & 1.5 & 650 & 95 & 35 & 8.4 & 305 \\
\hline $37 \mathrm{~N} 25 \mathrm{~W} 12 \mathrm{BBBB} 2$ & 0 & 40 & 6.9 & 6.0 & 9 & 0.2 & 7.2 & 161 \\
\hline $37 \mathrm{~N} 25 \mathrm{~W} 24 \mathrm{BCBB}$ & 0 & 12 & 1.2 & 52 & 71 & 4 & 9.1 & 179 \\
\hline 37N25W24BCBB2 & -- & 6.4 & 0.65 & 130 & 91 & 13 & 8.3 & 326 \\
\hline $39 \mathrm{~N} 31 \mathrm{~W} 27 \mathrm{BBBB}$ & -- & 10 & 0.60 & 350 & 95 & 29 & 11 & 523 \\
\hline 39N32W 3AAAA2 & -- & 8.9 & 0.43 & 330 & 95 & 29 & 8.4 & 469 \\
\hline 98N79W17DCBA & 0 & 51 & 7.3 & 9.3 & 11 & 0.3 & 8.4 & 200 \\
\hline
\end{tabular}

\begin{tabular}{|c|c|c|c|c|c|c|c|c|}
\hline Local number & $\begin{array}{c}\text { Carbonate, } \\
\text { dissolved, } \\
\text { IT, field } \\
(\mathrm{mg} / \mathrm{L} \\
\left.\text { as } \mathrm{CO}_{3}\right) \\
(00452)\end{array}$ & $\begin{array}{c}\text { Sulfate, } \\
\text { dissolved } \\
\text { (mg/L as } \\
\left.\mathrm{SO}_{4}\right) \\
(00945)\end{array}$ & $\begin{array}{c}\text { Chloride, } \\
\text { dissolved } \\
\text { (mg/L } \\
\text { as Cl) } \\
(00940)\end{array}$ & $\begin{array}{c}\text { Fluoride, } \\
\text { dissolved } \\
\text { (mg/L } \\
\text { as F) } \\
(00950)\end{array}$ & $\begin{array}{c}\text { Silica, } \\
\text { dissolved } \\
\text { (mg/l as } \\
\left.\mathrm{SiO}_{2}\right) \\
(00955)\end{array}$ & $\begin{array}{c}\text { Solids, } \\
\text { residue } \\
\text { at } 180 \\
\text { deg C, } \\
\text { dissolved } \\
(\mathrm{mg} / \mathrm{L}) \\
(70300)\end{array}$ & $\begin{array}{c}\text { Nitrogen, } \\
\text { ammonia, } \\
\text { dissolved } \\
\text { (mg/L } \\
\text { as } N) \\
(00608)\end{array}$ & $\begin{array}{c}\text { Nitrogen, } \\
\text { nitrite, } \\
\text { dissolved } \\
\text { (mg/L as N) } \\
\text { (00613) }\end{array}$ \\
\hline 40N31W21 AAAA2 & 0 & 170 & 5.9 & $<0.10$ & 63 & 502 & $<0.015$ & $<0.010$ \\
\hline 40N32W17BBBB & 0 & 0.50 & 610 & 1.2 & 13 & 1,400 & 0.550 & $<0.010$ \\
\hline 41N33W11 ABDD2 & 0 & 2.6 & 860 & 1.0 & 11 & 1,710 & 0.620 & $<0.010$ \\
\hline $37 \mathrm{~N} 25 \mathrm{~W} 12 \mathrm{BBBB} 2$ & 0 & 4.2 & 1.0 & 0.30 & 63 & 211 & 0.002 & $<0.001$ \\
\hline $37 \mathrm{~N} 25 \mathrm{~W} 24 \mathrm{BCBB}$ & 0 & 9.2 & 1.2 & 0.40 & 64 & 239 & 0.002 & $<0.001$ \\
\hline $37 \mathrm{~N} 25 \mathrm{~W} 24 \mathrm{BCBB} 2$ & 0 & 14 & 16 & 1.4 & 13 & 363 & 0.090 & 0.002 \\
\hline 39N31W27BBBB & 0 & 0.10 & 300 & 1.4 & 16 & 952 & 0.450 & $<0.010$ \\
\hline 39N32W 3AAAA2 & 0 & 18 & 230 & 1.7 & 17 & 856 & 0.275 & 0.007 \\
\hline 98N79W17DCBA & 0 & 3.2 & 0.70 & 0.20 & 61 & 253 & $<0.002$ & $<0.001$ \\
\hline
\end{tabular}


Table 17. Selected water-quality data from observation wells completed in the White River aquifer sampled by the U.S. Geological Survey-Continued

\begin{tabular}{|c|c|c|c|c|c|c|c|c|}
\hline Local number & $\begin{array}{c}\text { Nitrogen, } \\
\mathrm{NO}_{2}+\mathrm{NO}_{3} \\
\text { dissolved } \\
\text { (mg/L } \\
\text { as N) } \\
(00631)\end{array}$ & $\begin{array}{l}\text { Phos- } \\
\text { phorus, } \\
\text { ortho, } \\
\text { dissolved } \\
\text { (mg/L } \\
\text { as P) } \\
\text { (00671) }\end{array}$ & $\begin{array}{c}\text { Arsenic, } \\
\text { dissolved } \\
(\mu g / L \\
\text { as As) } \\
(01000)\end{array}$ & $\begin{array}{c}\text { Boron, } \\
\text { dissolved } \\
(\mu g / L \\
\text { as B) } \\
(01020)\end{array}$ & $\begin{array}{c}\text { Iron, } \\
\text { dissolved } \\
(\mu g / \mathrm{L} \\
\text { as Fe) } \\
(01046)\end{array}$ & $\begin{array}{c}\text { Manga- } \\
\text { nese, } \\
\text { dissolved } \\
(\mu g / L \\
\text { as Mn) } \\
(01056)\end{array}$ & $\begin{array}{c}\text { Selenium, } \\
\text { dissolved } \\
(\mu \mathrm{g} / \mathrm{L} \\
\text { as Se) } \\
(01145)\end{array}$ & $\begin{array}{c}\text { Gross } \\
\text { alpha, } \\
\text { dissolved } \\
(\mu g / L \text { as } \\
\text { natural } \\
\text { uranium) } \\
(80030)\end{array}$ \\
\hline 40N31W21AAAA2 & 2.80 & 0.260 & 3 & 92 & $<3.0$ & $<1.0$ & 5 & -- \\
\hline 40N32W17BBBB & $<0.050$ & 0.030 & 2 & 2,170 & 46 & 21 & $<1$ & -- \\
\hline 41N33W11ABDD2 & $<0.050$ & 0.760 & 4 & 2,160 & 11 & 24 & $<1$ & -- \\
\hline 37N25W12BBBB2 & 1.50 & 0.022 & 8 & 20 & $<3$ & $<1$ & $<1$ & 2.6 \\
\hline $37 \mathrm{~N} 25 \mathrm{~W} 24 \mathrm{BCBB}$ & 1.20 & 0.015 & 14 & 90 & $<3$ & $<1$ & 1 & 5.6 \\
\hline 37N25W24BCBB2 & $<0.005$ & 0.045 & $<1$ & 950 & 92 & 15 & $<1$ & $<0.6$ \\
\hline 39N31W27BBBB & $<0.050$ & 0.030 & 1 & 2,120 & 18 & 19 & $<1$ & -- \\
\hline 39N32W 3AAAA2 & $<0.005$ & 0.041 & 9 & 2,100 & 89 & 9 & $<1$ & 1.8 \\
\hline 98N79W17DCBA & 1.20 & 0.017 & 7 & 30 & 6 & 1 & $<1$ & 6.2 \\
\hline
\end{tabular}

\begin{tabular}{|c|c|c|c|c|c|c|c|c|}
\hline Local number & $\begin{array}{c}\text { Alpha, } \\
\text { count, } \\
2 \text { sigma, } \\
\text { dissolved } \\
\text { as natural } \\
\text { uranium } \\
(\mu g / L) \\
(75986)\end{array}$ & $\begin{array}{l}\text { Alpha radio, } \\
\text { dissolved } \\
\text { as Th-230 } \\
\text { (pci/L) } \\
(04126)\end{array}$ & $\begin{array}{c}\text { Alpha } \\
\text { count, } \\
2 \text { sigma, } \\
\text { dissolved } \\
\text { as Th-230 } \\
\text { (pcill) } \\
\text { (75987) }\end{array}$ & $\begin{array}{c}\text { Gross beta, } \\
\text { dissolved } \\
\text { (pci/L as } \\
\text { Cs-137) } \\
(03515)\end{array}$ & $\begin{array}{c}\text { Beta, } \\
2 \text { sigma, } \\
\text { dissolved } \\
\text { as Cs-137 } \\
\text { (pci/L) } \\
(75989)\end{array}$ & $\begin{array}{c}\text { Gross beta, } \\
\text { dissolved } \\
\text { (pci/L as } \\
\text { Sr/Yt-90) } \\
(80050)\end{array}$ & $\begin{array}{c}\text { Beta, } \\
2 \text { sigma, } \\
\text { dissolved } \\
\text { as } \\
\text { Sr90/Y9o } \\
\text { (pci/L) } \\
(75988)\end{array}$ & $\begin{array}{c}\text { Triazine } \\
\text { screen } \\
\text { (ELISA) } \\
\text { whole, rec, } \\
\text { as atrazine } \\
(\mu g / L) \\
(34757)\end{array}$ \\
\hline 40N31W21AAAA2 & -- & 5.3 & 1.1 & 18 & 5.0 & -- & -- & -- \\
\hline 40N32W17BBBB & -- & $<3.0$ & 0.46 & 21 & 11 & -- & -- & -- \\
\hline 41N33W11 ABDD2 & -- & $<3.0$ & 0.66 & 19 & 12 & -- & -- & -- \\
\hline 37N25W12BBBB2 & 1.0 & 1.8 & 0.72 & 8.7 & 2.1 & 6.3 & 1.1 & $<1$ \\
\hline $37 \mathrm{~N} 25 \mathrm{~W} 24 \mathrm{BCBB}$ & 1.5 & 3.9 & 1.1 & 12 & 2.6 & 8.8 & 1.4 & $<1$ \\
\hline $37 \mathrm{~N} 25 \mathrm{~W} 24 \mathrm{BCBB} 2$ & 0.50 & $<0.6$ & 0.35 & 9.9 & 1.8 & 7.4 & 1.4 & $<1$ \\
\hline 39N31W27BBBB & -- & 6.1 & 1.2 & 26 & 9.1 & -- & -- & -- \\
\hline 39N32W 3AAAA2 & 1.4 & 1.3 & 0.94 & 11 & 2.9 & 7.8 & 2.2 & -- \\
\hline 98N79W17DCBA & 1.6 & 4.3 & 1.1 & 11 & 2.5 & 7.8 & 1.3 & -- \\
\hline
\end{tabular}

\footnotetext{
${ }^{1}$ Indicates flowing well.
} 
Table 18. Quality-assurance results of blank samples collected during the observation well sampling program $[\mu \mathrm{S} / \mathrm{cm}$, microsiemens per centimeter; $\mathrm{mg} / \mathrm{L}$, milligrams per liter; $\mu \mathrm{g} / \mathrm{L}$, micrograms per liter; pci/L, picocuries per liter; deg $\mathrm{C}$, degrees Celsius; IT, incremental titration; NTU, nephelometric turbidity units; <, less than; --, no data]

\begin{tabular}{|c|c|c|c|c|c|c|c|}
\hline Station number & Sample type & Date & $\begin{array}{c}\text { Specific } \\
\text { conduct- } \\
\text { ance, } \\
\text { lab } \\
(\mu \mathrm{S} / \mathrm{cm}) \\
(00095)\end{array}$ & $\begin{array}{c}\text { pH, } \\
\text { lab } \\
\text { (standard } \\
\text { units) } \\
(00400)\end{array}$ & $\begin{array}{c}\text { Turbidity } \\
\text { (NTU) } \\
\text { (00076) }\end{array}$ & $\begin{array}{l}\text { Alkalinity, } \\
\text { dissolved, } \\
\text { IT, lab } \\
\left(\mathrm{mg}^{2} \mathrm{~L} \text { as }\right. \\
\left.\mathrm{CaCO}_{3}\right) \\
(90410)\end{array}$ & $\begin{array}{c}\text { Calcium, } \\
\text { dissolved } \\
\text { (mg/Las Ca) } \\
(00915)\end{array}$ \\
\hline 430023100115602 & Field blank & $10-06-94$ & 8 & 5.9 & 0.20 & 2.7 & 0.42 \\
\hline 442142098130700 & Laboratory blank & $02-10-94$ & -- & -- & -- & -- & 0.03 \\
\hline 442142098130700 & Laboratory blank & $09-20-96$ & -- & -- & -- & -- & 0.033 \\
\hline 434141100523601 & Field blank & $10-01-96$ & 2 & 7.8 & -- & 1.5 & 0.12 \\
\hline
\end{tabular}

\begin{tabular}{|c|c|c|c|c|c|c|c|c|c|}
\hline $\begin{array}{l}\text { Magne- } \\
\text { sium, } \\
\text { dissolved } \\
\text { (mgh } \\
\text { as Mg) } \\
(00925)\end{array}$ & $\begin{array}{l}\text { Sodium, } \\
\text { dissolved } \\
\text { (mg/L } \\
\text { as Na) } \\
(00930)\end{array}$ & $\begin{array}{l}\text { Potassium, } \\
\text { dissolved } \\
\text { (mg/L } \\
\text { as K) } \\
(00935)\end{array}$ & $\begin{array}{c}\text { Sulfate, } \\
\text { dissolved } \\
\left(\mathrm{mgh}^{2} \text { as }\right. \\
\left.\mathrm{SO}_{4}\right) \\
(00945)\end{array}$ & $\begin{array}{l}\text { Chloride, } \\
\text { dissolved } \\
\text { (mg/L as Cl) } \\
(00940)\end{array}$ & $\begin{array}{c}\text { Fluoride, } \\
\text { dissolved } \\
\text { (mg/L as F) } \\
(00950)\end{array}$ & $\begin{array}{c}\text { Silica, } \\
\text { dissolved } \\
\text { (mgh as } \\
\left.\mathrm{SiO}_{2}\right) \\
(00955)\end{array}$ & $\begin{array}{c}\text { Solids, } \\
\text { residue at } \\
180 \text { deg C, } \\
\text { dissolved } \\
(\mathrm{mg} / \mathrm{L}) \\
(70300)\end{array}$ & $\begin{array}{c}\text { Nitrogen, } \\
\text { ammonia, } \\
\text { dissolved } \\
\text { (mg/L as } \mathrm{N}) \\
(00608)\end{array}$ & $\begin{array}{c}\text { Nitrogen, } \\
\text { nitrite, } \\
\text { dissolved } \\
\text { (mg/L as } \mathrm{N}) \\
(00613)\end{array}$ \\
\hline 0.02 & $<0.20$ & $<0.10$ & 0.70 & $<0.10$ & $<0.10$ & $<0.10$ & 5 & 0.005 & 0.001 \\
\hline 0.004 & 0.027 & -- & -- & -- & -- & -- & -- & $<0.002$ & 0.001 \\
\hline 0.005 & 0.031 & -- & -- & -- & -- & -- & -- & -- & -. \\
\hline 0.02 & $<0.20$ & $<0.10$ & $<0.10$ & $<0.10$ & $<0.10$ & $<0.01$ & $<1$ & $<0.015$ & $<0.010$ \\
\hline
\end{tabular}

\begin{tabular}{|c|c|c|c|c|c|c|c|c|}
\hline $\begin{array}{c}\text { Nitrogen, } \\
\mathrm{NO}_{2}+\mathrm{NO}_{3} \\
\text { dissolved } \\
\text { (mg/L as N) } \\
(00631)\end{array}$ & $\begin{array}{l}\text { Phosphorus, } \\
\text { ortho, } \\
\text { dissolved } \\
\text { (mg/L as P) } \\
\text { (00671) }\end{array}$ & $\begin{array}{c}\text { Arsenic, } \\
\text { dissolved } \\
(\mu \mathrm{g} / \mathrm{L} \text { as As) } \\
(01000)\end{array}$ & $\begin{array}{c}\text { Boron, } \\
\text { dissolved } \\
(\mu g / L \text { as B) } \\
(01020)\end{array}$ & $\begin{array}{c}\text { Iron, } \\
\text { dissolved } \\
(\mu g / L \text { as Fe }) \\
(01046)\end{array}$ & $\begin{array}{l}\text { Manganese, } \\
\text { dissolved } \\
(\mu g / L \text { as Mn) } \\
(01056)\end{array}$ & $\begin{array}{c}\text { Selenium, } \\
\text { dissolved } \\
(\mu g / L \text { as Se) } \\
(01145)\end{array}$ & $\begin{array}{c}\text { Alpha radio, } \\
\text { dissolved } \\
\text { as Th-230 } \\
\text { (pcill) } \\
(\mathbf{0 4 1 2 6 )}\end{array}$ & $\begin{array}{c}\text { Gross beta, } \\
\text { dissolved } \\
\text { (pci/L as } \\
\text { Cs-137) } \\
(03515)\end{array}$ \\
\hline 0.005 & 0.024 & $<1$ & $<10$ & 5 & 9 & $<1$ & -- & -- \\
\hline$<0.005$ & $<0.001$ & -- & $<20$ & 4.8 & $<0.10$ & -- & -- & -- \\
\hline-- & -- & -. & 4.1 & $<3.0$ & 2.9 & -- & -- & -. \\
\hline$<0.050$ & $<0.010$ & $<1$ & 9.7 & 3.0 & $<1.0$ & $<1$ & $<3.0$ & $<4.0$ \\
\hline
\end{tabular}


Table 19. Chemical analyses of field duplicates for observation well water samples

$[\mu \mathrm{S} / \mathrm{cm}$, microsiemens per centimeter; mg/L, milligrams per liter; $\mu \mathrm{g} / \mathrm{L}$, micrograms per liter; pci/L, picocuries per liter; deg $\mathrm{C}$, degrees Celsius; IT, incremental titration; NTU, nephelometric turbidity units; <, less than; --, no data]

\begin{tabular}{|c|c|c|c|c|c|c|}
\hline Station number & Local number & Sample type & Aquifer & Date & $\begin{array}{c}\text { Specific } \\
\text { conduct- } \\
\text { ance, } \\
\text { field } \\
(\mu S / \mathrm{cm}) \\
(00095)\end{array}$ & $\begin{array}{c}\text { Alkalinity, } \\
\text { dissolved, } \\
\text { IT, field } \\
\left(\mathrm{mg}^{\prime} \mathrm{L} \text { as }\right. \\
\left.\mathrm{CaCO}_{3}\right) \\
(39086)\end{array}$ \\
\hline \multirow[t]{2}{*}{431159100412102} & $37 \mathrm{~N} 28 \mathrm{~W} 7 \mathrm{BBBC} 2$ & Sample & Arikaree & $09-21-94$ & 355 & 178 \\
\hline & & Duplicate & -- & $09-21-94$ & $1_{373}$ & ${ }^{\mathrm{I}} 183$ \\
\hline \multirow[t]{2}{*}{431949100584601} & $39 \mathrm{~N} 31 \mathrm{~W} 27 \mathrm{BBBB}$ & Sample & White River & $10-01-96$ & 1,670 & 428 \\
\hline & & Duplicate & -- & $10-01-96$ & ${ }_{1}^{1,690}$ & ${ }^{1} 413$ \\
\hline \multirow[t]{2}{*}{432746100172201} & $40 \mathrm{~N} 25 \mathrm{~W} 4 \mathrm{CCDD}$ & Sample & Alluvial & $09-30-96$ & ${ }^{1} 1,830$ & 257 \\
\hline & & Duplicate & -- & 09-30-96 & ${ }^{1} 1,810$ & $1_{251}$ \\
\hline
\end{tabular}

\begin{tabular}{|c|c|c|c|c|c|c|c|}
\hline $\begin{array}{c}\text { Calcium, } \\
\text { dissolved } \\
\text { (mg/L as Ca) } \\
(00915)\end{array}$ & $\begin{array}{l}\text { Magnesium, } \\
\text { dissolved } \\
\text { (mg/L as Mg) } \\
\text { (00925) }\end{array}$ & $\begin{array}{c}\text { Sodium, } \\
\text { dissolved } \\
\text { (mg/L as Na) } \\
(00930)\end{array}$ & $\begin{array}{l}\text { Potassium, } \\
\text { dissolved } \\
(\mathrm{mg} / \mathrm{L} \text { as K) } \\
(00935)\end{array}$ & $\begin{array}{c}\text { Sulfate, } \\
\text { dissolved } \\
\left(\mathrm{mg} / \mathrm{h} \text { as } \mathrm{SO}_{4}\right) \\
(00945)\end{array}$ & $\begin{array}{c}\text { Chloride, } \\
\text { dissolved } \\
\text { (mg/L as Cl) } \\
(00940)\end{array}$ & $\begin{array}{c}\text { Fluoride, } \\
\text { dissolved } \\
\text { (mg/L as F) } \\
(00950)\end{array}$ & $\begin{array}{c}\text { Silica, } \\
\text { dissolved } \\
(\mathrm{mg} / \mathrm{L} \text { as Sio } \\
(00955)\end{array}$ \\
\hline 37 & 7.2 & 28 & 9.6 & 9.3 & 1.6 & 0.40 & 62 \\
\hline 38 & 7.3 & 28 & 9.4 & 9.3 & 1.7 & 0.40 & 62 \\
\hline 10 & 0.60 & 350 & 11 & 0.10 & 300 & 1.4 & 16 \\
\hline 9.7 & 0.57 & 340 & 11 & 0.20 & 300 & 1.4 & 16 \\
\hline 270 & 54 & 72 & 16 & 810 & 22 & 0.20 & 37 \\
\hline 270 & 55 & 73 & 17 & 810 & 22 & 0.20 & 36 \\
\hline
\end{tabular}

\begin{tabular}{|c|c|c|c|c|c|c|}
\hline $\begin{array}{c}\text { Solids, } \\
\text { residue at } 180 \\
\text { deg C, } \\
\text { dissolved } \\
\text { (mg/L) } \\
(70300)\end{array}$ & $\begin{array}{c}\text { Nitrogen, } \\
\text { ammonia, } \\
\text { dissolved } \\
\text { (mg/L as } N) \\
(00608)\end{array}$ & $\begin{array}{l}\text { Nitrogen, nitrite, } \\
\text { dissolved } \\
\text { (mg/L as N) } \\
(00613)\end{array}$ & $\begin{array}{c}\text { Nitrogen, } \\
\mathrm{NO}_{2}+\mathrm{NO}_{3} \\
\text { dissolved } \\
(\mathrm{mg} / \mathrm{L} \text { as } \mathrm{N}) \\
(00631)\end{array}$ & $\begin{array}{c}\text { Phosphorus, } \\
\text { ortho, } \\
\text { dissolved } \\
\text { (mg/L as P) } \\
\text { (00671) }\end{array}$ & $\begin{array}{c}\text { Arsenic, } \\
\text { dissolved } \\
(\mu \mathrm{g} / \mathrm{L} \text { as As) } \\
(01000)\end{array}$ & $\begin{array}{c}\text { Boron, } \\
\text { dissolved } \\
(\mu g / L \text { as B) } \\
(01020)\end{array}$ \\
\hline 260 & $<0.002$ & $<0.001$ & 1.50 & 0.360 & 7 & 30 \\
\hline 269 & $<0.002$ & $<0.001$ & 1.50 & 0.352 & 6 & 40 \\
\hline 952 & 0.450 & $<0.010$ & $<0.050$ & 0.030 & 1 & 2,120 \\
\hline 948 & 0.440 & $<0.010$ & $<0.050$ & 0.030 & 1 & 2,030 \\
\hline 1,540 & 0.080 & $<0.010$ & 0.700 & 0.070 & 3 & 191 \\
\hline 1,550 & 0.080 & $<0.010$ & 0.660 & 0.070 & 4 & 185 \\
\hline
\end{tabular}


Table 19. Chemical analyses of field duplicates for observation well water samples-Continued

\begin{tabular}{|c|c|c|c|c|c|c|}
\hline $\begin{array}{c}\text { Iron, } \\
\text { dissolved } \\
(\mu g / \text { as } F e) \\
(01046)\end{array}$ & $\begin{array}{c}\text { Manganese, } \\
\text { dissolved } \\
(\mu g / \text { as Mn) } \\
(01056)\end{array}$ & $\begin{array}{c}\text { Selenium, } \\
\text { dissolved } \\
(\mu g / \text { as } \mathrm{Se}) \\
(01145)\end{array}$ & $\begin{array}{c}\text { Gross alpha, } \\
\text { dissolved } \\
\text { ( } \mu \mathrm{g} / \mathrm{L} \text { as } \\
\text { natural } \\
\text { uranium) } \\
(80030)\end{array}$ & $\begin{array}{l}\text { Alpha radio, } \\
\text { dissolved } \\
\text { as Th-230 } \\
\text { (pci/L) } \\
(04126)\end{array}$ & $\begin{array}{c}\text { Gross beta, } \\
\text { dissolved } \\
\text { (pci/L as } \\
\text { Cs-137) } \\
(03515)\end{array}$ & $\begin{array}{c}\text { Gross beta, } \\
\text { dissolved } \\
\text { (pci/l as } \\
\text { Sr/Yt-90) } \\
(80050)\end{array}$ \\
\hline$<3$ & $<1$ & $<1$ & 5.6 & 3.9 & 9.9 & 7.3 \\
\hline 18 & 19 & $<1$ & -- & 6.1 & 26 & -- \\
\hline 17 & 18 & $<1$ & -- & 4.9 & 30 & -- \\
\hline 230 & 250 & 11 & -- & 3.9 & 22 & -- \\
\hline 50 & 240 & 14 & -. & 4.7 & 25 & -- \\
\hline
\end{tabular}

\footnotetext{
${ }^{1}$ Indicates laboratory value
} 
Table 20. Precision of chemical analyses of field duplicates for observation well water samples $[\mu \mathrm{S} / \mathrm{cm}$, microsiemens per centimeter; $\mathrm{mg} / \mathrm{L}$, milligrams per liter; $\mu \mathrm{g} / \mathrm{L}$, micrograms per liter; $\mathrm{pci} / \mathrm{L}$, picocuries per liter]

\begin{tabular}{|c|c|c|c|}
\hline Constituent and reporting unit & $\begin{array}{c}\text { Number of duplicate } \\
\text { pairs }\end{array}$ & $\begin{array}{l}\text { Standard deviation } \\
\text { in units }\end{array}$ & $\begin{array}{c}\text { Relative standard deviation, } \\
\text { in percent }\end{array}$ \\
\hline Specific conductance, $\mu \mathrm{S} / \mathrm{cm}$ & 3 & 13.7 & 1.1 \\
\hline Alkalinity, $\mathrm{mg} / \mathrm{L}$ as calcium carbonate & 3 & 6.9 & 2.4 \\
\hline Calcium, mg/L & 3 & 0.43 & 0.41 \\
\hline Magnesium, mg/L & 3 & 0.41 & 2.0 \\
\hline Sodium, $\mathrm{mg} / \mathrm{L}$ & 3 & 4.1 & 2.8 \\
\hline Potassium, mg/L & 3 & 0.42 & 3.4 \\
\hline Sulfate, $\mathrm{mg} / \mathrm{L}$ & 3 & 0.04 & 0.02 \\
\hline Chloride, $\mathrm{mg} / \mathrm{L}$ & 3 & 0.04 & 0.04 \\
\hline Fluoride, $\mathrm{mg} / \mathrm{L}$ & 3 & 0.0 & 0.0 \\
\hline Silica, mg/L & 3 & 0.41 & 1.1 \\
\hline Solids, mg/L & 3 & 5.7 & 0.62 \\
\hline Ammonia, $\mathrm{mg} / \mathrm{L}$ as $\mathrm{N}$ & 3 & 0.004 & 2.3 \\
\hline Nitrite, $\mathrm{mg} / \mathrm{L}$ as $\mathrm{N}$ & 3 & 0.0 & 0.0 \\
\hline Nitrite + Nitrate, $\mathrm{mg} / \mathrm{L}$ as $\mathrm{N}$ & 3 & 0.02 & 2.7 \\
\hline Phosphorus, ortho, $\mathrm{mg} / \mathrm{L}$ as $\mathrm{P}$ & 3 & 0.003 & 2.0 \\
\hline Arsenic, $\mu \mathrm{g} / \mathrm{L}$ & 3 & 0.58 & 16 \\
\hline Boron, $\mu \mathrm{g} / \mathrm{L}$ & 3 & 37 & 4.8 \\
\hline Iron, $\mu \mathrm{g} / \mathrm{L}$ & 3 & 73 & 140 \\
\hline Manganese, $\mu \mathrm{g} / \mathrm{L}$ & 3 & 4.1 & 4.7 \\
\hline Selenium, $\mu \mathrm{g} / \mathrm{L}$ & 3 & 1.2 & 27 \\
\hline Gross alpha, pci/L as natural uranium & 1 & 0.99 & 20 \\
\hline Alpha, pci/L as $\mathrm{Th}-230$ & 3 & 0.79 & 18 \\
\hline Gross beta, pci/L as Cs- 137 & 3 & 2.1 & 10 \\
\hline Gross beta, $\mathrm{pci} / \mathrm{L}$ as $\mathrm{Sr} / \mathrm{Yt}-90$ & 1 & 0.28 & 3.9 \\
\hline
\end{tabular}


